\title{
Platelet-derived SDF-1 primes the pulmonary capillary vascular niche to drive lung alveolar regeneration
}

\author{
Shahin Rafii ${ }^{1,2,3,4}$, Zhongwei Cao ${ }^{1,2,7}$, Raphael Lis ${ }^{1,2,4,7}$, Ilias I. Siempos ${ }^{2,5,7}$, Deebly Chavez ${ }^{1,2,3}$, Koji Shido ${ }^{1,2}$, \\ Sina Y. Rabbany ${ }^{1,2,6}$ and Bi-Sen Ding ${ }^{1,3,8}$
}

The lung alveoli regenerate after surgical removal of the left lobe by pneumonectomy (PNX). How this alveolar regrowth/regeneration is initiated remains unknown. We found that platelets trigger lung regeneration by supplying stromal-cell-derived factor-1 (SDF-1, also known as CXCL12). After PNX, activated platelets stimulate SDF-1 receptors CXCR4 and CXCR7 on pulmonary capillary endothelial cells (PCECs) to deploy the angiocrine membrane-type metalloproteinase MMP14, stimulating alveolar epithelial cell (AEC) expansion and neo-alveolarization. In mice lacking platelets or platelet Sdf1, PNX-induced alveologenesis was diminished. Reciprocally, infusion of Sdf1+/+ but not Sdf1-deficient platelets rescued lung regeneration in platelet-depleted mice. Endothelial-specific ablation of $\mathrm{Cxcr} 4$ and $\mathrm{Cxcr} 7$ in adult mice similarly impeded lung regeneration. Notably, mice with endothelial-specific Mmp14 deletion exhibited impaired expansion of AECs but not PCECs after PNX, which was not rescued by platelet infusion. Therefore, platelets prime PCECs to initiate lung regeneration, extending beyond their haemostatic contribution. Therapeutic targeting of this haemo-vascular niche could enable regenerative therapy for lung diseases.

In mammals, surgical removal of the left lung by pneumonectomy (PNX) leads to restoration of mass and gas exchange function in the residual lung alveoli ${ }^{1-8}$. This process of compensatory lung regrowth/regeneration results from formation of new functional alveolar units. PNX-induced neo-alveolarization is driven by expansion of several cell types, which include alveolar epithelial cells (AECs; refs 9-13), endothelial cells ${ }^{1,14-19}$ and mesenchymal cells ${ }^{3}$. However, it remains unknown how the loss of the lung lobe triggers the alveolar regeneration in the remaining lungs.

Pulmonary capillary endothelial cells (PCECs) lining the lung microvasculature form a specialized vascular niche to modulate the function of alveolar progenitor cells ${ }^{11-18}$. Following PNX, upregulation of the membrane-type metalloprotease MMP14 (refs 20,21) in PCECs (ref. 1) leads to release of cryptic epidermal growth factor (EGF)like ligands to stimulate the propagation of alveolar progenitor cells such as type II alveolar epithelial cells (AEC2s; refs 9,10), initiating the regenerative alveolarization. Blockage of MMP14 activity by neutralizing antibody impeded the expansion of AECs but not PCECs, impairing neo-alveolarization and restoration of alveolar function. However, the alveologenic function of endothelial-derived MMP14 after PNX remains to be further demonstrated ${ }^{22,23}$. Moreover, the cellular and molecular basis whereby MMP14 is specifically induced in the pulmonary vascular niche $e^{1,24,25}$ by PNX is not defined.

Platelets are anuclear cells that constantly transit through the lung blood vessels ${ }^{26,27}$. Activated platelets not only prevent haemorrhage by forming clots, but also promote organ repair through 'inside-out' mobilization of trophogens and adhesion molecules ${ }^{28-38}$. Enriched with peptide and lipid mediators in granules ${ }^{39,40}$, platelets orchestrate multiple pathophysiological processes jointly with endothelial cells (ECs; refs 41,42). In response to stress, platelets are activated and adhere to the vascular bed of injured organs ${ }^{43-46}$. The intimate relationship between platelets and the lung vasculature ${ }^{26,33}$ set forth the hypothesis that resection of the left lung recruits circulating platelets to prime the pulmonary vascular niche, driving alveolar regeneration.

\section{RESULTS}

After PNX, recruited CD41+ ${ }^{+}$platelets are essential in initiating lung alveolar regeneration

To test the pulmonary recruitment of platelets by PNX, we carried out flow cytometry analysis of platelet-specific marker CD41 (also known as ITGA2B) in total cells retrieved from the remaining lobes of pneumonectomized lungs. PNX caused significant enrichment of

${ }^{1}$ Ansary Stem Cell Institute, Weill Cornell Medical College, New York, New York 10065, USA. ${ }^{2}$ Department of Medicine, Weill Cornell Medical College, New York, New York 10065, USA. ${ }^{3}$ Department of Genetic Medicine, Weill Cornell Medical College, New York, New York 10065, USA. ${ }^{4}$ Department of Reproductive Medicine, Weill Cornell Medical College, New York, New York 10065, USA. ${ }^{5}$ First Department of Critical Care Medicine and Pulmonary Services, Evangelismos Hospital, University of Athens Medical School, Athens 10675, Greece. ${ }^{6}$ Bioengineering Program, Hofstra University, Hempstead, New York 11549, USA. ${ }^{7}$ These authors contributed equally to this work.

${ }^{8}$ Correspondence should be addressed to B-S.D. (e-mail: bid2004@med.cornell.edu)

Received 7 April 2014; accepted 16 December 2014; published online 26 January 2015; DOI: 10.1038/ncb3096 
a

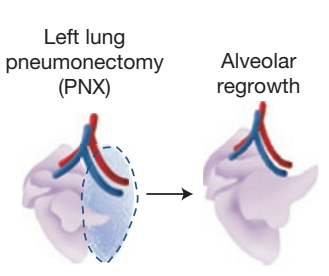

b

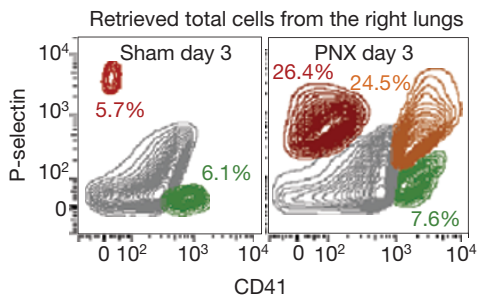

c

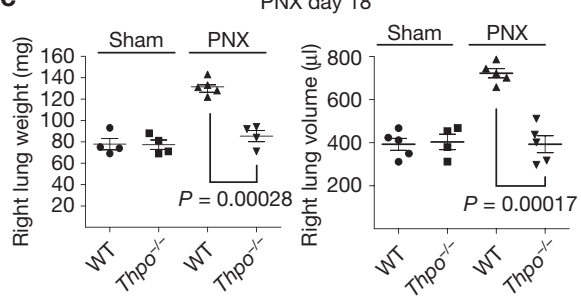

d

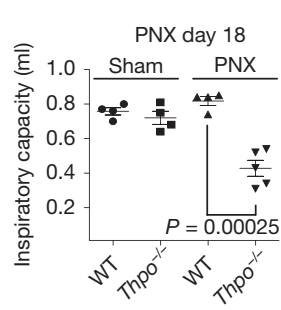

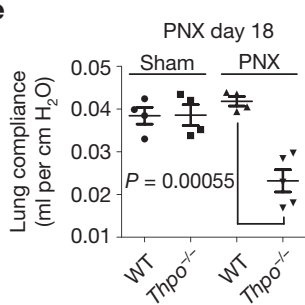

f
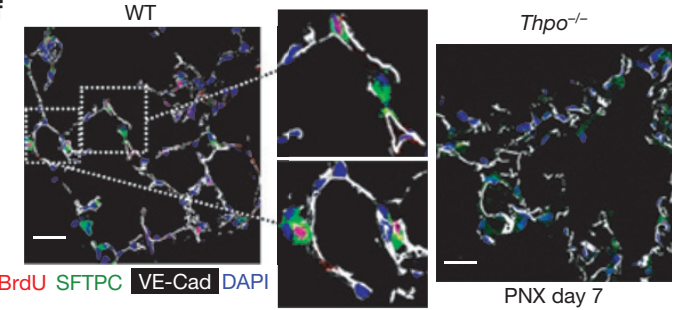

g

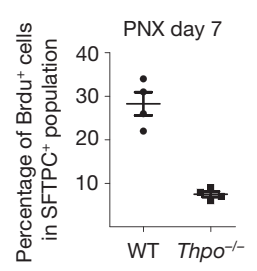

h

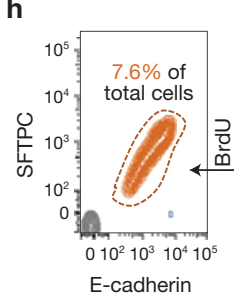

Retrieved lung total cells from

pneumonectomized mice (PNX day 7)

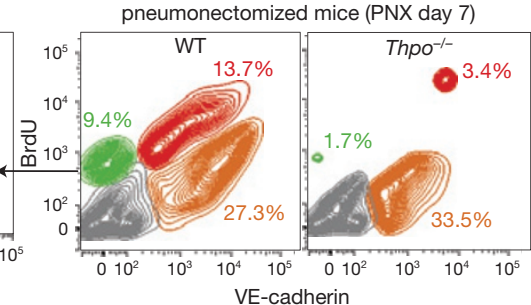

i

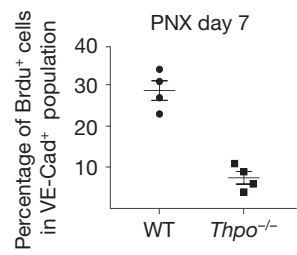

j

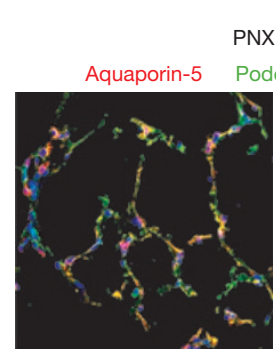

WT

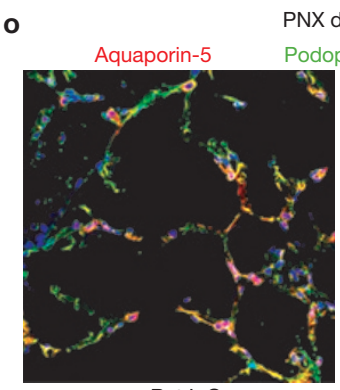

Rat IgG
PNX day 18

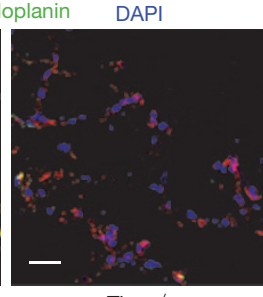

Thpo $^{-/-}$

NX day 18
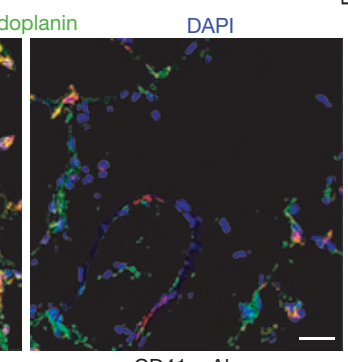

CD41 mAb

k
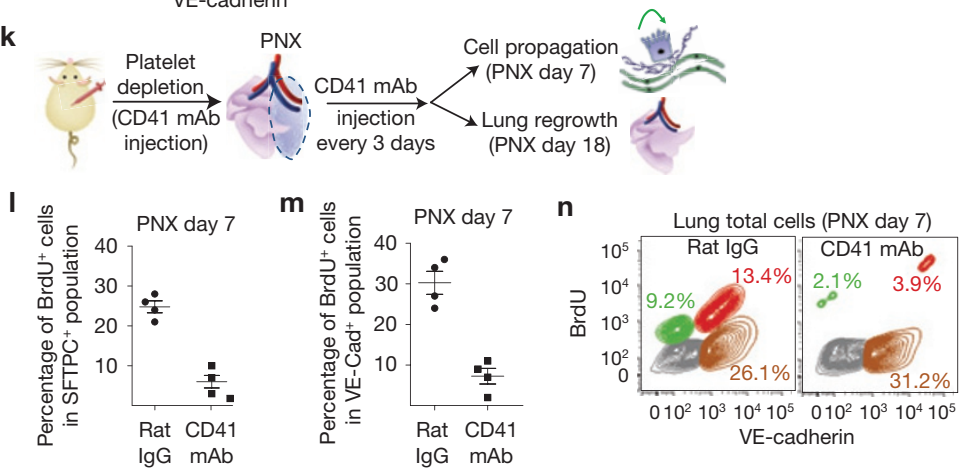

p
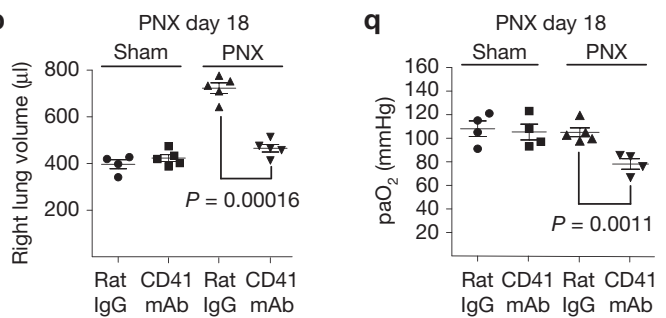

Figure 1 After surgical removal of the left lung lobe by PNX, platelets are essential for the regrowth/regeneration of the remaining right lung. (a,b) After PNX (a), surface expression of activation marker P-selectin on $\mathrm{CD} 41^{+}$ platelets was examined by flow cytometry (b). Sham-operated mice underwent thoracotomy without lung resection. $\mathrm{CD}_{1} 1^{+} \mathrm{P}$-selectin ${ }^{+}$activated platelets are denoted in yellow (b). (c) Weight and volume of the right lungs in WT and TPO null ( $\mathrm{ThpO}^{-/-}$) mice lacking circulating platelets. Left panel: $n=4$ mice (both sham groups), $n=5$ mice (WT with PNX) and $n=4$ mice ( Thpo $^{-1-}$ with PNX). Right panel: $n=5$ mice (WT with sham), $n=4$ animals ( Thpo $^{-1-}$ with sham) and $n=5$ animals for both PNX groups. (d,e) Inspiratory capacity (d) and lung compliance (e) in WT and Thpo-/- mice. $n=4$ mice (both sham groups), $n=4$ mice (WT with PNX) and $n=5$ mice (pneumonectomized Thpo-/- group). $(\mathbf{f}-\mathbf{j})$ BrdU incorporation in SFTPC ${ }^{+}$AEC2s and VE-cadherin ${ }^{+}$PCECs was tested in mice by immunostaining and flow cytometry, respectively. DAPI, 4',6-diamidino-2-phenylindole. Characterization of the lungs of the sham mice is shown in Supplementary Fig. 1. (g,i) $n=4$ mice in all tested groups; $P=0.00027$ (g) and 0.0003 (i) between two groups. Scale bar, $50 \mu \mathrm{m}$. (j) Distribution of $\mathrm{AEC} 1 \mathrm{~s}$ expressing aquaporin 5 and podoplanin in mice after PNX. (k-q) Lung regrowth in thromobocytopenic mice after PNX. Mice were injected with anti-CD41 mAb to deplete platelets (k). Propagation of AEC2s (I) and PCECs $(\mathbf{m}, \mathbf{n})$, localization of AEC1s (o), right lung volume (p) and arterial oxygen level $(\mathbf{q})$ were measured in mice. $(\mathbf{I}, \mathbf{m}) n=4$ mice in all groups; $P=0.00013(\mathbf{I})$ and $0.00054(\mathbf{m})$ between two groups. (p) $n=3$ mice (IgG-treated sham), $n=5$ mice (CD41 mAb-injected sham) and $n=5$ mice (both PNX); (q) $n=4$ mice (both sham), $n=5$ mice (PNX with IgG) and $n=4$ mice (PNX with CD41 mAb). Scale bar, $50 \mu \mathrm{m}$. The error bar indicates s.e.m., and the line represents the mean for $\mathbf{c}-\mathbf{e}, \mathbf{g}, \mathbf{i}, \mathbf{I}, \mathbf{m}, \mathbf{p}, \mathbf{q}$. The difference between individual groups was compared by unpaired two-tailed $t$-test. 
a

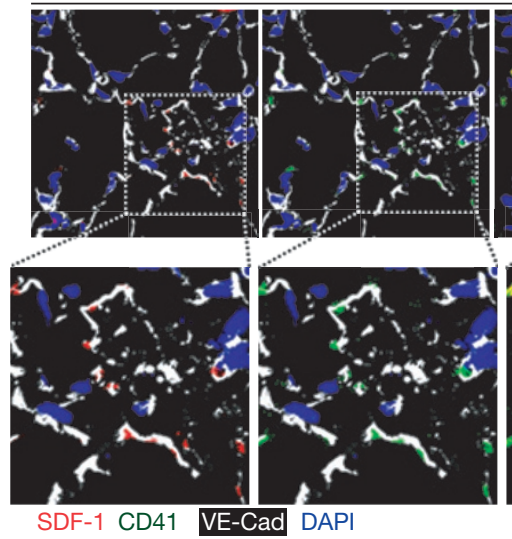

C

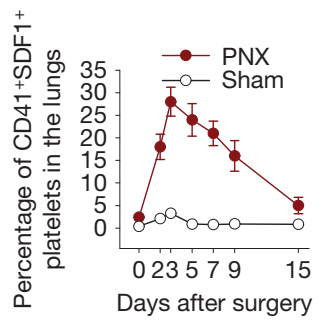

g

Rosa-CreERT2

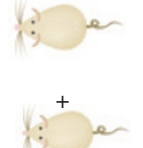

Sdf1 LoxP/LoxP

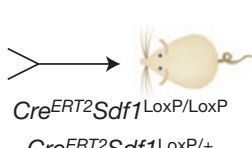

Cre ${ }^{E R T 2 S} d f 1^{\text {LoxP/+ }}$

i

SDF-1 protein in isolated platelets

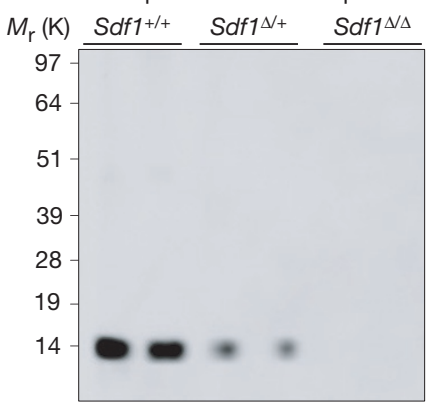

PNX day 3
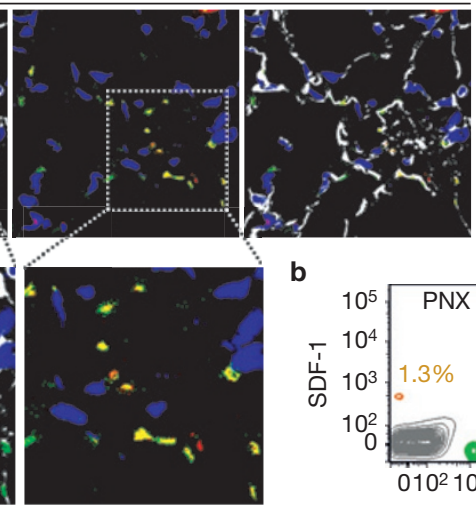

b
Sham day 3

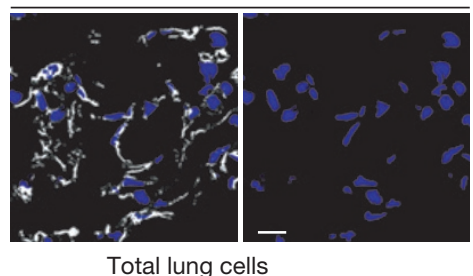

105

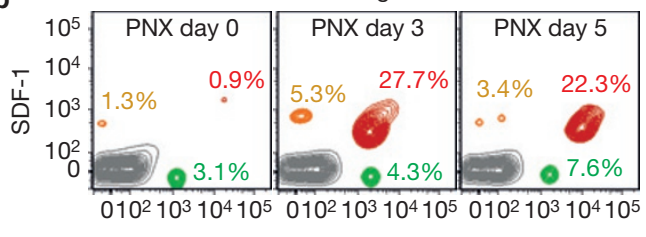

CD41 d

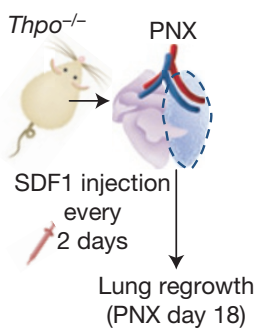

e
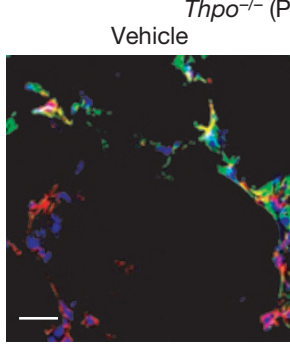

Aquaporin-5
Thpo-/- (PNX day 18)

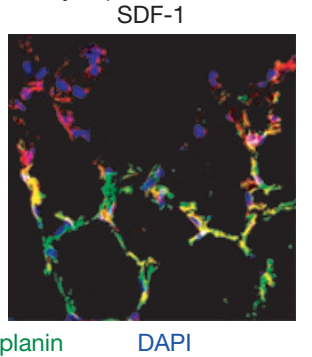

f

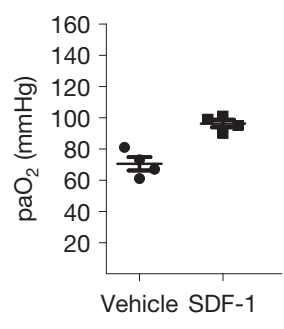

h

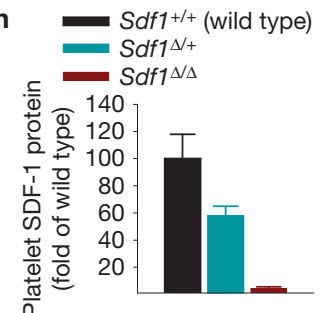

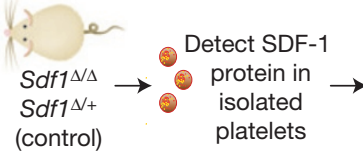
(control) isolated $\begin{array}{ccc}150 \mathrm{mg} \mathrm{kg}^{-1} & \text { Rest } & 150 \mathrm{mg} \mathrm{kg}^{-1} \\ \text { every day } & \text { for } & \text { every day }\end{array}$

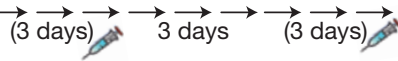

j $\beta$-actin protein in isolated platelets

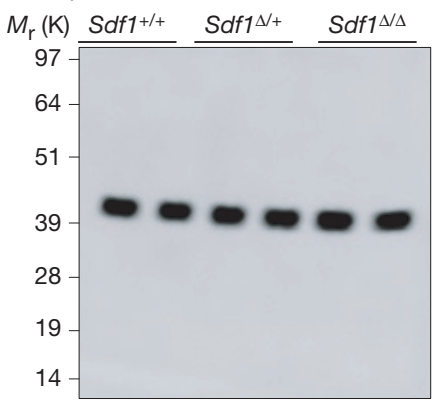

j BrdU SFTPC DAPI

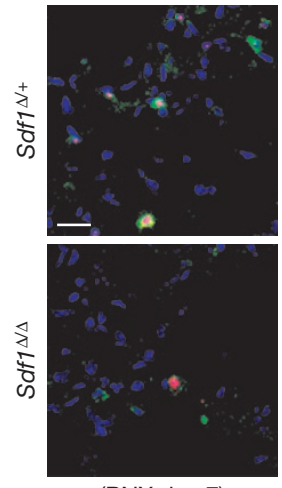

Day 18

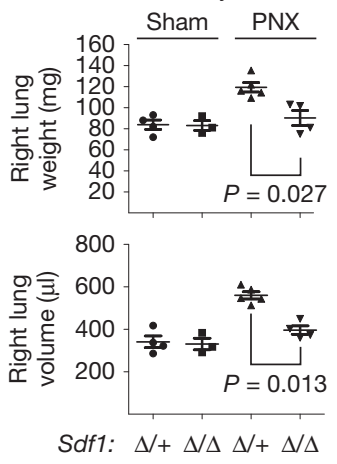

(PNX day 7)

Figure 2 Pulmonary accumulation of platelets carrying SDF-1 (CXCL12) after PNX. (a) Immunostaining of SDF-1, VE-cadherin and CD41 in mouse lung cryosections. CD41 ${ }^{+} \mathrm{SDF}-1^{+}$platelets were associated with VE-cadherin ${ }^{+}$ PCECs after PNX (inset). Scale bar, $50 \mu \mathrm{m}$. (b,c) Flow cytometry analysis of SDF-1+ $1^{+}$platelet recruitment in the remaining lungs after PNX. Red depicts SDF- $1^{+}$CD $41^{+}$platelets, and small amounts of CD41-SDF-1+ cells are presented in yellow. The kinetics of platelet accumulation in the lungs after PNX is assessed (c). $n=6$ animals at each time. (d-f) Mouse alveolar regrowth in $\mathrm{Thpo}^{-1-}$ mice after injection of recombinant SDF-1. Thpo-lmice were treated with SDF-1 injection, and AEC1 distribution (e) and gas exchange function (f) in treated mice was assessed by comparing with the vehicle-injected group. $n=4$ mice in both groups; $P=0.0055$ between two groups; scale bar, $50 \mu \mathrm{m}$. (g-i) Inducible deletion of $S d f 1$ in adult mice $\left(S d f 1^{\Delta / \Delta}\right)$. Mice expressing tamoxifen-responsive Rosa-Cre $e^{\text {RT2 }}$ were crossed with $S d f 1^{\operatorname{lox} / / \mathrm{lox} P}$ mice to generate $S d f 1^{\Delta / \Delta}$ and control $S d f 1^{\Delta /+}$ mice. SDF-1 protein was determined by immunoblot in isolated platelets from mice 30 days after the last injection. The protein level was compared after normalization to $\beta$-actin; a representative immunoblot image is shown (i); each lane indicates an individual animal, and mice were derived from two cohorts for the analysis ( $n=6$ mice in all genotypes). ( $\mathbf{j}, \mathbf{k})$ Proliferation of AEC2s (j) and lung regrowth (k) in Sdf1 $1^{\Delta / \Delta}$ and control mice after PNX. (k) $n=4$ and 3 mice in sham-operated control and $S d f 1^{\Delta / \Delta}$ mice, $n=5$ and 4 mice in control and $S d f 1^{\Delta / \Delta}$ mice that underwent PNX. Scale bar, $50 \mu \mathrm{m}$. The error bar depicts the s.e.m., and the line stands for the mean for $\mathbf{c}, \mathbf{f , h}$ and $\mathbf{k}$. Statistical difference between groups was assessed by an unpaired two-tailed $t$-test. 


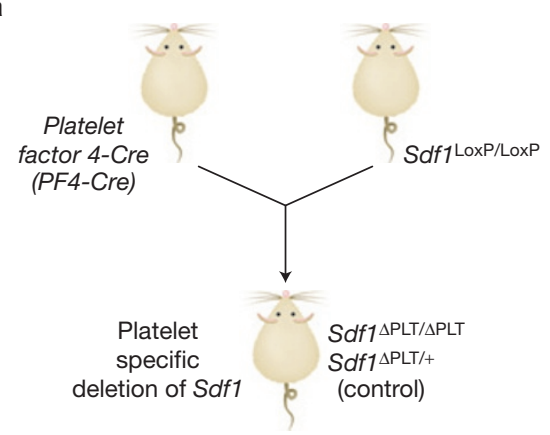

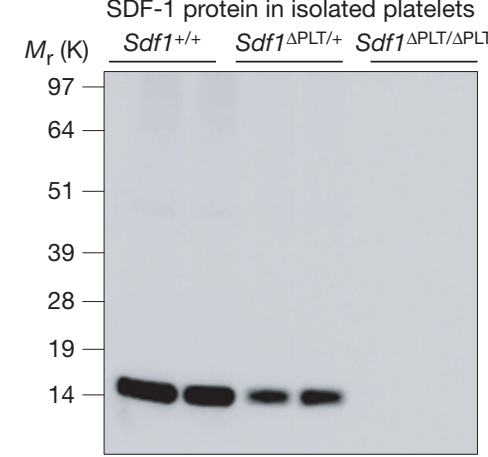

$\beta$-actin protein in isolated platelets
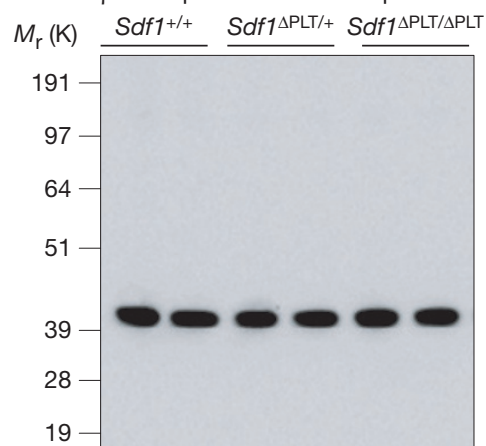

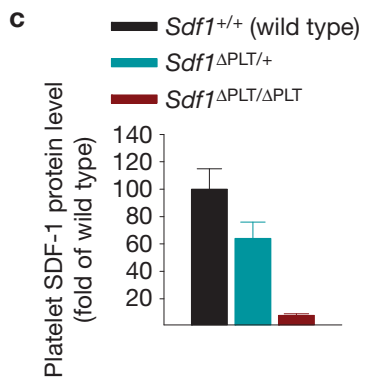

d

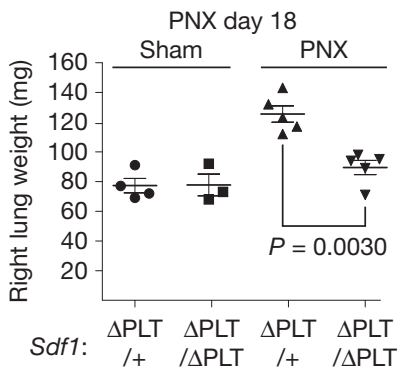

g
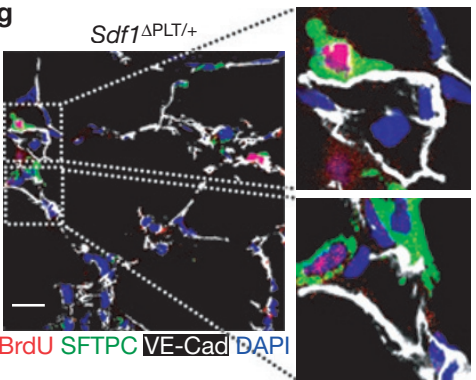

Sdf1 $\triangle \mathrm{PLT} / \Delta \mathrm{PLT}$

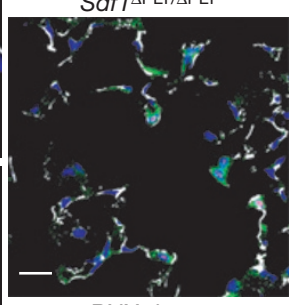

PNX day 7

h

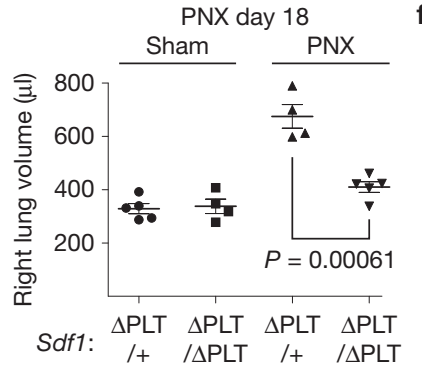

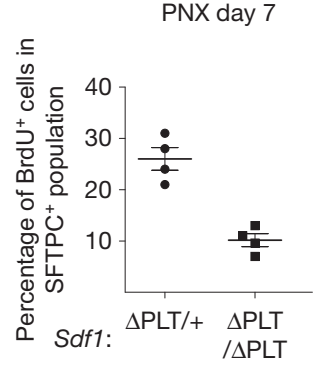

Total lung cells (PNX day 7 )

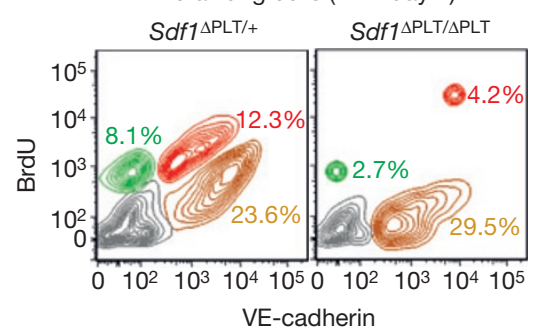

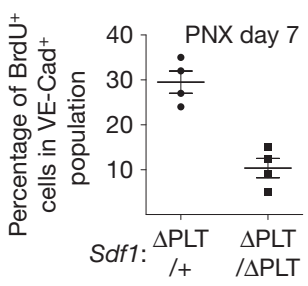

j

Sdf1 $1 \mathrm{PLT} /+$

$S d f 1 \Delta \mathrm{PLT} / \Delta \mathrm{PLT}$

$\mathbf{k}$

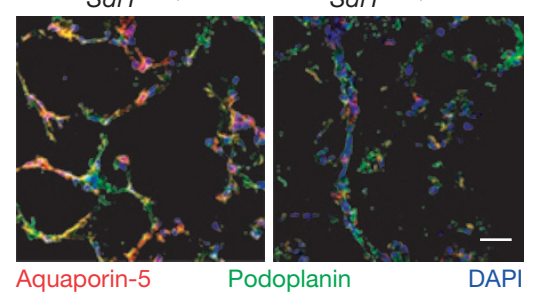

Figure 3 Platelets recruited by PNX derive SDF-1 to elicit alveolar regrowth/regeneration. (a-c) Platelet-specific deletion of $S d f 1$ ( $S d f 1^{\Delta \mathrm{PLT} / \Delta \mathrm{PLT}}$ ) from mice. (a) Mouse line with platelet/platelet progenitor-specific Platelet factor 4 promoter-driven Cre (PF4-Cre) was crossed with $S d f 1^{\text {loxP/loxP }}$ mice to delete $S d f 1$ specifically from platelets. $S d f 1^{\Delta \mathrm{PLT} /+}$ mice harbouring haplodeficiency of Sdf1 in platelets served as control. (b,c) SDF-1 protein in platelets was analysed by immunoblot to determine $S d f 1$ deletion efficiency. In the blot image in $\mathbf{b}$, each lane indicates platelets from an individual animal. Mice from two cohorts were tested in each group $(n=6$ mice per group). (d,e) Right lung weight (d) and volume (e) in $S d f 1^{\triangle \mathrm{PLT} / \Delta \mathrm{PLT}}$ and control mice after PNX. (d) $n=4$ and 3 control and $S d f 1^{\triangle P L T / \triangle P L T}$ mice (sham); $n=5$ control and $S d f 1^{\Delta \mathrm{PLT} / \triangle \mathrm{PLT}}$ mice (PNX). (e) $n=5$ and 4 control

$\mathrm{CD}_{4} 1^{+}$platelets in the lungs after PNX (Fig. 1a,b). In control mice, whereby thoracotomy was carried out without lung resection (sham), there was negligible deposition of platelets. Surface expression of Pselectin, a platelet activation marker, was detected in the majority of $\mathrm{CD}_{4} 1^{+}$platelets in the pneumonectomized lungs (Fig. 1b). Thus, and $S d f 1^{\triangle P L T / \triangle P L T}$ (sham) mice; $n=4$ and 5 pneumonectomized control and Sdf $1^{\triangle \mathrm{PLT} / \triangle \mathrm{PLT}}$ mouse groups. Scale bar, $50 \mu \mathrm{m}$. (f-i) Proliferation of AEC2s and PCECs in Sdf1 $1^{\triangle P L T / \triangle P L T}$ and control mice after PNX. Expansion of AEC2s and PCECs at day 7 after PNX was determined by immunostaining $(\mathbf{f}, \mathbf{g})$ and flow cytometry $(\mathbf{h}, \mathbf{i})$, respectively; $n=4$ mice per group in $\mathbf{f}, \mathbf{i}$; $P=0.001$ (f) and 0.0011 (i) between the two genotypes. Scale bar, $50 \mu \mathrm{m}$. (j) Restoration of $\mathrm{AECls}$ in $S d f 1^{\triangle \mathrm{PLT} / \triangle \mathrm{PLT}}$ and control mice after PNX. Scale bar, $50 \mu \mathrm{m}$. (k) After PNX, activated platelets adhere to PCECs and supply SDF-1 to drive regenerative alveolarization. The error bar denotes the s.e.m., and the line describes the mean for c-e,i. An unpaired twotailed $t$-test was used to determine the statistical difference between individual groups.

activated platelets are recruited to the residual right lungs after surgical removal of the left lung lobe.

To assess the contribution of platelets to alveolar regrowth, we used Thrombopoietin-deficient $\left(\mathrm{Thpo}^{-/-}\right)$mice in which the circulating platelet number is $5 \%$ of that of wild-type (WT) 


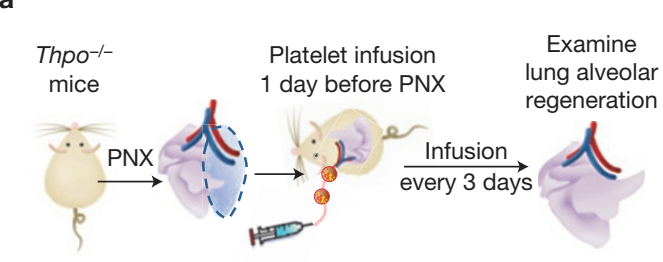

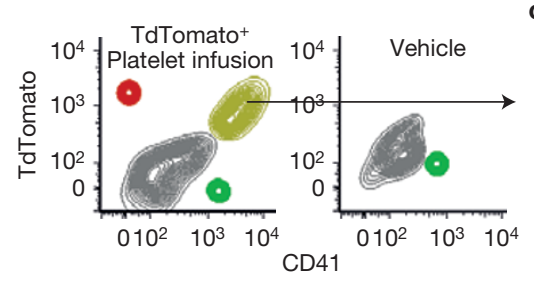

c TdTomato $\mathrm{CD} 41$ VE-Cad Nuclei

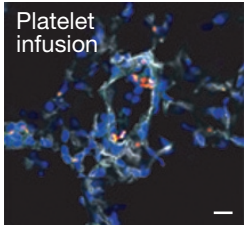

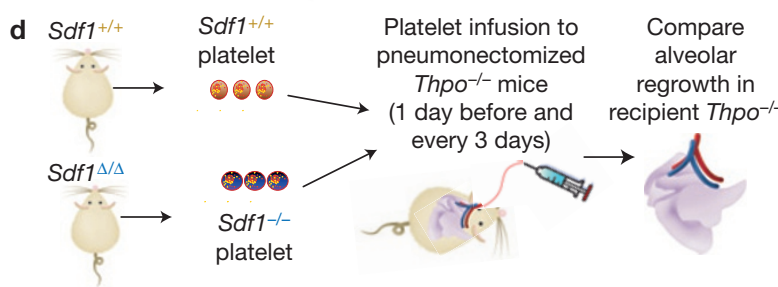
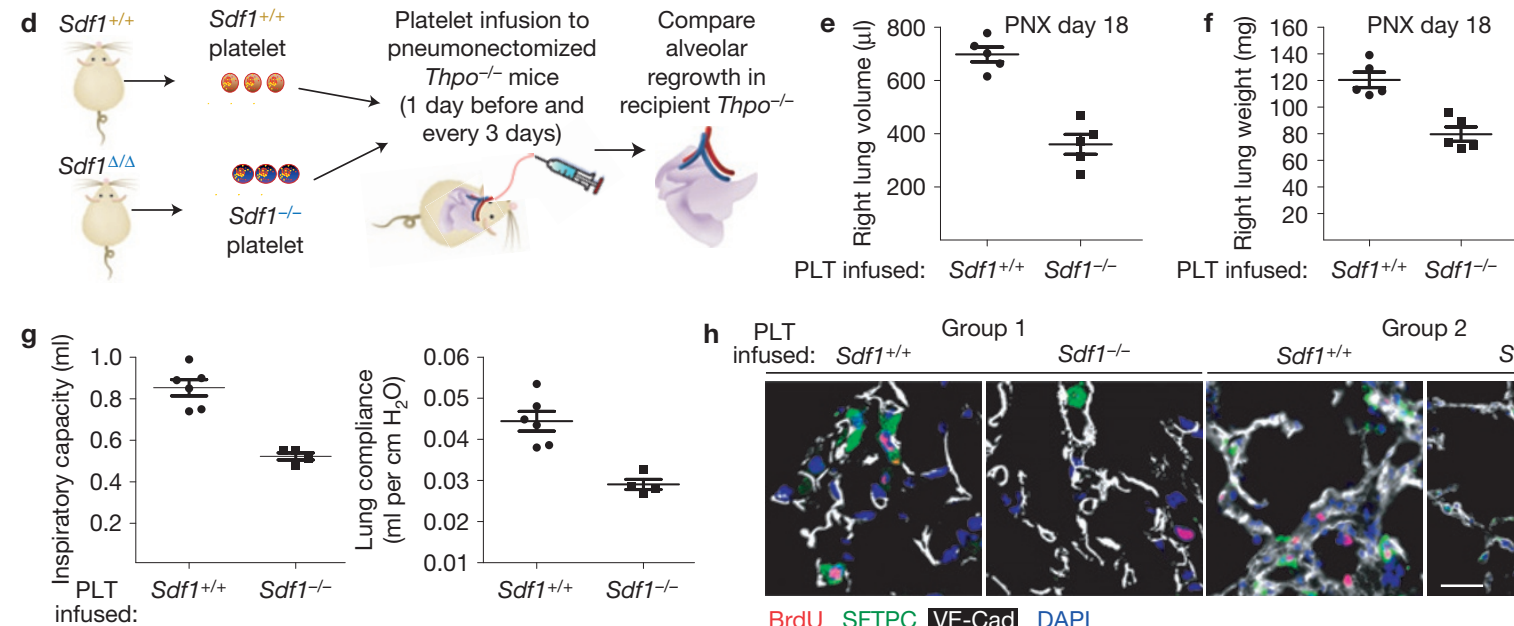

h $\begin{gathered}\text { PLT } \\ \text { infused: }\end{gathered}$ Sdf1+/+ $\quad$ Group $1 \quad S d f 1^{-/-}$

PLT infused: Sdf1+/+ Sdf1-/-

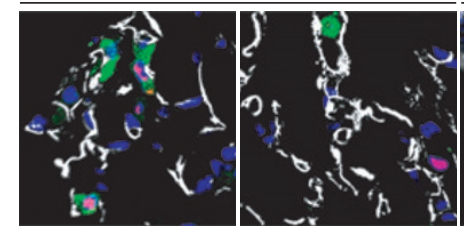

BrdU SFTPC VE-Cad DAPI
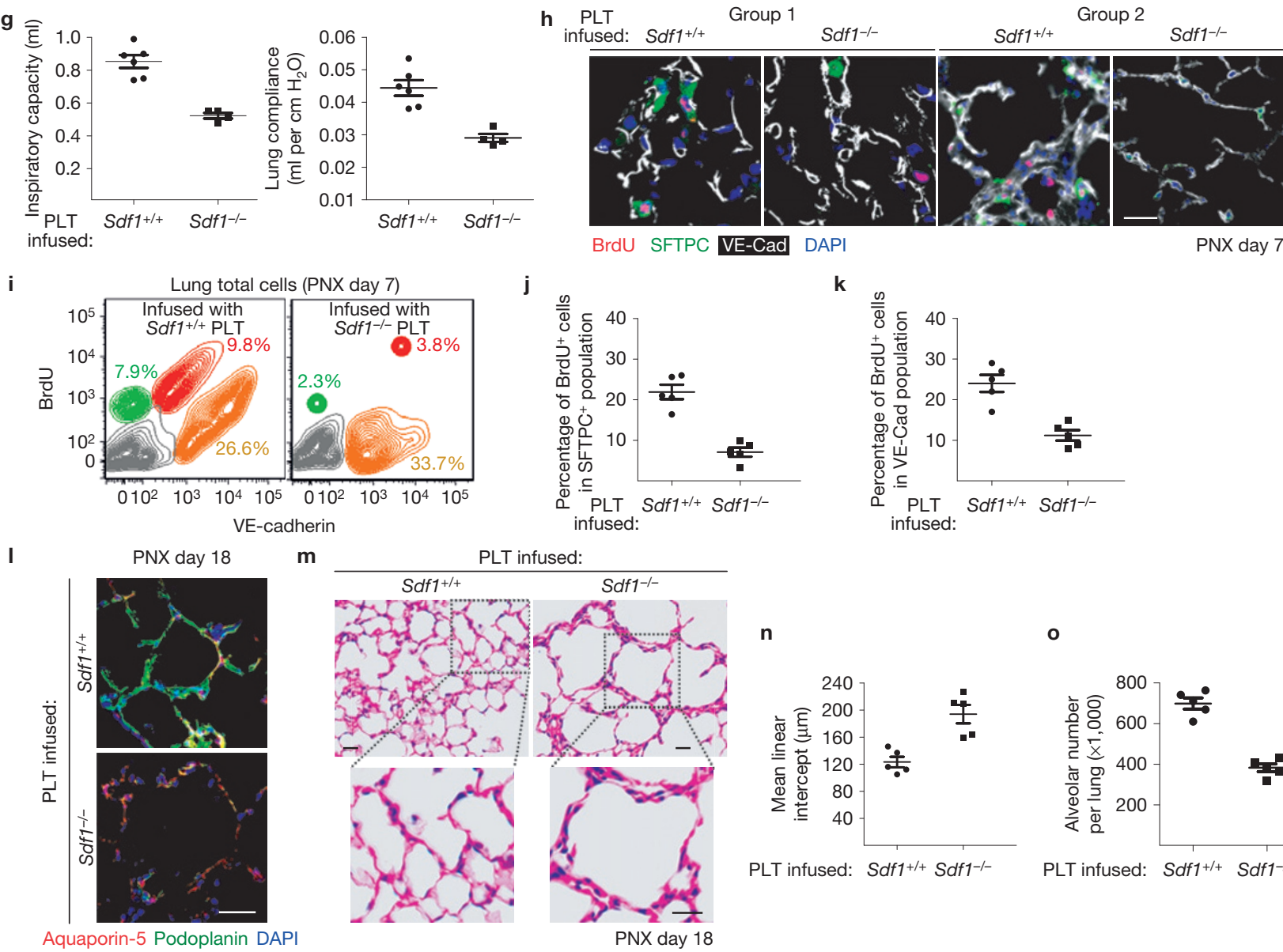

n

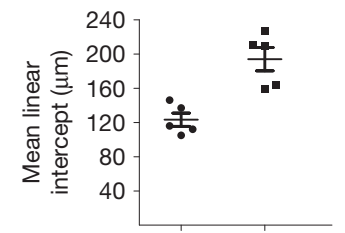

PLT infused: Sdf1+/+ Sdf1-/-

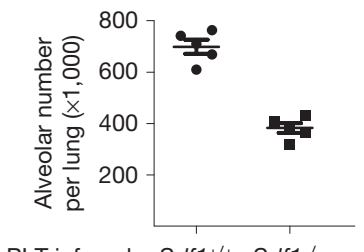

Figure 4 Intravascular transfusion of $S d f 1^{+/+}$but not $S d f 1$-deficient ( $S d f 1^{-/-}$) platelets promotes alveolar regeneration in $\mathrm{Thpo}^{-/-}$mice. (a-c) Platelets accumulate in the lungs of pneumonectomized $\mathrm{Thpo}^{-/-}$mice after intravascular infusion. Platelets were isolated from $\beta$-actin promoter-driven tdTomato (ACTB-tdTomato) mice and infused into Thpo-/- mice (a). Accumulation of tdTomato ${ }^{+} \mathrm{CD} 1^{+}$platelets in the lungs of $\mathrm{Thpo}^{-/-}$mice was determined by flow cytometry (b) and immunostaining (c); scale bar, $50 \mu \mathrm{m}$. (d-g) Strategy to examine the influence of platelet-derived SDF-1 on lung alveolar regeneration. $S d f 1^{+/+}$and $S d f 1^{-/-}$platelets were isolated from WT $\left(S d f 1^{+/+}\right)$and $S d f 1^{\Delta / \Delta}$ mice, respectively, and infused into pneumonectomized Thpo-/mice. Right lung volume (e), mass (f) and respiratory function (g) were determined in recipient mice. (e,f), $n=5$ mice in all groups; $P=0.011$ (e) and 0.026 (f) between two platelet groups. (g), $n=6$ mice $\left(S d f 1^{+/+}\right)$ and 4 mice $\left(S d f 1^{-/-}\right) . P=0.0013$ (left) and 0.0042 (right) between two groups. (h-k) Expansion of AEC2s $(\mathbf{h}, \mathbf{j})$ and PCECs $(\mathbf{i}, \mathbf{k})$ in Thpo ${ }^{-/-}$mice infused with $S d f 1^{+/+}$and $S d f 1^{-/-}$platelets. Representative immunostaining images of the two transplanted groups and flow cytometry graphs are shown; $n=5$ mice in all groups; $P=0.00011(\mathrm{j})$ and $0.00081(\mathbf{k})$ between the two platelet types. Scale bar, $50 \mu \mathrm{m}$. (I) AEC1s in $\mathrm{Thpo}^{-1-}$ mice following PNX and platelet transplantation. Scale bar, $50 \mu \mathrm{m}$. (m-0) Alveolar architecture of $\mathrm{Thpo}^{-/-}$mice receiving platelet infusion after PNX. Alveolar morphology was assessed by haematoxylin and eosin (H\&E) staining (m), and alveolar mean linear intercepts $(\mathbf{n})$ and numbers (o) were compared; $n=5$ mice in both groups; $P=0.027(\mathbf{n})$ and 0.0015 (o) between mice transplanted with the two platelet types. Scale bar, $50 \mu \mathrm{m}$. The error bar defines the s.e.m., and the line represents the mean for $\mathbf{e}-\mathbf{g}, \mathbf{j}, \mathbf{k}, \mathbf{n}$ and $\mathbf{o}$. Statistical difference between groups was assessed by an unpaired twotailed $t$-test. 


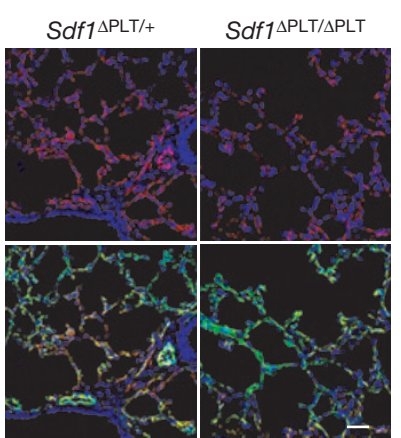

MMP14 VE-Cad Nuclei PNX day 7 b

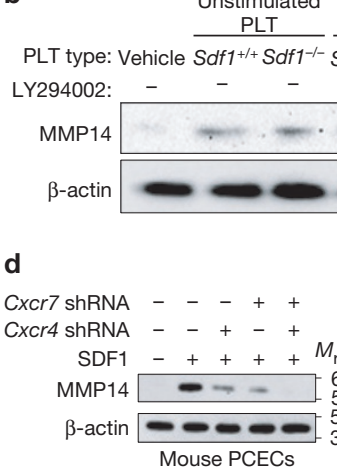

PLT type: Vehicle Sdf1 ${ }^{+/+}$Sdf1 $1^{-/} S d f 1^{+/+} S d f 1^{-/-} S d f 1^{+/+}$

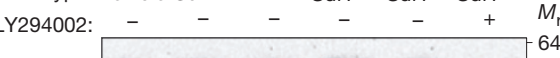

MMP14 $\beta$-actin
$M_{r}(K)$ $-64$ 51

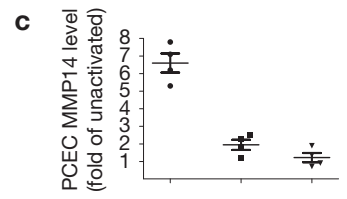

PLT type: $S d f 1^{+/+} S d f 1^{-/-} S d f 1^{+/+}$ LY294002: - $\quad$ - $\quad+$

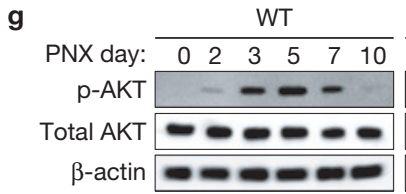

i

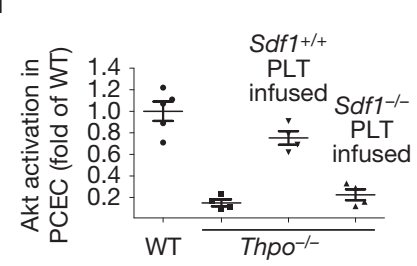

j

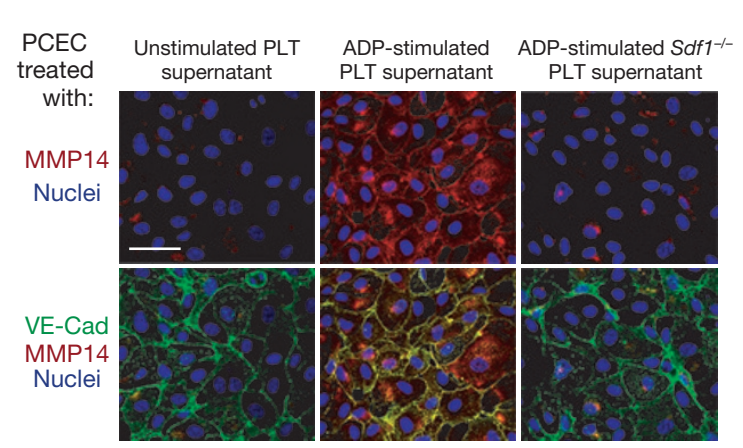

k

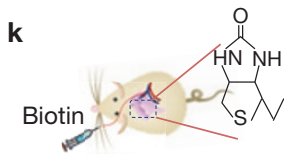

In situ biotinylation of lung endothelial cell membrane proteins Characterization of PCEC membrane
proteins during alveolar regeneration

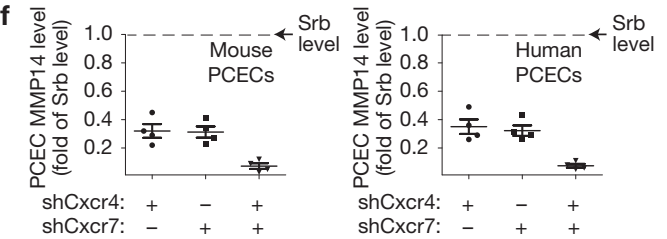

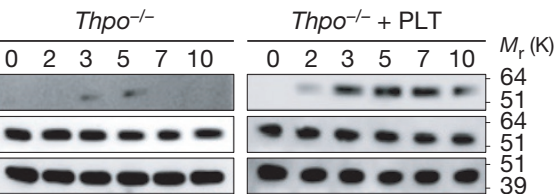

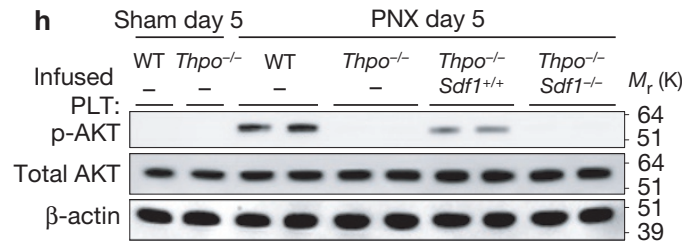

I

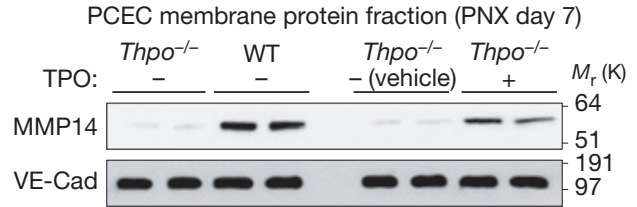

m Lung EC membrane protein fraction

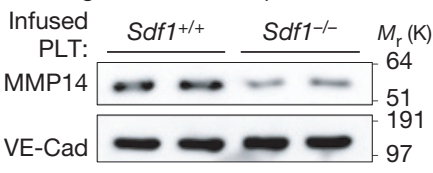

Recipient: Thpo-/-

O

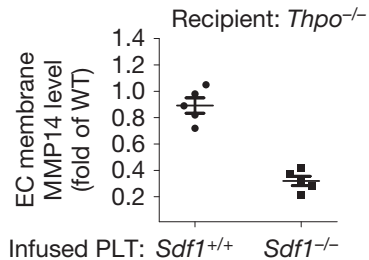

n Release of HB-EGF into alveolar space

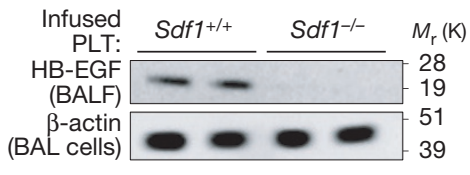

Recipient: Thpo-/-

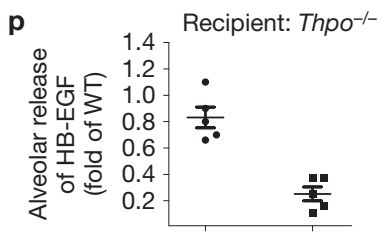

Infused PLT: Sdf1+/+ Sdf1-/-
Figure 5 Platelet-derived SDF-1 stimulates the Akt pathway to deploy membrane-type MMP14 in PCECs, leading to release of HB-EGF. (a) Expression of MMP14 in Sdf1 ${ }^{\triangle \mathrm{PLT} / \triangle \mathrm{PLT}}$ and control mice after PNX. MMP14 was co-stained with the PCEC marker VE-cadherin in lung cryosections. Scale bar, $50 \mu \mathrm{m}$. (b,c) MMP14 protein level in VE-cadherin ${ }^{+}$mouse PCECs after treatment with supernatant from indicated platelet types. The effect of PI3K inhibitor LY294002 was also tested. (c) $n=4$ cell samples in all groups. $P=0.00028$ between PCECs treated with the two platelet types. $P=0.00011$ between PCECs with or without LY294002. (d-f) The protein level of MMP14 was measured in SDF-1-stimulated PCECs. Cxcr4 and $\mathrm{Cxcr} 7$ were silenced in PCECs by short hairpin RNA (shRNA) and compared with Scrambled (Srb) transduced PCECs (dashed line). (f) $n=4$ cell samples in all groups. $P<0.01$ ( $C x c r 4$ and $C x c r 7$ shRNA groups versus Srb group). (g) Akt activation in PCECs of WT $\mathrm{Thpo}^{-1-}$ and $\mathrm{Thpo}^{-1-}$ mice receiving platelets $(+P L T)$ after PNX. The level of phosphorylated Akt (p-Akt) in isolated PCECs was detected by immunoblot. Protein levels of total Akt and $\beta$-actin served as controls for the amounts of loaded protein. (h,i) PCEC Akt activation in indicated mouse groups after infusion of platelets. (i) $n=4$ mice in all groups. $P=0.001$ between groups infused with $S d f 1^{+/+}$and $S d f 1^{-1-}$ platelets. (j) Cellular localization of MMP14 in stimulated mouse PCECs was determined by co-staining with the junction protein VE-cadherin. Scale bar, $50 \mu \mathrm{m}$. (k,I) Membrane level of MMP14 protein in PCECs of Thpo-/and WT mice after PNX. Mouse PCEC membrane proteins were biotinylated and isolated (k). The MMP14 level was measured in membrane proteins by immunoblot (I). The endothelial-specific surface marker VE-cadherin served as a protein loading control. The effect of thrombopoietin (TPO) injection on $\mathrm{Thpo}^{-/-}$mice was tested. ( $\left.\mathbf{m}-\mathbf{p}\right)$ Protein levels of PCEC membrane MMP14 $(\mathbf{m}, \mathbf{0})$ and $\mathrm{HB}-\mathrm{EGF}$ in $\operatorname{BALF}(\mathbf{n}, \mathbf{p})$ in pneumonectomized mice after platelet infusion. The $\beta$-actin protein of cells in BALF was used for normalization of HB-EGF level. $n=5$ mice per group in $\mathbf{o}$ and p. $P=0.0012(\mathbf{o})$ and 0.0014 (p) between the two platelet types. In the presented immunoblot image, each lane indicates an individual mouse or cell sample. All experiments were replicated twice with two cohorts. The error bar depicts the s.e.m. and the line represents the mean for $\mathbf{c}, \mathbf{f}, \mathbf{i}, \mathbf{o}$ and $\mathbf{p}$. The statistical difference between groups was assessed by an unpaired two-tailed $t$-test. Uncropped images of blots/gels are shown in Supplementary Fig. 9. 
a

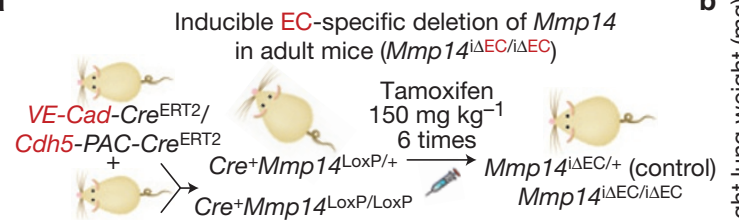

Mmp14LoxP/LoxP

d BrdU SFTPC VE-Cad DAPI

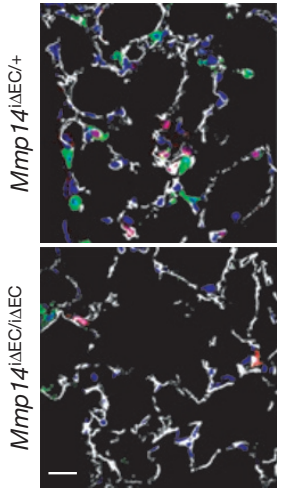

e $\quad \mathrm{PNX}$ day 7

을ㄹ 407

要促 $30-\frac{0}{\div}$

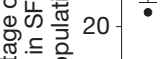

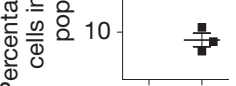

口.

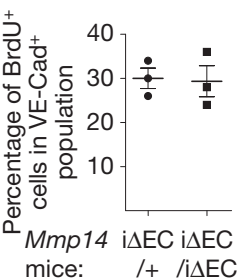

PNX day 7

j Aquaporin-5 Podoplanin VE-Cad DAPI

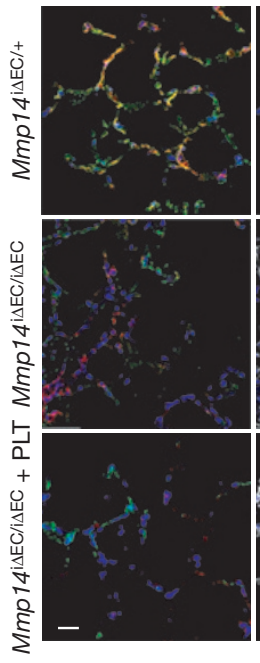

o
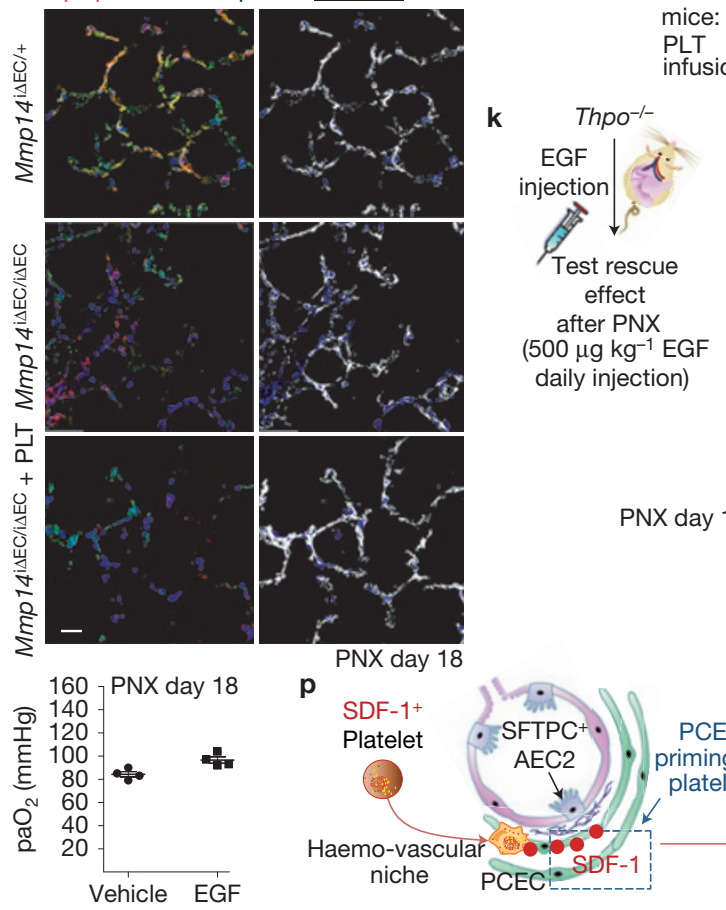

p

SDF-1+

Platelet

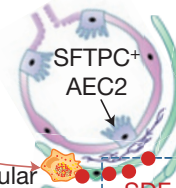

Haemo-vascular 6.00

niche

PCEC:- SDF-1

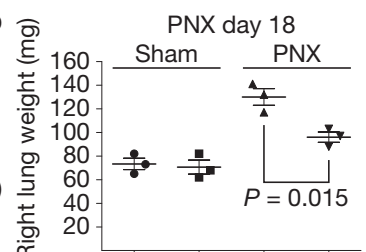

Mmp14 i $\Delta$ EC i $\Delta$ EC i $\Delta$ EC i $\Delta$ EC mice: $/+/ \mathrm{i} \Delta \mathrm{EC} /+/ \mathrm{i} \Delta \mathrm{EC}$

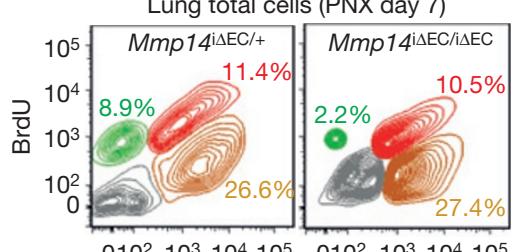

$010^{2} 10^{3} 10^{4} 10^{5} \quad 010^{2} 10^{3} 10^{4} 10^{5}$

VE-cadherin

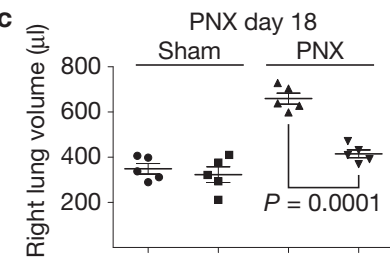

Mmp14 i $\Delta \mathrm{EC}$ i $\Delta \mathrm{EC}$ i $\Delta \mathrm{EC}$ i $\Delta \mathrm{EC}$

mice: $/+\quad / \mathrm{i} \Delta \mathrm{EC} /+\quad / \mathrm{i} \Delta \mathrm{EC}$ g

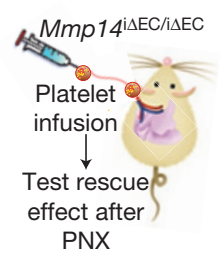

PNX

PNX day 18

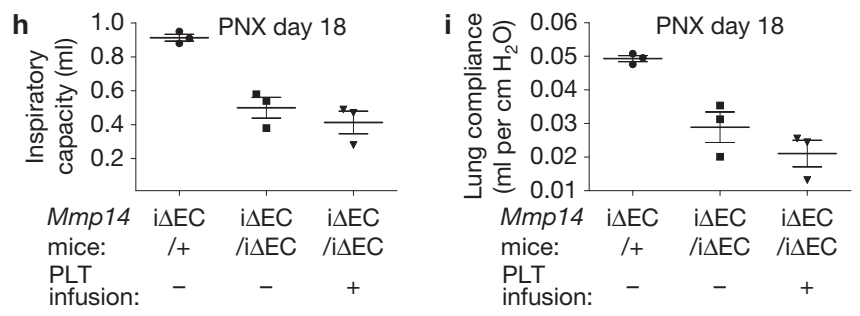

I PNX day 7

infusion:

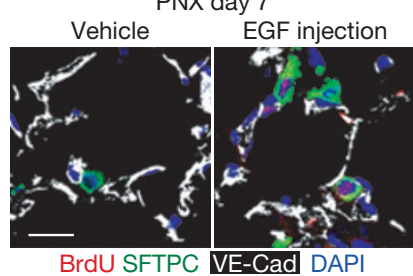

Lung total cells (PNX day 7)

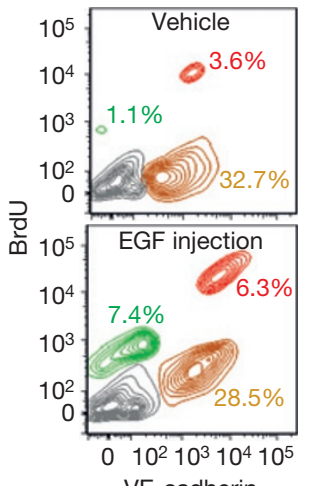

Aquaporin-5 Podoplanin DAPI

VE-cadherin

PNX day 18

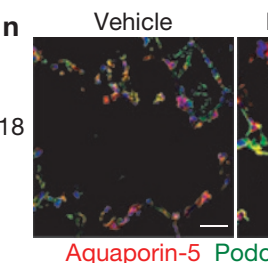

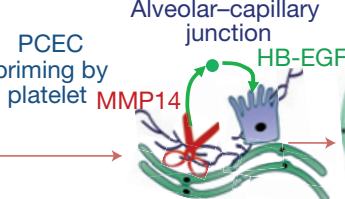

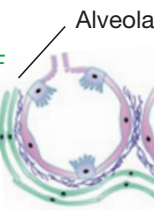

Alveolar regeneration

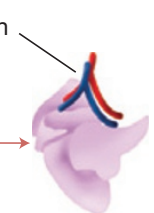

Figure 6 Expression of MMP14 in the PCEC niche is essential for evoking functional recovery of AECs after PNX. (a) EC-specific inducible deletion of Mmp14 in adult mice. Mice carrying EC-specific promoter VE-cadherindriven tamoxifen-responsive $\mathrm{Cre}\left(\mathrm{Cdh} 5-\mathrm{PAC}-\mathrm{Cr} \mathrm{e}^{\mathrm{ERT} 2}\right)$ were bred with mice harbouring LoxP site-flanked Mmp14 to generate mice with EC-specific deletion of Mmp14 (Mmp14 $\left.4^{\mathrm{\triangle EC} / \mathrm{I} \triangle \mathrm{EC}}\right)$. Mmp14 $4^{\mathrm{iEC} /+}$ mice harbouring ECspecific Mmp14 haplodeficiency were used as controls. (b,c) Lung mass (b) and volume (c) in Mmp14 $4^{\mathrm{i} E \mathrm{EC} / \mathrm{IEC}}$ and control mice after sham treatment and PNX. $n=3$ mice in all groups. (d-f) Proliferation of AEC2s (d) and PCECs (f) in Mmp14 ${ }^{\mathrm{iEC} / \mathrm{i} \triangle \mathrm{EC}}$ and control mice. (e) $n=3$ animals in all groups; $P=0.0029$ (AEC2 proliferation, top panel) and 0.88 (PCEC proliferation, bottom panel) between Mmp14 ${ }^{\mathrm{i \triangle CC} / \mathrm{i \Delta EC}}$ and control group; scale bar, $50 \mu \mathrm{m}$. (g-j) Effect of platelet infusion on the alveolarization in $\mathrm{Mmp1} 14^{\mathrm{i} \mathrm{EC} / \mathrm{SEC}}$ mice. Platelets were infused into pneumonectomized $M m p 14^{\mathrm{i} \triangle \mathrm{EC} / \mathrm{i \Delta EC}}$ mice and these were compared with control mice $(\mathbf{g})$. Recovery of pulmonary respiratory function $(\mathbf{h}, \mathbf{i})$ and AECs (j) was determined. Platelet infusion failed to rescue the defective alveolar regeneration in $M m p 14^{i \triangle E C} / \triangle \mathrm{AEC}$ mice. $n=3$ mice per group in $\mathbf{h}$ and $\mathbf{i} ; P=0.003(\mathbf{h}), 0.011$ (i) between control and $M m p 14^{\text {i } \triangle E C / I \Delta E C}$ mice; scale bar, $50 \mu \mathrm{m}$. (k-o) Influence of recombinant EGF on Thpo-/mice after PNX. EGF was injected into pneumonectomized Thpo-/- mice (k). Proliferation of AEC2s (I) and PCECs $(\mathbf{m})$, recovery of AEC1s $(\mathbf{n})$ and blood oxygenation (o) were assessed. (o) $n=4$ mice in both groups; $P=0.006$ between EGF and vehicle injected groups; scale bar, $50 \mu \mathrm{m}$. The error bar defines the s.e.m., and the line depicts the mean for $\mathbf{b}, \mathbf{c}, \mathbf{e}, \mathbf{h}, \mathbf{i}$ and $\mathbf{o}$. An unpaired two-tailed $t$-test was used to determine the statistical difference between groups. (p) Platelets activated by depositing SDF-1 activate the MMP14 pathway in PCECs, functionalizing a haemo-vascular niche that promotes lung neo-alveologenesis. After PNX, activated platelets supply SDF-1 to deploy MMP14 in the PCEC niche and the alveologenic ligand HB-EGF, eliciting propagation of AEC2s and driving alveologenesis. 


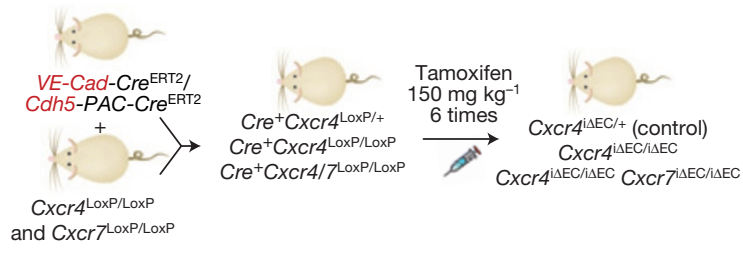

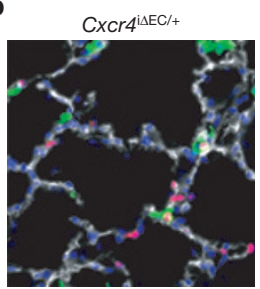

CXCr4 ${ }^{\mathrm{I} E \mathrm{EC} / \mathrm{I} E \mathrm{EC}}$
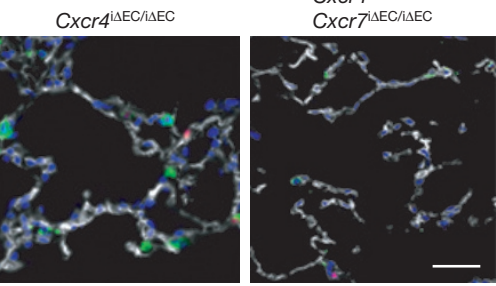

BrdU SFTPC VE-Cad DAP

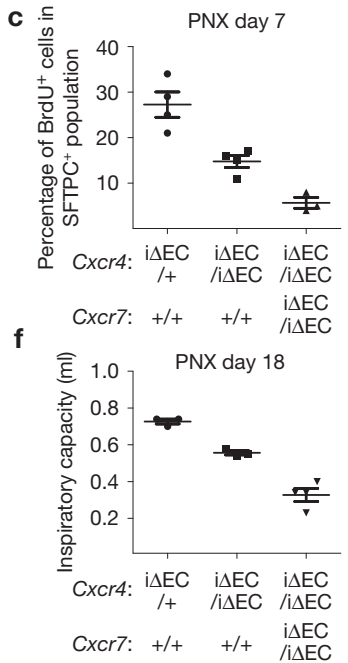

c

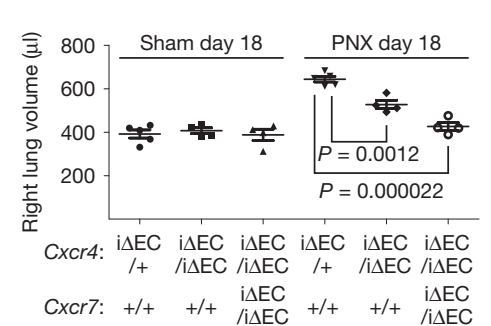

I

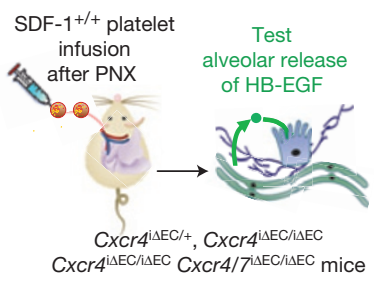

d
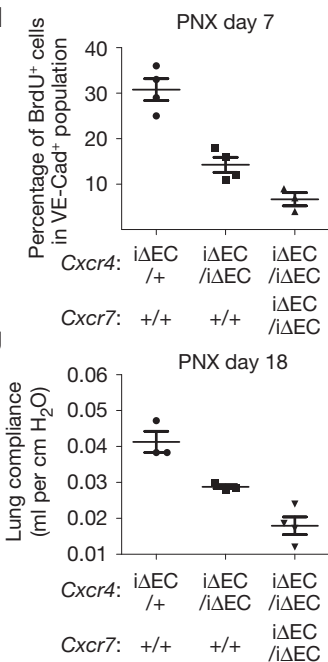

e

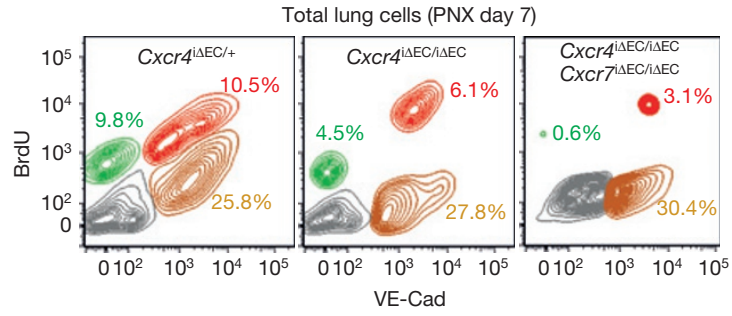

$\mathbf{h}$

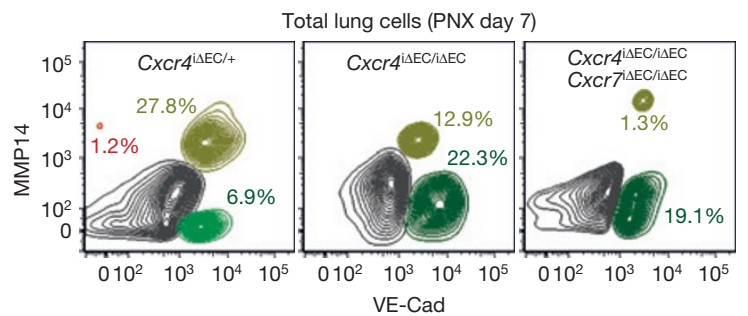

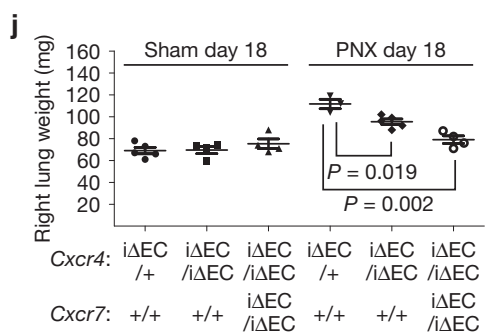

m

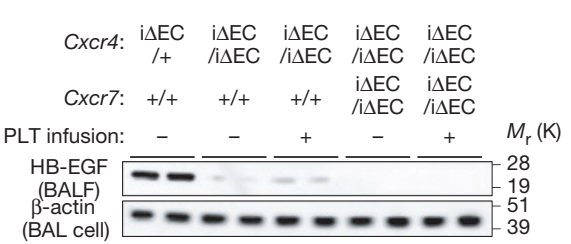

k

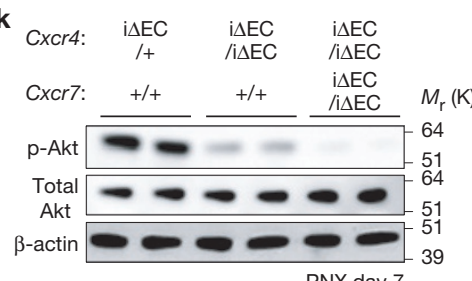

n

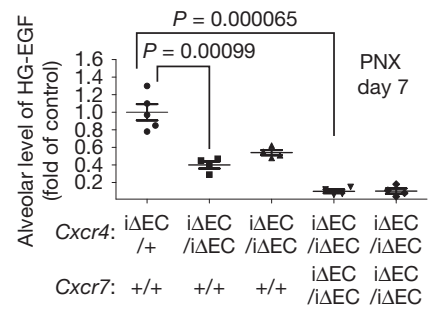

PLT infusion: -

Figure 7 Platelets activate CXCR4 and CXCR7 in the PCEC niche to drive alveolar regeneration. (a) EC-specific inducible deletion of CxCr4 and Cxcr7 in adult mice. VE-cadherin-Cre ${ }^{\mathrm{ERT} T} / \mathrm{Cdh} 5-\mathrm{PAC}-\mathrm{Cre} \mathrm{ERT}^{\mathrm{ERT}}$ mice were crossed with floxed $\mathrm{CxCr} 4$ and $\mathrm{Cxcr} 7$ mice to induce EC-

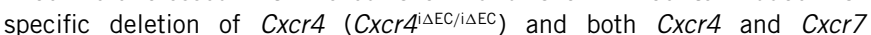

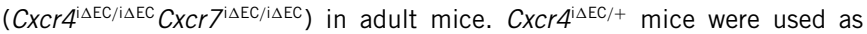
controls. (b-e) Proliferation rate of AEC2s and PCECs in control, CXCr4 $4^{\mathrm{i} E C /} / \mathrm{AEC}$ and $C X C r 4^{i \triangle E C / i \triangle E C} C X C r 7^{i \triangle E C / i \triangle E C}$ mice after PNX. $n=4$ mice in both control and $C X C r 4^{i \Delta E C / \triangle \triangle E C}$ groups, and $n=3$ mice in the $C X C r 4^{i \triangle E C / i \triangle E C} C X C r 7^{i \triangle E C / i \triangle E C}$ group; scale bar, $50 \mu \mathrm{m}$. (c) $P=0.0066$ (control versus $C x C r 4^{\mathrm{i} \triangle \mathrm{EC} / \mathrm{i} \triangle \mathrm{EC}}$ ) and 0.0015 (control versus $C X C r 4^{\mathrm{i} E \mathrm{EC} /} / \mathrm{\triangle EEC} C X C r 7^{\mathrm{I} \triangle \mathrm{EC} / \mathrm{i \triangle EC}}$ ). (d) $P=0.0013$ (control versus

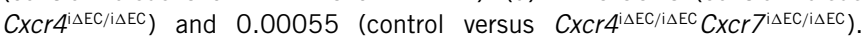
$(\mathbf{f}, \mathbf{g})$ Functional alveolar regrowth in indicated mouse groups following PNX. $(\mathbf{f}, \mathbf{g}) n=3$ mice in both control and CXCr4 ${ }^{\mathrm{iLE} / \mathrm{ALEC}}$ groups; $n=4$ mice in $C X C r 4^{\mathrm{i} E \mathrm{EC} / \mathrm{i \triangle EC}} \mathrm{CXCr} 7^{\mathrm{i} \triangle \mathrm{EC} / \mathrm{i \triangle EC}}$ group. $P=0.00069$ (f) and 0.014 (g) between control and $C X C r 4^{\mathrm{I} E \mathrm{EC} / \mathrm{IAEC}}$ mice. $P=0.00026(\mathbf{f})$ and $0.0017(\mathbf{g})$ between control and $C X C r 4^{\mathrm{i} \triangle \mathrm{EC} / \mathrm{i \triangle EC} C X C r} 7^{\mathrm{i} \triangle \mathrm{EC} / \mathrm{PEEC}}$ groups. (h) The PCEC MMP14 protein level in control, $C X C r 4^{\mathrm{i} E \mathrm{EC} / \mathrm{AEC}}$ and $C X C r 4^{\mathrm{i} \triangle \mathrm{EC} / \mathrm{i \triangle EC} C X C r} 7^{\mathrm{i} \triangle \mathrm{EC} / \mathrm{i \triangle EC}}$ mice was tested by flow cytometry. (i-k) Weight (i), volume (j) and PCEC Akt activation (k) in right lungs after PNX. Immunoblotting was used to test the phosphorylated Akt (p-Akt) level in PCECs. $n=5,4,4,5,4,4$ mice (i) and $5,4,4,3,5,4$ animals $(j)$ in control $C X C r 4^{i \triangle E C /+}, C X C r 4^{i \triangle E C / i \triangle E C}$ and

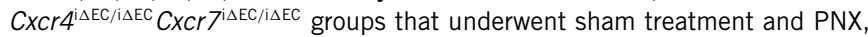
respectively. (I-n) BALF level of HB-EGF in pneumonectomized $C X C r 4^{i \Delta E C / i \Delta E C}$ and $C x C r 4^{i \Delta E C / i \Delta E C} C x C r 7^{i \triangle E C / i \Delta E C}$ mice after infusion of $S d f 1^{+/+}$platelets. The influence of platelets on alveolar regeneration of the indicated mouse groups was investigated as described in I; HB-EGF in BALF was tested by immunoblot (m) and quantified (n). (n) $n=5$ mice in control $C x C r 4^{i \triangle E C /+}$ group, $n=4$ mice in the other shown groups. In the shown immunoblot image, each lane represents a sample from an individual mouse. Experiments were carried out with mice from two cohorts. The error bar denotes the s.e.m., and the line defines the mean for $\mathbf{c}, \mathbf{d}, \mathbf{f}, \mathbf{g}, \mathbf{i}, \mathbf{j}$ and $\mathbf{n}$. Statistical differences between groups were analysed by unpaired two-tailed $t$-tests. Uncropped images of blots/gels are shown in Supplementary Fig. 9. 

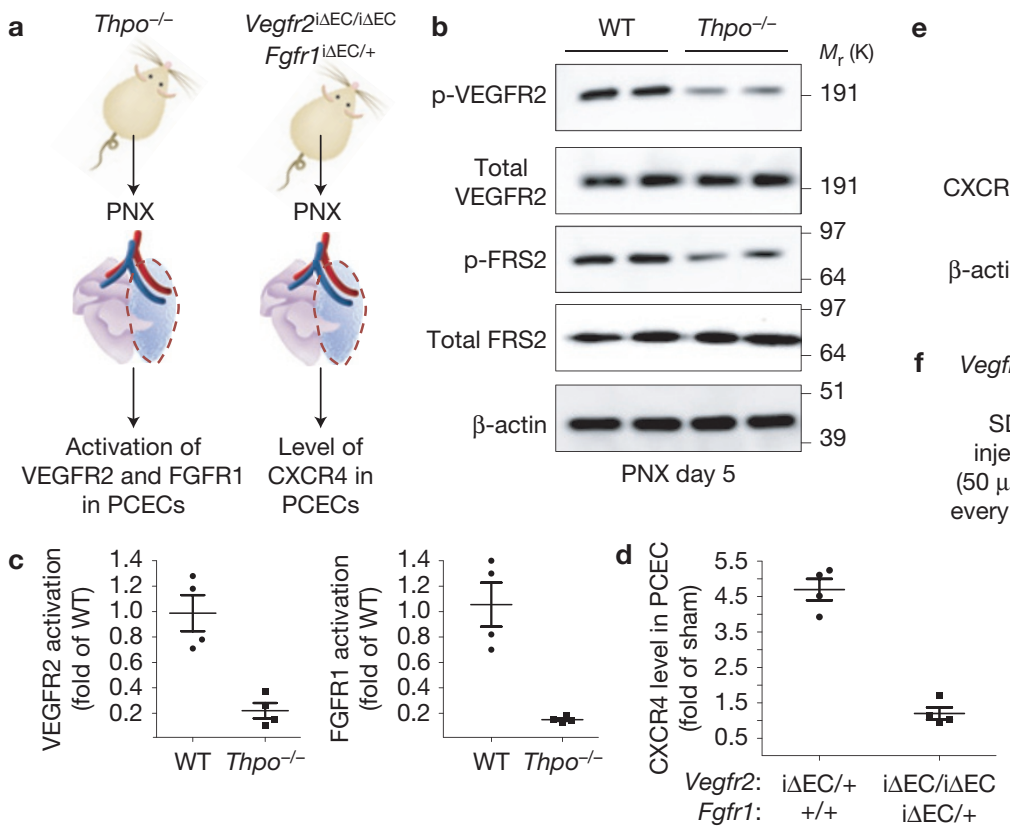

h

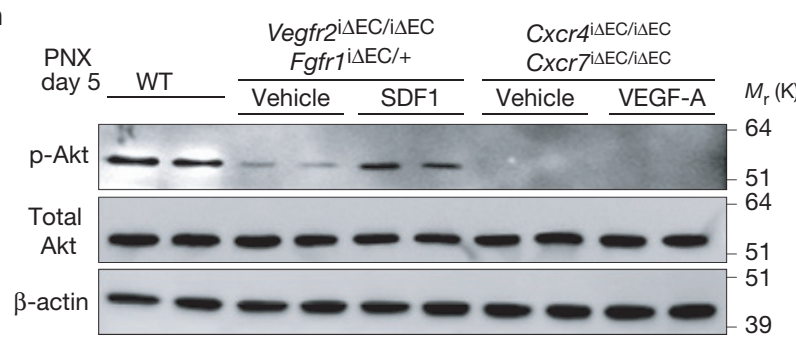

j

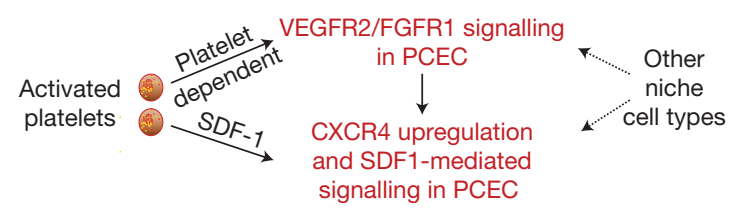

e

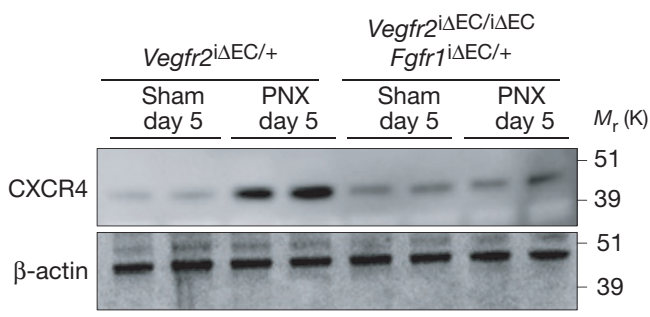

f Vegfr $2^{\mathrm{i} \Delta \mathrm{EC} / \mathrm{i} \Delta \mathrm{EC}} F_{g f r} 1^{\mathrm{i} \Delta \mathrm{EC} /+} \quad C x c r 4^{\mathrm{i} \Delta \mathrm{EC} / \mathrm{i} \Delta \mathrm{EC}} \mathrm{Cxcr} 7^{\mathrm{i} \Delta \mathrm{EC} / \mathrm{i} \Delta \mathrm{EC}}$

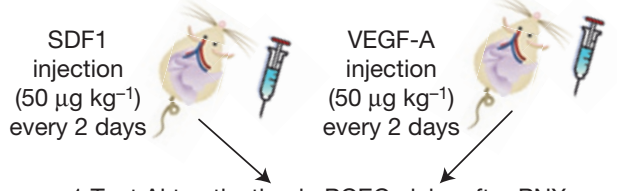

1. Test Akt activation in PCEC niche after PNX 2.Test lung function restoration after PNX

g
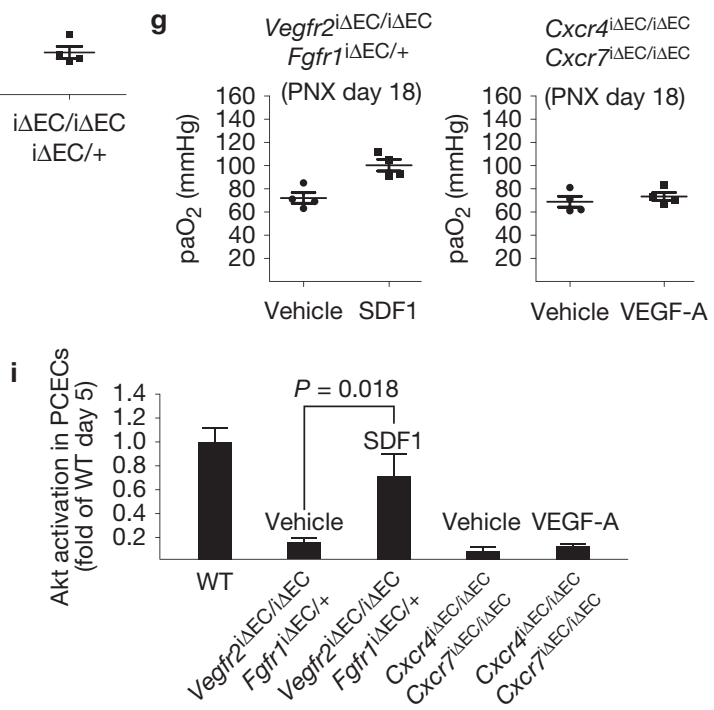

$\mathbf{k}$

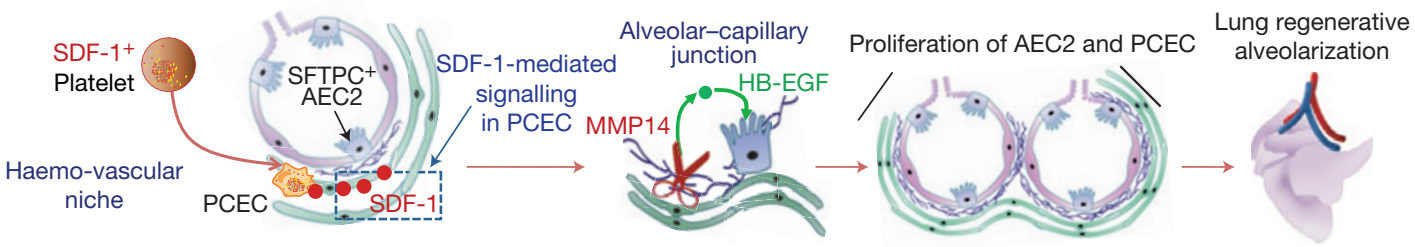

Figure 8 Regulation of SDF-1 signalling in PCEC niche by VEGFR2/FGFR1 pathways. (a) Strategy to test the activation of VEGFR2 and FGFR1 and expression of SDF-1 receptor CXCR4 in PCECs of the indicated mouse groups. (b,c) Activation of VEGFR2 and FGFR1 in PCECs of Thpo-/- mice after PNX. Phosphorylation of VEGFR2 ( $\mathrm{p}-\mathrm{VEGFR2)}$ was tested by immunoblotting. To test FGFR1 activation, phosphorylation of the downstream effector FGF receptor substrate 2 ( $p$-FRS2) was similarly assessed. Protein levels of VEGFR2 and FRS2 and $\beta$-actin were also measured as controls. $n=4$ mice in all tested groups; $P=0.0172$ (VEGFR2 activation) and 0.0153 (FGFR1 activation) between WT and Thpo $^{-1-}$ mice. (d,e) Expression of CXCR4 protein in mice that are deficient in

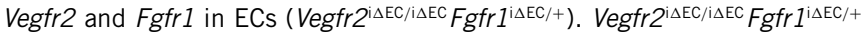
mice were generated as previously described ${ }^{1} . n=4$ animals in both groups, and $P=0.0005$ between the two genotypes. $(\mathbf{f}, \mathbf{g})$ Rescue effect of VEGF $_{164}$ and SDF-1 in pneumonectomized $C x C r 4^{\mathrm{i} \triangle \mathrm{EC} / \mathrm{i} \Delta \mathrm{EC}} \mathrm{CxCr} 7^{\mathrm{i} \Delta \mathrm{EC} / \mathrm{i \Delta EC}}$ and Vegfr2 ${ }^{\mathrm{i} \triangle \mathrm{EC} / \mathrm{i} \triangle \mathrm{EC}} \mathrm{Fgfr}^{\mathrm{i} \triangle \mathrm{EC} /+}$ mice, respectively. Restoration of gas exchange function (f) and PCEC Akt activation (g) were tested. $P=0.0319$ between

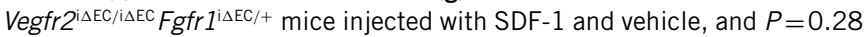

between $C X C r 4^{\mathrm{i} \triangle \mathrm{EC} / \mathrm{i} \mathrm{ECC}} C X C r 7^{\mathrm{i} \Delta \mathrm{EC} / \mathrm{i} \Delta \mathrm{EC}}$ mice injected with $\mathrm{VEGF}_{164}$ and vehicle; $n=4$ mice per group. (h,i) PCEC Akt activation in the indicated mice was determined. $n=6$ mice in all tested groups. In the presented immunoblot image, each lane indicates an individual mouse sample. Mice from two different cohorts were tested. The error bar stands for the s.e.m., and the line represents the mean for $\mathbf{c}, \mathbf{d}, \mathbf{g}$ and $\mathbf{i}$. The statistical difference between groups was determined by an unpaired two-tailed $t$-test. (j) Regulation of SDF-1 signalling in the PCEC niche by the VEGFR2/FGFR1 pathways after PNX. Activation of VEGFR2 and FGFR1 is mediated by a platelet-dependent mechanism. Activation of VEGFR2/FGFR1 synergistically acts with SDF-1 receptors (CXCR4/CXCR7) on PCECs to trigger vascular niche-mediated neo-alveologenesis. (k) Platelets and PCECs functionalize a haemo-vascular niche to drive lung alveolar regeneration/regrowth. On PNX, activated platelets supply SDF-1 to activate CXCR4 and CXCR7 on PCECs. Platelet-mediated CXCR4/7-Akt activation deploys angiocrine MMP14 in PCECs and causes release of the alveologenic ligand HB-EGF, eliciting propagation of AEC2s and driving neo-alveologenesis. Uncropped images of blots/gels are shown in Supplementary Fig. 9. 
littermates. In WT mice, PNX enhanced the mass, volume and respiratory function of the residual right lungs to a level comparable to those of the unresected sham lungs after 18 days (Fig. 1c-e). Notably, there was minor expansion of activated lung fibroblast cells in the residual right lungs after PNX, implicating the absence of fibrosis (Supplementary Fig. 1). By contrast, this functional regrowth of the lungs was markedly reduced in Thpo-l- mice (Fig. 1c-e). PNX-induced alveolarization is driven by the propagation of pro-surfactant protein $\mathrm{C}\left(\mathrm{SFTPC}^{+}\right)$AEC2s (refs 9,10) and VE-cadherin $^{+}$PCECs, which peaks at day 7 after PNX (ref. 1). Using bromodeoxyuridine (BrdU) incorporation, we found that the proliferation of AEC2s and PCECs induced by PNX was significantly lower in Thpo-/- mice than WT mice (Fig. 1f-i, Supplementary Figs 1 and 2a). Reduced AEC2 proliferation in Thpo-/- mice was associated with lower restoration of aquaporin $5^{+}$podoplanin ${ }^{+}$type I alveolar epithelial cells (AEC1s) in the pneumonectomized lungs (Fig. 1j), underscoring the potential of platelets to promote lung alveolar regeneration.

We then injected anti-CD41 monoclonal antibody (mAb) into WT mice to deplete platelets and subjected these thromobocytopenic mice to PNX (Fig. 1k and Supplementary Fig. 2). Rat IgG-treated mice were used as control. After PNX, recovery of volume, expansion of AECs and PCECs, and blood oxygenation were all inhibited in the remaining lungs of CD41 mAb-treated mice after PNX (Fig. 1l-q). Therefore, circulating platelets are essential for restoring the cellular components and respiratory function in the lungs after PNX.

\section{Platelets deposit SDF-1 (CXCL12) to mediate PNX-induced neo-alveolarization}

Activated platelet cells deploy chemokines such as stromal cellderived factor-1 (SDF-1, also known as CXCL12) to promote organ repair $^{24,34,40}$. Indeed, we found that the majority of $\mathrm{CD} 41^{+}$platelets associated with PCECs were stained as SDF- $1^{+}$in the lung cryosection after PNX but not sham operation (Fig. 2a and Supplementary Fig. 2e). This unexpected deposition of SDF- $1^{+} \mathrm{CD} 41^{+}$platelets on VE-cadherin ${ }^{+}$PCECs reached the highest level at day 3 and was sustained for at least 9 days after PNX (Fig. 2b,c). SDF-1 belongs to the CXC chemokine family ${ }^{4-49}$ and plays a key role in modulating organogenesis $^{50-53}$. Indeed, SDF-1 injection significantly enhanced the recovery of AECs and restoration of gas exchange function in pneumonectomized Thpo ${ }^{-/-}$mice (Fig. 2d-f). To examine the role of SDF-1 in PNX-induced lung regrowth, we conditionally deleted the $S d f 1$ gene by crossing $S d f 1^{\text {LoxP/LoxP }}$ mice ${ }^{53}$ with tamoxifen-responsive ROSA-Cre $e^{\text {ERT2 }}$ mice. Treatment of the offspring with tamoxifen led to inducible deletion of $S d f 1$ in adult mice ( $S d f 1^{\Delta / \Delta}$; Fig. $2 \mathrm{~g}-\mathrm{i}$ ). Compared with control mice, $S d f 1^{\Delta / \Delta}$ mice exhibited impaired AEC2 propagation and mitigated restoration of lung weight and volume after PNX (Fig. 2j,k). Thus, SDF-1 signalling is critical for lung alveolar regeneration induced by $\mathrm{PNX}$.

We then formally deciphered the functional contribution of SDF-1 from various niche cell components to lung regeneration (Supplementary Fig. 3). The in vivo role of platelet-derived SDF1 was examined by breeding a Platelet factor 4-Cre mouse line with floxed $S d f 1$ mice (Fig. 3a). This strategy specifically deletes $S d f 1$ from mouse platelets and platelet progenitors $\left(S d f 1^{\Delta \mathrm{PLT} / \Delta \mathrm{PLT}}\right)$.
Compared with control $S d f 1^{\Delta \mathrm{PLT} /+}$ mice, the restoration of lung mass and volume and regeneration of AECs and PCECs were significantly reduced in $S d f 1^{\triangle \mathrm{PLT} / \Delta \mathrm{PLT}}$ mice (Fig. $3 \mathrm{~b}-\mathrm{j}$ ). By contrast, deletion of $S d f 1$ from both myeloid and endothelial cells caused an insignificant reduction in tested lung regenerative responses (Supplementary Fig. 3). Notably, $S d f 1^{\triangle \mathrm{PLT} / \Delta \mathrm{PLT}}$ mice did not exhibit altered production of growth factors from platelets, recruitment of haematopoietic cells or pulmonary fibrin deposition after lung injury (Supplementary Fig. 4). Hence, deployment of SDF-1 from activated platelets is indispensable to elicit regenerative alveolarization after PNX (Fig. 3k).

\section{Intravascular infusion of SDF-1 ${ }^{+}$platelets rescues impaired alveolar regeneration in $\mathrm{Thpo}^{-/-}$mice}

The importance of platelets in evoking lung regeneration led us to postulate that infusion of platelets into $\mathrm{Thpo}^{-/-}$mice would rescue the defective regeneration. To this end, we adopted a platelet-infusion model $^{27}$ to intravenously transfuse platelets into mice (Fig. 4a). After jugular vein infusion, platelets preferentially accumulated in the lungs of Thpo-/- mice after PNX (Fig. 4b,c). As such, this platelet-infusion strategy allows for a 'gain of function' study to interrogate the influence of platelets on lung alveolar regeneration.

We then compared the effects of $S d f 1^{+/+}$and $S d f 1$-deficient $\left(S d f 1^{-/-}\right)$platelets on the impaired lung regeneration in $T h p o^{-/-}$mice (Fig. 4d). $S d f 1^{-/-}$and $S d f 1^{+/+}$platelets were isolated from $S d f 1^{\Delta / \Delta}$ and WT littermates and infused into pneumonectomized Thpo-/mice (Supplementary Fig. 5). Whereas after PNX similar numbers of infused platelets adhered to PCECs, infusion of $S d f 1^{+/+}$but not $S d f 1^{-/-}$platelets into $T h p o^{-/-}$mice rescued the restoration of lung mass and volume (Fig. 4e,f) and pulmonary function (Fig. 4g), as well as expansion of AECs and PCECs (Fig. 4h-1). Consequently, $S d f 1^{+/+}$ platelet infusion to Thpo-/- mice induced significantly elevated neoalveologenesis when compared with $S d f 1^{-/-}$platelet transplantation, as shown by higher alveolar number and lower mean linear intercept (Fig. $4 \mathrm{~m}, \mathrm{n}, \mathrm{o}$ ). Thus, increased bioavailability of SDF-1 from the infused platelets rescued the defective neo-alveolarization in pneumonectomized $\mathrm{Thpo}^{-/-}$mice.

\section{Platelet-derived SDF-1 induces membrane MMP14 in PCECs to release EGF-like ligand, stimulating neo-alveologenesis}

Induction of membrane MMP14 in PCECs by PNX causes release of EGF-like ligands such as heparin-binding EGF-like growth factor (HB-EGF; ref. 22) to stimulate the propagation of adjacent AEC2s. Close association between PCECs and recruited platelets after PNX suggests that platelets stimulate alveolar regeneration by priming the pro-regenerative function of the PCEC niche $e^{1,15,16,25,54}$. Indeed, PCEC expression of MMP14 in $S d f 1^{\triangle \mathrm{PLT} / \triangle \mathrm{PLT}}$ mice was significantly lower than that in control mice after PNX (Fig. 5a). Thus, we analysed how platelet SDF-1 regulates PCEC deployment of MMP14. The supernatant of ADP-stimulated $S d f 1^{+/+}$but not $S d f 1^{-/-}$platelets caused membrane MMP14 upregulation in PCECs, which was blunted by the phosphatidylinositol 3-kinase (PI3K) inhibitor LY294002 (Fig. 5b,c). MMP14 was similarly upregulated in both mouse and human PCECs after stimulation of recombinant SDF-1, and this upregulation was abrogated by genetic silencing of SDF-1 receptors CXcr4 and CXcr7 (also known as Ackr3) in PCECs (Fig. 5d-f). These 
data imply that platelets release SDF-1 to induce pro-regenerative MMP14 in the PCEC niche, igniting regeneration without causing fibrosis (Supplementary Fig. 5a).

Blocking induction of endothelial MMP14 with PI3K inhibitor raised the hypothesis that, after PNX, platelets deploy SDF-1 to stimulate Akt-dependent MMP14 upregulation. To test this notion, we measured the Akt activation/phosphorylation in PCECs of pneumonectomized WT and Thpo-/- mice (Fig. 5g). PNX caused a time-dependent Akt phosphorylation in the PCECs of WT mice. By contrast, this PCEC Akt activation by PNX was significantly lower in $\mathrm{Thpo}^{-/-}$mice, which was enhanced by infusion of $S d f 1^{+/+}$rather than $S d f 1^{-/-}$platelets (Fig. 5h,i). These findings suggest that deposition of SDF-1 by platelets stimulates Akt-dependent upregulation of MMP14 in PCECs after PNX.

Cellular surface localization of MMP14 is critical for the protease activity of membrane-type MMP14 (refs 20,22,23). Treatment of PCECs with supernatant from activated platelets stimulated SDF-1dependent membrane localization of MMP14 protein (Fig. 5j). To determine the distribution of MMP14 in regenerating PCECs, we used an in situ pulmonary vascular perfusion system to selectively biotinylate and fractionate PCEC membrane proteins (Fig. 5k and Supplementary Fig. 6a) ${ }^{55}$. At day 7 after PNX, the MMP14 level in the isolated PCEC membrane proteins was significantly higher in the pneumonectomized lungs of WT but not Thpo-l- mice (Fig. 5l). Both thrombopoietin (TPO) injection and transplantation of WT platelets to Thpo-/- mice augmented membrane enrichment of MMP14 in PCECs (Fig. 5m). Subsequently, release of an epithelially active substrate of MMP14, HB-EGF, in the alveolar space was augmented in $T h \mathrm{po}^{-/-}$mice transplanted with $S d f 1^{+/+}$ but not $S d f 1^{-/-}$platelets, as shown by an elevated HB-EGF protein level in bronchioalveolar lavage fluid (BALF; Fig. 5n-p). These findings indicate that SDF-1 from activated platelets primes the PCEC niche to deploy membrane MMP14, initiating propagation of adjacent AECs.

\section{Inducible endothelial-specific deletion of Mmp14 in mice blocked neo-alveologenesis after PNX}

We then used an EC-specific genetic deletion strategy to assess the contribution of endothelial-derived MMP14 in stimulating neo-alveologenesis. Tamoxifen-inducible EC-specific mouse deleter

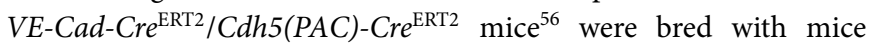
harbouring LoxP-site-flanked Mmp14 (ref. 21). Intraperitoneal injection of tamoxifen into resultant offspring induced EC-specific Mmp14 ablation $\left(M m p 14^{\mathrm{i} \Delta \mathrm{EC} / \mathrm{i} \Delta \mathrm{EC}}\right.$ ) in adult mice (Fig. 6a and Supplementary Fig. 6c,d). After PNX, restoration of lung mass and volume and cellular propagation were markedly reduced

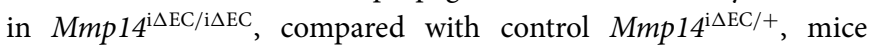
(Fig. 6b-f). Notably, infusion of platelets failed to rescue the impaired functional alveolar regrowth in $M m p 14^{\mathrm{i} \triangle \mathrm{EC} / \mathrm{i} \triangle \mathrm{EC}}$ mice after PNX (Fig. 6g-j). By contrast, injection of MMP14 downstream effector recombinant EGF into $T h p o^{-1-}$ mice rescued the defective lung regeneration after PNX (Fig. 6k-o and Supplementary Fig. 6e,f). These data suggest that recruited platelets after PNX deploy MMP14 in the PCEC niche to stimulate EGF receptor (EGFR) signalling in proximal AEC2s and instigate their expansion, driving neo-alveologenesis and restoration of pulmonary function (Fig. 6p).

\section{SDF-1 receptors CXCR4 and CXCR7 are required for induction of alveologenic MMP14 in PCECs}

To examine the molecular mechanism whereby platelet SDF-1 activates the PCEC niche, we tested the contribution of SDF-1 receptors CXCR4 and CXCR7 on PCECs after PNX. Injection of CXCR4 antagonist AMD3100 blocked cell proliferation and lung function recovery following PNX (Supplementary Fig. 7), suggesting the importance of CXCR4 in promoting PNX-induced alveolar regrowth. To circumvent the vascular defect and embryonic lethality caused by $C x c r 4$ genetic deletion ${ }^{48}$, we inducibly deleted Cxcr4 and Cxcr7 from the ECs of adult mice $\left(C x c r 4^{\mathrm{i} \triangle \mathrm{EC} / \mathrm{i} \Delta \mathrm{EC}}\right.$ and $C x c r 4^{\mathrm{i} \triangle \mathrm{EC} / \mathrm{i} \Delta \mathrm{EC}} C x c r 7^{\mathrm{i} \triangle \mathrm{EC} / \mathrm{i} \Delta \mathrm{EC}} ;$ Fig. $7 \mathrm{a}$ and Supplementary Fig. 8a,b). Alveolar regeneration in $C x c r 4^{\mathrm{i} \Delta \mathrm{EC} / \mathrm{i} \Delta \mathrm{EC}}$ mice was significantly impaired when compared with control $C x c r 4^{i \Delta \mathrm{EC} /+}$ mice, including expansion of AECs and PCECs (Fig. 7b-e), recovery of lung function (Fig. 7f,g), MMP14 induction in PCECs (Fig. 7h) and restoration of mass and volume (Fig. 7i,j). Of note, this defect in functional alveolar regrowth was further exacerbated in $C x c r 4^{\mathrm{i} \triangle \mathrm{EC} / \mathrm{i} \Delta \mathrm{EC}} C x c r 7^{\mathrm{i} \Delta \mathrm{EC} / \mathrm{i} \Delta \mathrm{EC}}$ mice. Impaired lung regeneration in $C x c r 4^{\mathrm{i} \Delta \mathrm{EC} / \mathrm{i} \Delta \mathrm{EC}}$ and $C x c r 4^{\mathrm{i} \Delta \mathrm{EC} / \mathrm{i} \Delta \mathrm{EC}} C x c r 7^{\mathrm{i} \Delta \mathrm{EC} / \mathrm{i} \Delta \mathrm{EC}}$ mice was associated with attenuation of Akt activation in PCECs (Fig. 7k and Supplementary Fig. 8c). To illustrate the influence of platelets on SDF-1-mediated signalling in the PCEC niche, we infused platelets into $C x c r 4^{\mathrm{i} \triangle \mathrm{EC} / \mathrm{i} \Delta \mathrm{EC}}$ and $C x c r 4^{\mathrm{i} \Delta \mathrm{EC} / \mathrm{i} \Delta \mathrm{EC}} C x c r 7^{\mathrm{i} \Delta \mathrm{EC} / \mathrm{i} \Delta \mathrm{EC}}$ mice and tested the regenerative response (Fig. 7l). Infusion of SDF- $1^{+}$platelets into mice did not restore the alveolar release of HB-EGF in either $C x c r 4^{\mathrm{i} \Delta \mathrm{EC} / \mathrm{i} \Delta \mathrm{EC}}$ or $C x c r 4^{\mathrm{i} \Delta \mathrm{EC} / \mathrm{i} \Delta \mathrm{EC}} C x c r 7^{\mathrm{i} \Delta \mathrm{EC} / \mathrm{i} \Delta \mathrm{EC}}$ mice (Fig. $7 \mathrm{~m}, \mathrm{n}$ ). Taken together, these findings suggest that platelets deploy SDF-1 to trigger CXCR4 and CXCR7 signalling in PCECs, enabling a pro-regenerative niche that drives regenerative alveolarization after PNX.

\section{Modulation of SDF-1 signalling in the pulmonary vascular niche by the VEGFR2/FGFR1 pathways}

Activation of PCECs during alveolarization is a coordinated process that requires synergistic effects of various signalling molecules, including the angiogenic factors vascular endothelial growth factor-A (VEGF-A) and fibroblast growth factors (FGFs; ref. 1). Indeed, activation of both VEGF receptor 2 (VEGFR2) and FGF receptor 1 (FGFR1) pathways was attenuated in PCECs of Thpo-/- mice after PNX (Fig. 8a-c). This finding implies that activation of VEGFR2 and FGFR1 in PCECs after PNX is at least partially dependent on platelets. To test the correlation of SDF-1 signalling with the VEGFR2/FGFR1 pathways, we tested the expression of CXCR4 in mice with EC-specific deletion of VEGFR2 and FGFR1 (ref. 1; Vegfr2 ${ }^{\mathrm{i} \Delta \mathrm{EC} / \mathrm{i} \Delta \mathrm{EC}} \mathrm{Fgfr} 1^{\mathrm{i} \Delta \mathrm{EC} /+}$ ) after PNX (Fig. 8d,e). Notably, the protein level of CXCR4 in PCECs was significantly upregulated by PNX in control but not Vegfr $2^{\mathrm{i} \triangle \mathrm{EC} / \mathrm{i} \Delta \mathrm{EC}} \mathrm{Fgfr} 1^{\mathrm{i} \triangle \mathrm{EC} /+}$ mice. These data suggest that SDF-1 signalling (receptor CXCR4) in PCECs is regulated by the previously shown pro-regenerative VEGFR2 and FGFR1 pathways after PNX.

We then used $V e g f r 2^{\mathrm{i} \Delta \mathrm{EC} / \mathrm{i} \Delta \mathrm{EC}} \mathrm{Fgfr} 1^{\mathrm{i} \Delta \mathrm{EC} /+}$ mice and $C x c r 4^{\mathrm{i} \Delta \mathrm{EC} / \mathrm{i} \Delta \mathrm{EC}} C x c r 7^{\mathrm{i} \Delta \mathrm{EC} / \mathrm{i} \Delta \mathrm{EC}}$ mice to reveal the functional regulation of SDF-1 signalling by VEGFR2/FGFR1 in the PCEC niche after PNX (Fig. 8f). First, restoration of lung function (blood oxygenation) was significantly improved in $\operatorname{Vegfr} 2^{\mathrm{i} \Delta \mathrm{EC} / \mathrm{i} \Delta \mathrm{EC}} \mathrm{Fg} f r 1^{\mathrm{i} \Delta \mathrm{EC} /+}$ mice by SDF-1 injection after PNX. By contrast, VEGF injection into 
$C x c r 4^{\mathrm{i} \Delta \mathrm{EC} / \mathrm{i} \Delta \mathrm{EC}} C x c r 7^{\mathrm{i} \Delta \mathrm{EC} / \mathrm{i} \Delta \mathrm{EC}}$ mice failed to enhance the recovery of lung function in mice (Fig. 8g). The different effects caused by VEGF-A and SDF-1 injection implicate that CXCR4/7-mediated SDF-1 signalling in PCECs is downstream of the VEGFR2 pathway.

This potentiation of SDF-1 signalling by the VEGFR2/FGFR1 pathways was further demonstrated by the analysis of Akt activation in PCECs (Fig. 8h,i). Compared with WT mice, Akt activation in PCECs was blocked in both $\operatorname{Vegfr} 2^{\mathrm{i} \Delta \mathrm{EC} / \mathrm{i} \Delta \mathrm{EC}} \mathrm{Fg} f r 1^{\mathrm{i} \Delta \mathrm{EC} /+}$ and $C x c r 4^{\mathrm{i} \Delta \mathrm{EC} / \mathrm{i} \Delta \mathrm{EC}} C x c r 7^{\mathrm{i} \Delta \mathrm{EC} / \mathrm{i} \Delta \mathrm{EC}}$ mice. Of note, whereas SDF-1 injection into Vegfr $2^{\mathrm{i} \Delta \mathrm{EC} / \mathrm{i} \Delta \mathrm{EC}} \mathrm{Fgfr} 1^{\mathrm{i} \Delta \mathrm{EC} /+}$ mice restored PCEC Akt activation, Akt activation in PCECs of $C x c r 4^{\mathrm{i} \Delta \mathrm{EC} / \mathrm{i} \Delta \mathrm{EC}} C x c r 7^{\mathrm{i} \Delta \mathrm{EC} / \mathrm{i} \Delta \mathrm{EC}}$ mice was not enhanced by VEGF-A injection. Thus, activation of VEGFR2 and FGFR1 promotes SDF-1-CXCR4/7 signalling to stimulate activation of the PCEC niche, driving the regenerative alveolarization process (Fig. 8j), and it is plausible that, after PNX, platelet-derived SDF-1 cooperates with other pathways to synergistically prime pro-regenerative PCECs, initiating alveolar regeneration (Fig. 8k) ${ }^{29,33,34}$.

\section{DISCUSSION}

Here, the centrality of platelet-derived SDF-1 in inducing alveolar regeneration was revealed by 'platelet-specific' gain and loss of function studies: first, regenerative alveolarization was impaired in thrombocytopenic mice caused by TPO knockout $\left(\mathrm{Thpo}^{-/-}\right)$ and pharmacological platelet depletion (Fig. 1); second, plateletspecific and inducible genetic ablation of the $S d f 1$ gene abrogated lung alveolar regeneration (Figs 2 and 3); third, intravascular transfusion of $S d f 1^{+/+}$platelets, but not $S d f 1^{-/-}$platelets, rescued the defective alveolarization of $\mathrm{Thpo}^{-/-}$mice (Figs 4 and 5); last, using an EC-specific gene deletion strategy $\left(M m p 14^{\mathrm{i} \Delta \mathrm{EC} / \mathrm{i} \Delta \mathrm{EC}}\right.$ and $C x c r 4^{\mathrm{i} \triangle \mathrm{EC} / \mathrm{i} \Delta \mathrm{EC}} C x c r 7^{\mathrm{i} \Delta \mathrm{EC} / \mathrm{i} \Delta \mathrm{EC}}$ ), we demonstrate that this rescue effect of SDF-1 platelets is mediated through CXCR4/7-dependent deployment of the pro-regenerative MMP14 pathway in the PCEC membrane (Figs 6-8). Hence, we propose that PNX recruits SDF$1^{+}$platelets to prime PCECs in the remaining lungs, constituting a haemo-vascular niche to orchestrate functional restoration of pulmonary tissues.

How the loss of the left lung recruits and activates platelets in the right lung remains to be studied. It is plausible that, after PNX, increased blood flow and circumferential shear force in the right lung activate platelets to adhere to pulmonary capillaries ${ }^{31,32,35,36,57}$. Alternatively, perturbation of biomechanical forces within the remaining lung activates pulmonary capillaries, thereby recruiting and activating circulating platelets ${ }^{42}$. In addition, how activated platelets influence other types of lung progenitor cell needs to be investigated in the future ${ }^{11-14,58}$.

Of note, the morphology of AEC1s is compromised when regeneration fails. After PNX, loss of the left lung lobe caused significantly increased mechanical stretch in the remaining lungs, possibly stimulating the matrix remodelling process ${ }^{6}$. Conceivably, in WT mice with normal regenerative capacity, this remodelling process coordinately accompanies the expansion of AEC1s. By contrast, when regeneration/cellular propagation fails in platelet-depleted mice or mice with a defective vascular niche, the alveolus undergoes remodelling in the absence of concomitant expansion of AECs. As such, in these mutant or antibody-treated mice, discordance between alveolar remodelling and lack of concurrent AEC propagation not only prevented the growth of new alveoli but also disrupted the morphology of existent AECs, compromising alveolar architecture.

Here we have used cell-type-specific 'loss and gain of function' studies to demonstrate that recruited platelets release SDF-1 to activate CXCR4/CXCR7 on PCECs in the early phase after PNX (days 1-5), eliciting cellular propagation and alveolarization that peaks at day 7 . It is likely that platelets represent a subpopulation of bone-marrowderived cells recruited into the lungs of pneumonectomized mice ${ }^{49}$. Whether bone-marrow-derived cells facilitate the pulmonary recruitment of activated platelets after PNX remains to be investigated. Furthermore, SDF-1 from other niche cells $s^{50-53}$ could play a prominent role in modulating tissue repair in other injury models.

We have also reconciled the mechanistic link between the previously demonstrated VEGFR2/FGFR1 pathways ${ }^{1,59}$ and the presented endothelial SDF-1-CXCR4/7 interaction ${ }^{24}$ in the study. SDF-1-CXCR4 signalling is regulated by the VEGFR2/FGFR1 pathways in PCECs after PNX, and Thpo-l- mice also exhibited blocked VEGFR2/FGFR1 signalling in PCECs. These findings imply that platelets also contribute to activation of the VEGFR2/FGFR1 pathways, which further potentiates SDF-1 signalling in PCECs. It is likely that activation of both pathways in PCECs leads to MMP14-dependent lung alveolarization, which is initially stimulated by platelets after PNX.

Lung disease remains the worldwide leading cause of death for adults $6,75,60-63$. Damage to the lungs after trauma or chemical injury results in initiation of reparative pathways. However, in most instances lung repair is insufficient to overcome the loss of functional parenchymal tissues ${ }^{13,64-66}$. Our finding implies that restoration of functional lung mass could be elicited in adult mammals by a coordinated interaction between alveolar progenitor cells and pro-regenerative cues from the vascular niche. As such, properly stimulating the cellular crosstalk between alveolar progenitor cells with the surrounding niche $e^{5,9-14,67,68}$ could instigate regeneration in adult human lungs with limited regenerative capacity, implicating the translational value of our study in combating chronic destructive lung diseases.

Uncovering the effects of platelet-derived SDF-1 in inducing lung regeneration holds further clinical implications. First, it is conceivable that thrombocytopenic patients will exhibit impaired regenerative ability after lung injury and show poor prognosis. To this end, periodic administration of SDF-1 or EGF to thrombocytopenic patients (for example, in an intensive care unit) could stimulate functional lung repair. Furthermore, the finding that infusion of SDF- $1^{+}$platelets initiates and sustains functional alveolarization suggests that transplantation of properly primed platelets represents an effective cell therapy approach to enable lung regeneration and repair.

Taken together, we have revealed a paradigm that loss of the left lung lobe recruits platelets to deploy SDF-1, preconditioning PCECs and eliciting lung alveolar regeneration. Infusion of functional platelets rescued the impaired lung regeneration by re-establishing a pro-regenerative haemo-vascular niche. Unravelling the cellular and molecular basis whereby platelets orchestrate lung regeneration permits a clinical strategy to initiate regenerative approach for lung diseases. 


\section{METHODS}

Methods and any associated references are available in the online version of the paper.

Note: Supplementary Information is available in the online version of the paper

\section{ACKNOWLEDGEMENTS}

We are grateful to T. Hla, R. Nachman and A. Choi (Weill Cornell) and S. Albelda and D. Cines (University of Pennsylvania) for critically evaluating our manuscript. $\mathrm{Thpo}^{-/-}$mice were provided by F. de Sauvage at Genentech. We would also like to thank R. H. Adams, S. J. Weiss and Y-R. Zou for offering inducible ECspecific Cdh5-(PAC)-Cre $e^{\mathrm{ERT} 2}$ and floxed Mmp14 and Cxcr4 mouse lines. We are indebted to M. E. Penfold at Chemocentryx for his help in providing floxed $\mathrm{Cxcr} 7$ mice. B-S.D. is supported by a National Scientist Development Grant from the American Heart Association (number 12SDG1213004). B-S.D. and Z.C. are both supported by Druckenmiller Fellowships from the New York Stem Cell Foundation. S.Y.R. is supported by Biotime. S.R. and K.S. are supported by Ansary Stem Cell Institute, the Empire State Stem Cell Board and New York State Department of Health grants (C026878, C028117, C029156), and S.R. is supported by the National Heart, Lung, and Blood Institute R01HL097797, R01HL115128, R01HL119872 and R01HL128158, National Cancer Institute U54CA163167 and the National Institute of Diabetes and Digestive and Kidney Diseases R01DK095039.

\section{AUTHOR CONTRIBUTIONS}

S.R. and Z.C. designed the experiments and wrote the paper. S.R., Z.C., R.L., I.I.S. and D.C. carried out the experiments and analysed the data. K.S. and S.Y.R. interpreted the data. B-S.D. conceived the project, carried out the experiments, analysed the data and wrote the paper. All authors commented on the manuscript.

\section{COMPETING FINANCIAL INTERESTS}

The authors declare no competing financial interests.

Published online at www.nature.com/doifinder/10.1038/ncb3096

Reprints and permissions information is available online at www.nature.com/reprints

1. Ding, B. S. et al. Endothelial-derived angiocrine signals induce and sustain regenerative lung alveolarization. Cell 147, 539-553 (2011).

2. Butler, J. P. et al. Evidence for adult lung growth in humans. N. Engl. J. Med. 367, 244-247 (2012)

3. Chen, L. Acciani, T., Le Cras, T, Lutzko, C. \& Perl, A. K. Dynamic regulation of platelet-derived growth factor receptor alpha expression in alveolar fibroblasts during realveolarization. Am. J. Respir. Cell Mol. Biol. 47, 517-527 (2012).

4. Thane, K., Ingenito, E. P. \& Hoffman, A. M. Lung regeneration and translational implications of the postpneumonectomy model. J. Lab. Clin. Med. 163, 363-376 (2014)

5. Rock, J. R. \& Hogan, B. L. Epithelial progenitor cells in lung development, maintenance, repair, and disease. Annu. Rev. Cell. Dev. Biol. 27, 493-512 (2011).

6. Beers, M. F. \& Morrisey, E. E. The three R's of lung health and disease: repair, remodeling, and regeneration. J. Clin. Invest. 121, 2065-2073 (2011).

7. Kotton, D. N. \& Morrisey, E. E. Lung regeneration: mechanisms, applications and emerging stem cell populations. Nat. Med. 20, 822-832 (2014).

8. Fehrenbach, H. et al. Neoalveolarisation contributes to compensatory lung growth following pneumonectomy in mice. Eur. Respir. J. 31, 515-522 (2008).

9. Barkauskas, C. E. et al. Type 2 alveolar cells are stem cells in adult lung. J. Clin. Invest. 123, 3025-3036 (2013).

10. Desai, T. J., Brownfield, D. G. \& Krasnow, M. A. Alveolar progenitor and stem cells in lung development, renewal and cancer. Nature 507, 190-194 (2014).

11. Kumar, P. A. et al. Distal airway stem cells yield alveoli in vitro and during lung regeneration following H1N1 influenza infection. Cell 147, 525-538 (2011).

12. Chapman, H. A. et al. Integrin $\alpha 6 \beta 4$ identifies an adult distal lung epithelial population with regenerative potential in mice. J. Clin. Invest. 121, 2855-2862 (2011).

13. Stripp, B. R. \& Shapiro, S. D. Stem cells in lung disease, repair, and the potential for therapeutic interventions: state-of-the-art and future challenges. Am. J. Respir. Cell Mol. Biol. 34, 517-518 (2006).

14. Lee, J. H. et al. Lung stem cell differentiation in mice directed by endothelial cells via a BMP4-NFATc1-thrombospondin-1 axis. Cell 156, 440-455 (2014).

15. Petrache, I. et al. Ceramide upregulation causes pulmonary cell apoptosis and emphysema-like disease in mice. Nat. Med. 11, 491-498 (2005).

16. Seimetz, M. et al. Inducible NOS inhibition reverses tobacco-smoke-induced emphysema and pulmonary hypertension in mice. Cell 147, 293-305 (2011).

17. White, A. C., Lavine, K. J. \& Ornitz, D. M. FGF9 and SHH regulate mesenchymal Vegfa expression and development of the pulmonary capillary network. Development 134, 3743-3752 (2007)

18. Lazarus, A. et al. A perfusion-independent role of blood vessels in determining branching stereotypy of lung airways. Development 138, 2359-2368 (2011).
19. Red-Horse, K., Crawford, Y., Shojaei, F. \& Ferrara, N. Endothelium-microenvironment interactions in the developing embryo and in the adult. Dev. cell 12 181-194 (2007).

20. Chun, T. H. et al. A pericellular collagenase directs the 3-dimensional development of white adipose tissue. Cell 125, 577-591 (2006).

21. Tang, Y. et al. MT1-MMP-dependent control of skeletal stem cell commitment via a $\beta 1$-integrin/YAP/TAZ signaling axis. Dev. cell 25, 402-416 (2013).

22. Stratman, A. N. et al. Endothelial cell lumen and vascular guidance tunnel formation requires MT1-MMP-dependent proteolysis in 3-dimensional collagen matrices. Blood 114, 237-247 (2009).

23. Koziol, A. et al. The protease MT1-MMP drives a combinatorial proteolytic program in activated endothelial cells. FASEB J. 26, 4481-4494 (2012).

24. Ding, B. S. et al. Divergent angiocrine signals from vascular niche balance liver regeneration and fibrosis. Nature 505, 97-102 (2014).

25. Ding, B. S. et al. Inductive angiocrine signals from sinusoidal endothelium are required for liver regeneration. Nature 468, 310-315 (2010).

26. Weyrich, A. S. \& Zimmerman, G. A. Platelets in lung biology. Annu. Rev. Physiol. 75 , 569-591 (2013).

27. Fuentes, R. et al. Infusion of mature megakaryocytes into mice yields functional platelets. J. Clin. Invest. 120, 3917-3922 (2010).

28. Lesurtel, M. et al. Platelet-derived serotonin mediates liver regeneration. Science 312, 104-107 (2006)

29. Bertozzi, C. C., Hess, P. R. \& Kahn, M. L. Platelets: covert regulators of lymphatic development. Arterioscler. Thromb. Vasc. Biol. 30, 2368-2371 (2010).

30. Min, S. H. et al. Loss of PIKfyve in platelets causes a lysosomal disease leading to inflammation and thrombosis in mice. Nat. Commun. 5, 4691 (2014).

31. Bennett, J. S., Berger, B. W. \& Billings, P. C. The structure and function of platelet integrins. J. Thromb. Haemost. 7, 200-205 (2009).

32. Herzog, B. H. et al. Podoplanin maintains high endothelial venule integrity by interacting with platelet CLEC-2. Nature 502, 105-109 (2013).

33. Gleissner, C. A., von Hundelshausen, P. \& Ley, K. Platelet chemokines in vascular disease. Arterioscler. Thromb. Vasc. Biol. 28, 1920-1927 (2008).

34. Jin, D. K. et al. Cytokine-mediated deployment of SDF-1 induces revascularization through recruitment of CXCR4+ hemangiocytes. Nat. Med. 12, 557-567 (2006).

35. Bruns, I. et al. Megakaryocytes regulate hematopoietic stem cell quiescence through CXCL4 secretion. Nat. Med. 20, 1315-1320 (2014).

36. Shen, B. et al. A directional switch of integrin signalling and a new anti-thrombotic strategy. Nature 503, 131-135 (2013).

37. Zhao, M. et al. Megakaryocytes maintain homeostatic quiescence and promote postinjury regeneration of hematopoietic stem cells. Nat. Med. 20, 1321-1326 (2014).

38. Sreeramkumar, V. et al. Neutrophils scan for activated platelets to initiate inflammation. Science 346, 1234-1238 (2014).

39. Schumacher, D., Strilic, B., Sivaraj, K. K., Wettschureck, N. \& Offermanns, S. Platelet-derived nucleotides promote tumor-cell transendothelial migration and metastasis via P2Y2 receptor. Cancer Cell 24, 130-137 (2013).

40. Massberg, S. et al. Platelets secrete stromal cell-derived factor $1 \alpha$ and recruit bone marrow-derived progenitor cells to arterial thrombi in vivo. J. Exp. Med. $\mathbf{2 0 3}$ 1221-1233 (2006)

41. Boilard, E. et al. Platelets amplify inflammation in arthritis via collagen-dependent microparticle production. Science 327, 580-583 (2010).

42. Frenette, P. S., Johnson, R. C., Hynes, R. O. \& Wagner, D. D. Platelets roll on stimulated endothelium in vivo: an interaction mediated by endothelial P-selectin. Proc. Natl Acad. Sci. USA 92, 7450-7454 (1995).

43. Clark, S. R. et al. Platelet TLR4 activates neutrophil extracellular traps to ensnare bacteria in septic blood. Nat. Med. 13, 463-469 (2007).

44. Caudrillier, A. et al. Platelets induce neutrophil extracellular traps in transfusionrelated acute lung injury. J. Clin. Invest. 122, 2661-2671 (2012).

45. Meyer, A. et al. Platelet TGF- $\beta 1$ contributions to plasma TGF- $\beta 1$, cardiac fibrosis, and systolic dysfunction in a mouse model of pressure overload. Blood 119, 1064-1074 (2012).

46. Labelle, M., Begum, S. \& Hynes, R. O. Direct signaling between platelets and cancer cells induces an epithelial-mesenchymal-like transition and promotes metastasis. Cancer Cell 20, 576-590 (2011).

47. Dar, A. et al. Chemokine receptor CXCR4-dependent internalization and resecretion of functional chemokine SDF-1 by bone marrow endothelial and stromal cells. Nat. Immunol. 6, 1038-1046 (2005).

48. Tachibana, K. et al. The chemokine receptor CXCR4 is essential for vascularization of the gastrointestinal tract. Nature 393, 591-594 (1998).

49. Seeger, F. H. et al. Heparin disrupts the CXCR4/SDF-1 axis and impairs the functional capacity of bone marrow-derived mononuclear cells used for cardiovascular repair. Circ. Res. 111, 854-862 (2012)

50. Omatsu, Y. et al. The essential functions of adipo-osteogenic progenitors as the hematopoietic stem and progenitor cell niche. Immunity 33, 387-399 (2010).

51. Li, W. et al. Peripheral nerve-derived CXCL12 and VEGF-A regulate the patterning of arterial vessel branching in developing limb skin. Dev. cell 24, 359-371 (2013)

52. Ding, L. \& Morrison, S. J. Haematopoietic stem cells and early lymphoid progenitors occupy distinct bone marrow niches. Nature 495, 231-235 (2013).

53. Greenbaum, A. et al. CXCL12 in early mesenchymal progenitors is required for haematopoietic stem-cell maintenance. Nature 495, 227-230 (2013). 
54. Potente, M., Gerhardt, H. \& Carmeliet, P. Basic and therapeutic aspects of angiogenesis. Cell 146, 873-887 (2011).

55. Ding, B. S. et al. Lung endothelium targeting for pulmonary embolism thrombolysis Circulation 108, 2892-2898 (2003).

56. Wang, Y. et al. Ephrin-B2 controls VEGF-induced angiogenesis and lymphangiogenesis. Nature 465, 483-486 (2010).

57. Brass, L. F., Zhu, L. \& Stalker, T. J. Minding the gaps to promote thrombus growth and stability. J. Clin. Invest. 115, 3385-3392 (2005).

58. Tata, P. R. et al. Dedifferentiation of committed epithelial cells into stem cells in vivo. Nature 503, 218-223 (2013)

59. Cao, Z. et al. Angiocrine factors deployed by tumor vascular niche induce $\mathrm{B}$ cell lymphoma invasiveness and chemoresistance. Cancer Cell 25, 350-365 (2014).

60. Hecker, L. et al. NADPH oxidase-4 mediates myofibroblast activation and fibrogenic responses to lung injury. Nat. Med. 15, 1077-1081 (2009).

61. Henderson, N. C. et al. Targeting of $\alpha_{v}$ integrin identifies a core molecular pathway that regulates fibrosis in several organs. Nat. Med. 19, 1617-1624 (2013).
62. Islam, M. N. et al. Mitochondrial transfer from bone-marrow-derived stromal cells to pulmonary alveoli protects against acute lung injury. Nat. Med. 18, 759-765 (2012).

63. Jiang, D. et al. Regulation of lung injury and repair by Toll-like receptors and hyaluronan. Nat. Med. 11, 1173-1179 (2005).

64. Gotts, J. E. \& Matthay, M. A. Cell therapy for lung disease: a step forward. Ches 143, 1525-1527 (2013).

65. Duffield, J. S., Lupher, M., Thannickal, V. J. \& Wynn, T. A. Host responses in tissue repair and fibrosis. Annu. Rev. Pathol. 8, 241-276 (2013).

66. Tager, A. M. et al. The lysophosphatidic acid receptor LPA1 links pulmonary fibrosis to lung injury by mediating fibroblast recruitment and vascular leak. Nat. Med. 14 45-54 (2008)

67. Martinu, T., Palmer, S. M. \& Ortiz, L. A. Lung-resident mesenchymal stromal cells. A new player in post-transplant bronchiolitis obliterans syndrome? Am. J. Respir. Crit. Care Med. 183, 968-970 (2011).

68. Forbes, S. J. \& Rosenthal, N. Preparing the ground for tissue regeneration: from mechanism to therapy. Nat. Med. 20, 857-869 (2014). 


\section{METHODS}

Animals. The Cdh5(PAC)-Cre $e^{\mathrm{ERT2}}$ (VE-cadherin-Cre ${ }^{\mathrm{ERT} 2}$ ) mouse line was provided by R. Adams ${ }^{56}, R o s a-C r e^{\mathrm{ERT} 2}$ animals expressing tamoxifen-responsive inducible $\mathrm{Cre}^{\mathrm{ERT2}}$, loxP-site-flanked $\mathrm{Cxcr} 4$ and $\mathrm{Cxcr} 7$ mice and $\mathrm{Thpo}^{-/-}$mice have been described previously ${ }^{1,25,34}$. Floxed Mmp14 mice were offered by S. Weiss at the University of Michigan ${ }^{21}$. C57/B6, Pf4-Cre, floxed Sdf1 (ref. 53) and actin-tdTomato mice ubiquitously expressing tdTomato fluorescent protein $(\beta$-actin promoterdriven tdTomato) were obtained from Jackson Laboratory. To activate Cdh5(PAC)$\mathrm{Cr}^{\mathrm{ERT} 2}$ and $\mathrm{Ros} a-\mathrm{Cr} e^{\mathrm{ERT} 2}$, mice were treated with tamoxifen six times at dose of $150 \mathrm{mg} \mathrm{kg}^{-1}$. After daily injection of tamoxifen for three consecutive days, mice were rested for three days and then treated with tamoxifen for another three days. Mice were used for surgery or analysis at least 30 days after final tamoxifen treatment. To compare phenotypes between different genotypes, age/sex/weight-matched littermates were used in corresponding experimental groups. Mice at $20-28 \mathrm{~g}$ bodyweight were used. Investigators who established the mouse lung regeneration model and analysed the pattern and extent of cell activation/proliferation were randomly assigned with animals or samples from different experimental groups and were blind to the genotypes of individual groups. All animal procedures were under the guidance of the NIH and approved by IACUC at Weill Cornell. Chemicals were from Sigma unless otherwise specified.

Left lung unilateral pneumonectomy model and measurement of alveolar regeneration. The PNX procedure was adapted as described ${ }^{1}$. Mice were anaesthetized with $100 \mathrm{mg} \mathrm{kg}^{-1}$ intraperitoneal ketamine and $10 \mathrm{mg} \mathrm{kg}^{-1}$ xylazine. Orotracheal intubation was carried out in anaesthetized and mechanically ventilated mice. The left lung lobe was resected with a suture tied around the hilum. Shamoperated mice were subjected to thoracotomy without lobe resection. To determine the role of recombinant EGF in alveolar regeneration, mice were injected with $500 \mu \mathrm{g} \mathrm{kg}^{-1}$ recombinant mouse EGF (Abcam) on a daily basis after PNX.

Immunostaining and morphometric analysis. Each parameter from each individual animal was measured at least twice and averaged. Before killing, mice were anaesthetized, blood was obtained through the inferior vena cava and the remaining right lung lobes received an intratracheal instillation of reconstituted optimal cutting temperature compound (Tissue-Tek) to a pressure of $25 \mathrm{~cm} \mathrm{H}_{2} \mathrm{O}$. The trachea was then tied under pressure, and the lungs were cryoprotected and snap frozen in optimal cutting temperature compound. For immunofluorescence microscopy, the tissue sections $(10 \mu \mathrm{m})$ were blocked with $5 \%$ normal donkey serum $/ 1 \%$ bovine serum albumin/0.1\% Triton X-100 and incubated in primary antibodies. PCECs were identified by staining of anti-VE-cadherin polyclonal antibodies (R\&D Systems AF1002, $2 \mu \mathrm{g} \mathrm{ml}^{-1}$ ); AEC1s and AEC2s were determined by staining with podoplanin (R\&D Systems, AF3244, $2 \mu \mathrm{g} \mathrm{ml}^{-1}$ ) and aquaporin 5 (Abcam, ab78486, $2 \mu \mathrm{g} \mathrm{ml}^{-1}$ ) for AEC1s and SFTPC (Millipore, Ab3786, $2 \mu \mathrm{g} \mathrm{ml}^{-1}$ ) for AEC2s. Platelet deposition was detected using anti-CD41 mAb (MWReg30, BD Biosciences, $\left.1 \mu \mathrm{g} \mathrm{ml}^{-1}\right)$. After incubation in fluorophore-conjugated secondary antibodies $\left(2.5 \mu \mathrm{g} \mathrm{ml}^{-1}\right.$, Jackson ImmunoResearch), nuclear counterstaining was carried out with DAPI (Invitrogen). No appreciable staining was observed in isotype IgG controls. AEC2s and PCECs were quantified by staining with antibodies recognizing SFTPC and VE-cadherin, respectively. Fluorescent images were captured on an AxioVert LSM710 microscope (Zeiss). For image analysis, staining signals in each slide (such as SFTPC, VE-cadherin, podoplanin, aquaporin 5 and CD41) were independently evaluated and quantified by two investigators from five randomly selected fields of view. Analysis of the image was carried out blind. Investigators were not aware of the genotype of animals or identity of samples during scoring.

Lung cell proliferation in vivo was measured by BrdU uptake. Mice received a single intraperitoneal injection of BrdU (Sigma; at a dose of $50 \mathrm{mg}$ per $\mathrm{kg}$ animal weight) $60 \mathrm{~min}$ before killing, and the incorporation of BrdU was measured by immunostaining on cryosections and flow cytometry as previously described $^{1,51}$. Cryosections were stained using the BrdU Detection System (BD Biosciences) and fluorophore-conjugated secondary antibodies $\left(2.5 \mu \mathrm{g} \mathrm{ml}^{-1}\right.$, Jackson ImmunoResearch) $)^{1,51}$. The extent of BrdU incorporation was first determined in sham-operated mice of all used genotypes. To assess the difference in cell proliferation after $\mathrm{PNX}$, the percentage of $\mathrm{BrdU}^{+} \mathrm{VE}$-cadherin ${ }^{+}$proliferating PCECs and $\mathrm{BrdU}^{+}$SFTPC $^{+}$AEC2s in both control and mutant groups were compared at day 7 .

Measurement of functional alveologenesis after PNX. Lung respiratory function parameters, including inspiratory capacity and lung parenchymal tissue compliance, were measured using the forced oscillation technique operated by flexiWare 7 software in a computer-controlled piston ventilator (SCIREQ). Inspiratory capacity was measured during a recruitment manoeuvre using the above software. Oxygen tension in the arterial blood was measured as previously described using I-Stat ${ }^{69}$ (Abbott Laboratories).
H\&E staining was carried out on retrieved lung tissues to evaluate alveologenesis after PNX. Alveolar structure in each H\&E slide was independently quantified by two investigators from five random fields. Mean linear interception $\left(L_{\mathrm{m}}\right)$ was measured as previously described ${ }^{70}$. Briefly, 10 equally distributed horizontal lines and 11 evenly distributed vertical lines were drawn over an H\&E-stained section. For each line, the intercepts with the alveolar tissue are counted. $L_{\mathrm{m}}$ is calculated as the averaged ratio between line length and the number of intercepts placed on the lung section.

Alveolar number was determined as previously described ${ }^{8}$. Briefly, the lungs were cut into $3 \mathrm{~mm}$ thick lung slices and subsequent cutting of $3 \mathrm{~mm}$ wide bars with randomized tissue orientation. Embedded bars were cut into $20 \mu \mathrm{m}$ thick serial sections. Sections were collected on slides and subjected to H\&E staining for determination of the mean bar thickness. The block was then cut to a series of $5 \mu \mathrm{m}$ thick serial sections and collected on slides. The ratio of serial section thickness/mean bar thickness was then determined. To obtain a slide for alveolar number determination, a series of sections for each tissue block was systematically scanned along the $x$ - and $y$-axes to yield a uniform, random sample with disector pairs of $600 \mu \mathrm{m} \times 600 \mu \mathrm{m}$ sections. Then the number of alveoli was measured by assessing the Euler number $(x)$ of the network of alveolar openings within a counting frame. For measurement of the Euler number, all alveolar openings in the examined section were counted in each frame. To increase the efficiency of alveolar number measurement, both directions in the frame were measured in each individual field of view. Then the total number of alveoli per lung was calculated based on alveolar number within the area of the counting frame and the disector pair size on the slide, as well as the ratio between serial section thickness and mean bar thickness.

Isolation and analysis of mouse PCECs. Isolation of PCECs and examination of phosphorylation and total protein levels of Akt, VEGFR2, FRS2, CXCR4 and MMP14 were carried out as described ${ }^{1,51}$. Briefly, the lungs was perfused at $5 \mathrm{ml} \mathrm{min}^{-1}$ through the pulmonary artery with Liver Perfusion Medium (Invitrogen) at $37^{\circ} \mathrm{C}$ for $10 \mathrm{~min}$, followed by perfusion with Liver Digest Medium (Invitrogen) for another $10 \mathrm{~min} .1 \mathrm{ml}$ digestion medium was also injected into the alveolar space through the exposed trachea. The lung tissue was then dissociated in Hepatocyte Wash medium (Invitrogen) and passed through Dacron fabric with $70 \mu \mathrm{m}$ pores, and the flowthrough containing PCECs was collected, washed twice and pelleted at $350 \mathrm{~g}$ for $7 \mathrm{~min}$. Fractions containing PCECs were enriched, washed with an equal volume of PBS and centrifuged at $900 \mathrm{~g}$ for $7 \mathrm{~min}$. The cell pellet was then washed with PBS at $350 \mathrm{~g}$ for $7 \mathrm{~min}$ and incubated with Dynabeads magnetic beads (Invitrogen) coated with rat anti-mouse CD31 and intercellular cell adhesion molecule (ICAM)-2 antibodies (MEC13.3, BD Biosciences). The purification of $\mathrm{CD} 31^{+} \mathrm{ICAM}^{+}$PCECs was carried out following the manufacturer's protocol. To maintain protein phosphorylation in the isolated PCECs, mouse lungs were perfused through the pulmonary artery with phosphatase inhibitor cocktails (Sigma) after mice were killed, and whole processing was carried out in the presence of phosphatase inhibitor cocktails.

Isolated platelets or cultured PCECs were lysed with RIPA (radioimmunoprecipitation assay) buffer: $1 \times$ tris-buffered saline, $1 \%(\mathrm{v} / \mathrm{v})$ Nonidet P-40, $0.5 \%(\mathrm{w} / \mathrm{v})$ sodium deoxycholate, $0.1 \%(\mathrm{v} / \mathrm{v})$ SDS, $1 \mathrm{mM}$ sodium orthovanadate, $10 \mathrm{mM} \mathrm{NaF}$ and $1 \times$ protease inhibitor cocktail (Roche). Phosphorylation and protein levels of VEGFR2 and the FGFR1 downstream effector FRS2 in PCECs were determined as described ${ }^{51}$.

Equal amounts of protein were subjected to SDS-polyacrylamide gel electrophoresis in $4-12 \%$ Bis-Tris gels (Invitrogen). Proteins were transferred to nitrocellulose membranes, and underwent immunoblotting with antibodies against VE-cadherin (R\&D, AF1002, $1 \mu \mathrm{g} \mathrm{ml}^{-1}$ ), VEGFR2 (Cell Signaling, 2472, $1 \mu \mathrm{g} \mathrm{ml}^{-1}$ ), phosphorylated VEGFR2 (Cell Signaling, 3817, $1 \mu \mathrm{g} \mathrm{m}^{-1}$ ), total AKT (Cell Signaling, 9272, $1 \mu \mathrm{g} \mathrm{ml}^{-1}$ ), phosphorylated Akt (Cell Signaling, 2965, $1 \mu \mathrm{g} \mathrm{ml}^{-1}$ ), total FRS2 (Abcam, ab137458, $1 \mu \mathrm{g} \mathrm{ml}^{-1}$ ), total CXCR4 (Abcam, ab2074, $2 \mu \mathrm{g} \mathrm{ml}^{-1}$ ), total MMP14 (Abcam, ab51074 and ab53712, $1 \mu \mathrm{g} \mathrm{ml}^{-1}$ ) and SDF-1 (Cell Signaling, $3740,2 \mu \mathrm{g} \mathrm{ml}^{-1}$ ). $\beta$-actin (Santa Cruz, sc-1616, $2 \mu \mathrm{g} \mathrm{ml}^{-1}$ ) in the loaded sample was also tested to control for the loaded protein amount.

To test the protein components in the alveolar space, BALF was obtained from sham or pneumonectomized mice by lavaging $1 \mathrm{ml}$ of $\mathrm{PBS}$ through the trachea, and retrieved BALF was concentrated to $100 \mu \mathrm{l}$ with a protein concentration column (Millipore). The HB-EGF protein level in BALF was assessed as previously described $^{1}$, using rabbit polyclonal antibody from Santa Cruz.

Depletion of circulating platelets; stimulation of thrombopoiesis. To stimulate thrombopoiesis, recombinant TPO was intraperitoneally injected into $\mathrm{Thpo}^{-/-}$or WT mice at a dose of $25 \mathrm{\mu g} \mathrm{kg}^{-1}$ on a daily basis 10 days before PNX and afterwards. To deplete circulating platelets, rat anti-mouse CD41 mAb (BD Biosciences, clone MWReg30) was intraperitoneally injected into mice at a dose of $10 \mathrm{mg} \mathrm{kg}^{-1}$ every 3 days. Recombinant TPO, EGF and SDF-1 were obtained from PeproTech. Vehicle for individual cytokines was also injected as a control group. 
The effects of injected cytokine on alveolar regeneration were evaluated with control groups treated with vehicle, including alteration in circulating platelets and parameters of alveologenesis (as described above). In particular, mouse platelets were collected at indicated times from the retrobulbar venous plexus of anaesthetized mice, using heparinized haematocrit tubing. The platelet count in peripheral blood was then determined with an automated Advia 120 Hematology System (Siemens Healthcare).

Isolation and stimulation of mouse platelets and intravascular infusion of platelets into mice. To maximize the yield of platelets, the blood remaining from this first centrifugation was diluted with PBS to the original volume and recentrifuged. Mouse platelets isolated by centrifugation were treated with agonist ADP $(5$ or $10 \mu \mathrm{M})$ or $0.1 \mathrm{U} \mathrm{ml}^{-1}$ thrombin. After agonist addition, the reaction was terminated and incubation microfuge tubes were centrifuged $(1 \mathrm{~min}, 14,000 \mathrm{~g})$. The supernatant of the stimulated platelets was then aliquoted for activation of primary PCECs.

The strategy to infuse platelets through the jugular vein was adopted from previous work ${ }^{49}$. Platelets were isolated and concentrated from mice with an inducible deletion of $S d f 1$ ( $S d f 1^{\Delta / \Delta}$ mice) to obtain $S d f 1^{-/-}$platelets. Platelets from their WT littermates, or $\beta$-actin promoter-driven tdTomato reporter mice, were used as $S d f 1^{+/+}$platelets. After isolation, $2 \times 10^{9} \mathrm{Sdf1}^{+/+}$or $S d f 1^{-/-}$platelets were infused through the exposed jugular vein of mice over a period of $15 \mathrm{~min}$. This injection route yielded the highest accumulation of platelets in the pulmonary vasculature of recipient mice. To track the localization of infused $\mathrm{CD} 41^{+}$tdTomato $^{+}$ platelets in the recipient mouse lungs, total cells were isolated from the recipient and analysed for surface expression of tdTomato fluorescent signal and CD41. Immunofluorescent staining of CD41 and VE-cadherin was also carried out to examine the association of PCECs with transplanted platelets, as described in 'Immunostaining and morphometric analysis'.

Cultivation of mouse and human PCECs and gene knockdown. Primary mouse PCECs were obtained from Angiocrine Bioscience (New York) and cultured as previously described ${ }^{1}$. Human PCECs were from ScienCell Research Laboratories and cultivated following the vendor's instructions. To knock down Cxcr4 and Cxcr7 selectively in PSECs, Cxcr4 and Cxcr7/scrambled shRNA lentiviruses were generated by co-transfecting $15 \mu \mathrm{g}$ of shuttle lentiviral vector containing Id1/scrambled shRNA, $3 \mu \mathrm{g}$ pENV/VSV-G, $5 \mu \mathrm{g}$ pRRE and $2.5 \mu \mathrm{g}$ pRSV-REV in $293 \mathrm{~T}$ cells using FuGENE 6 (Roche Applied Science). Viral supernatants were concentrated by ultracentrifugation. These concentrated viral preparations were used to transduce PCECs. Human CXCR4 was silenced by treatment with combined shRNA clones: TRCN0000004052, TRCN0000004053, TRCN0000004054 and TRCN0000004055 (Open Biosystems), and CXCR7 was knocked down with clone TL305345 (OriGene). Cxcr4 was knocked down in mouse PCECs by transduction with a combination of clones TRCN0000028678, TRCN0000028704 and TRCN0000028724. Cxcr7 was silenced in mouse PCECs with shRNA cloneTL515747 from Origene.

Supernatant from platelets was used to treat PCECs after starvation in minimal medium that consists of Medium 199 (HyClone) and 2\% BSA. Six hours after treatment with platelet supernatant or $10 \mathrm{ng} \mathrm{ml}^{-1}$ SDF-1, PCECs were retrieved for analysis of MMP14 protein level by immunoblot after cell lysis, immunostaining and flow cytometry. MMP14 was detected by rabbit polyclonal antibody from Abcam (ab51074 and ab53712, $1 \mu \mathrm{g} \mathrm{ml}^{-1}$ ).

Isolation of membrane protein of PCECs after pulmonary perfusion and in situ biotinylation. Biotinylation of PCEC membrane protein in mice was achieved by pulmonary perfusion through the pulmonary artery ${ }^{53}$. After the mouse pulmonary artery was exposed, a 25-gauge catheter was inserted and fastened. PBS was used to perfuse the mouse pulmonary vasculature. Then the pulmonary vasculature was perfused with PBS containing $10 \mathrm{mg} \mathrm{ml}^{-1}$ membraneimpermeable sulpho-NHS-LC-biotin that recognizes primary amine in protein (Pierce) at $1 \mathrm{ml} \mathrm{min}^{-1}$ for $10 \mathrm{~min}$. PCEC membrane proteins that are accessible to circulation were biotinylated. After unconjugated biotin was quenched by $20 \mathrm{mg}$ glycine, lung tissues were harvested and homogenized, and biotinylated proteins were isolated using $500 \mu \mathrm{lg}^{-1}$ tissue streptavidin beads (Pierce) and subjected to immunoblot against MMP14 and VE-cadherin after sonication and boiling. This in situ biotinylation strategy provides an approach to isolate and assay membrane fractions of lung ECs. The protein level of MMP14 in isolated membrane proteins after PNX was determined by immunoblot (Abcam), and the EC-specific marker VE-cadherin was also blotted (R\&D systems) as a control for the loading of endothelial membrane proteins. To control for the purity of isolated EC membrane proteins, endothelial membrane proteins such as platelet-endothelial cell adhesion molecule (PECAM)-1 (Santa Cruz Biotechnology, sc-1506, $10 \mu \mathrm{g} \mathrm{ml}^{-1}$ ), ICAM-1 (Santa Cruz Biotechnology, sc-1511, $10 \mu \mathrm{g} \mathrm{ml}^{-1}$ ), VEGFR2, angiotensin converting enzyme (sc-20998, $2 \mu \mathrm{g} \mathrm{ml}^{-1}$ ), integrin $\alpha \mathrm{v}$ (sc-6618, $\left.5 \mu \mathrm{g} \mathrm{ml}^{-1}\right), \alpha 5\left(\mathrm{sc}-10729,5 \mu \mathrm{g} \mathrm{ml}^{-1}\right)$, E-cadherin (sc-7870, $10 \mu \mathrm{g} \mathrm{m}^{-1}$ ) and cytoplasmic proteins $\alpha$-tubulin (sc-53646, $10 \mu \mathrm{g} \mathrm{ml}^{-1}$ ) and $\beta$ actin were also examined in the isolated membrane fractions by immunoblot as described above.

Mouse model of unilateral in situ lung ischaemia-reperfusion. Acute lung injury was also introduced by a mouse model of lung ischaemia-reperfusion (I/R). Unilateral left lung $\mathrm{I} / \mathrm{R}$ was created using a protocol previously described in detail ${ }^{69}$. Briefly, after carrying out a thoracotomy in ventilated anaesthetized mice, the hilum of the left lung was cross-clamped for $30 \mathrm{~min}$ followed by $120 \mathrm{~min}$ of reperfusion. Five minutes before killing, $400 \mathrm{U}$ of heparin was injected intravenously to prevent post-mortem clotting and the left lobe of the lung was excised. Sham-operated mice underwent the same procedure except that clamping of the hilum was omitted. Lung specimens were weighed and homogenized in PBS ( $\mathrm{pH}$ 7.2) containing protease inhibitor cocktail (Sigma), aprotinin $\left(30 \mathrm{KIU} \mathrm{ml}^{-1}\right)$, heparin $15 \mathrm{U} \mathrm{ml}^{-1}$ and $100 \mathrm{mM}$ 6-aminohexanoic acid. Aliquots of tissue homogenates were prepared for analysis. Lung fibrin was extracted from one aliquot and detected by western blot using fibrin $\beta$-chain-specific antibody 350 (American Diagnostica, $5 \mu \mathrm{g} \mathrm{ml}^{-1}$ ), as described previously ${ }^{69}$. Total lung proteins were extracted with $1 \%$ SDS and $1 \%$ Triton X100 and subjected to western blot using anti- $\beta$-actin. Lung fibrin was quantified by densitometric analysis and normalized to $\beta$-actin. Lung fibrin deposition in lungs was compared between different groups.

Flow cytometric analysis. To reveal platelet and PCEC activation, deposition and proliferation, total lung cells were isolated from perfused mouse lung tissues after dissociation with medium containing collagenase/dispase/DNase ${ }^{1}$. Retrieved lung cells were incubated with conjugated antibodies and analysed by flow cytometry on LSRII-SORP (BD; ref. 51). Data were processed with FACSDiva 6.1 software (BD). All doublets were ruled out by FSC-W $\times$ FSC-H and SSC-W $\times$ SSC-H analysis. Monoclonal antibodies were conjugated to Alexa Fluor dyes or Qdots according to manufacturers' protocols (Molecular Probes/Invitrogen), including VE-cadherin (clone BV13, ImClone, $5 \mu \mathrm{g} \mathrm{ml}^{-1}$ ), CD41 (clone MWReg30, BD Bioscience, $5 \mu \mathrm{g} \mathrm{ml}^{-1}$ ) and P-selectin (clone RB40.34, BD Bioscience, $5 \mu \mathrm{g} \mathrm{ml}^{-1}$ ). Single-stained channels were used for compensation. Flow cytometry analysis was carried out using various controls, including unstained cells, isotype antibodies and fluorophore minus one controls for determining gates and compensations in flow cytometry.

Statistical analysis. Experiments were repeated at least three times. We included all tested animals for quantification to analyse the statistical difference. A representative image from each animal group is presented in the figure. If higher variability was found, we increased the sample size to fully confirm statistical significance. No statistical method was used to predetermine sample size. Statistical analysis between individual experimental groups was determined by an unpaired two-tailed $t$-test. All data points from an individual animal or cell are presented in the format of a dot plot.

Reproducibility of experiments. All presented representative images were obtained from independently repeated experiments. Immunostaining images in Figs 1f,j,o, 2a,e,j, 3g,j, 4h,l, 5a,i, 6d,k,m,o and $7 \mathrm{~b}$ and Supplementary Figs 1a-d, $2 \mathrm{a}, \mathrm{c}, \mathrm{e}, 3 \mathrm{~g}, \mathrm{~h}, 5 \mathrm{a}$ and $7 \mathrm{a}$ were independently repeated three times. Immunoblot images in Figs 2i, 3b, 5b,d,e,g,h,l,m,n, 7k,m and 8b,e,h and Supplementary Figs 6a,c and $8 \mathrm{a}, \mathrm{b}$ were from four repeated experiments, and flow cytometry micrographs in Figs $1 \mathrm{~b}, \mathrm{~h}, \mathrm{n}, 2 \mathrm{~b}, 3 \mathrm{~h}, 4 \mathrm{i}, 6 \mathrm{f}, \mathrm{n}$ and $7 \mathrm{e}, \mathrm{h}$ and Supplementary Fig. $7 \mathrm{c}$ were repeated three times. Used animal numbers per groups are described in the respective figure legends.

69. Ding, B. S. et al. Anchoring fusion thrombomodulin to the endothelial lumen protects against injury-induced lung thrombosis and inflammation. Am. J. Respir. Crit. Care Med. 180, 247-256 (2009).

70. Sato, A. et al. Morphological mechanism of the development of pulmonary emphysema in klotho mice. Proc. Natl Acad. Sci. USA 104, 2361-2365 (2007). 
a

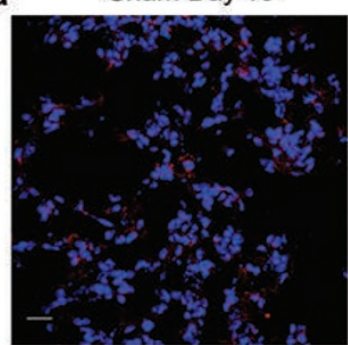

b

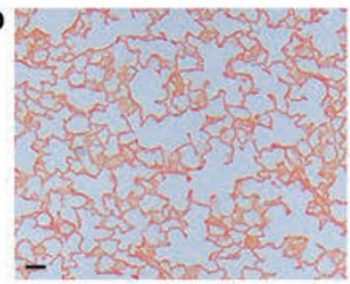

PNX Day 10
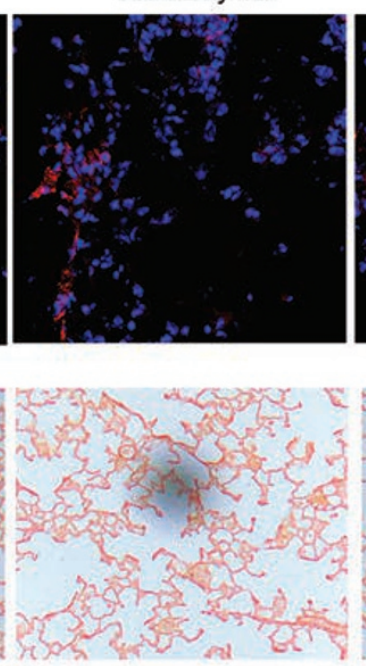

PNX Day 18
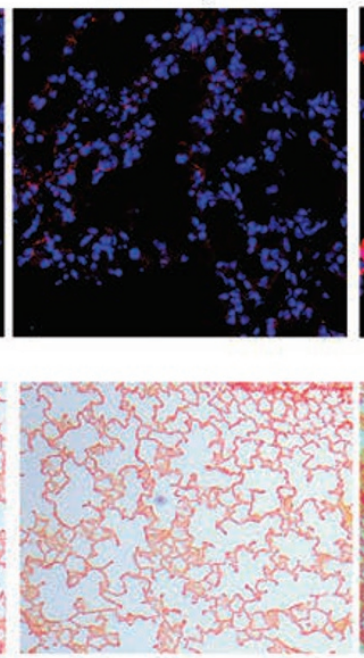

I.T. Bleo Day 21

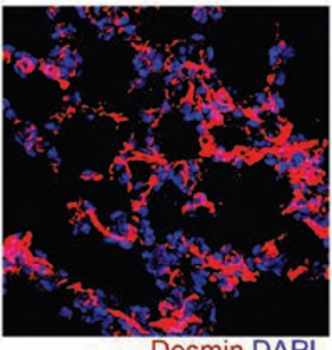

Desmin DAPI

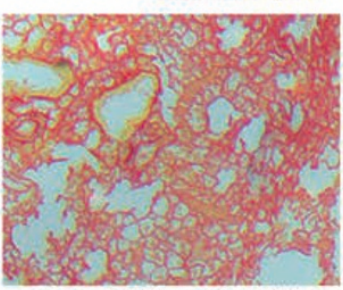

Sirius red staining

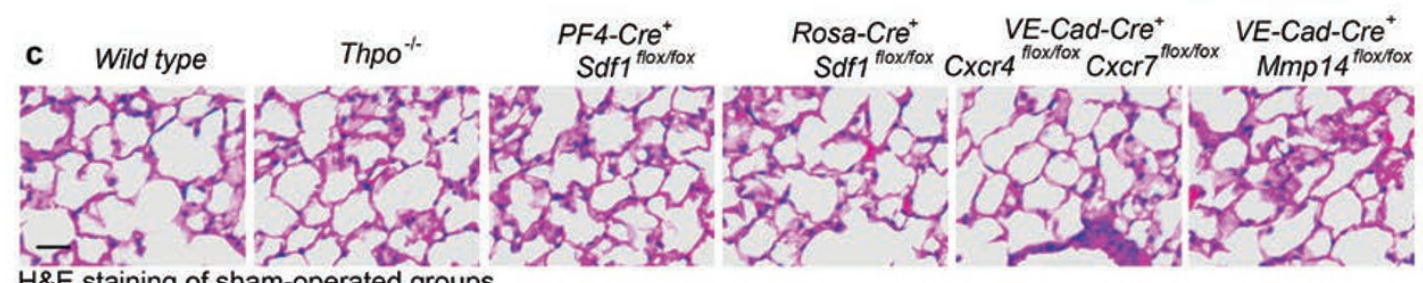
H\&E staining of sham-operated groups

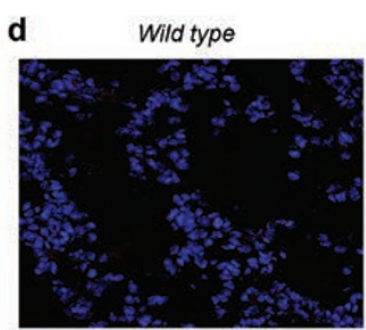

Rosa-Cre ${ }^{+}$ Sdf1 floxffox

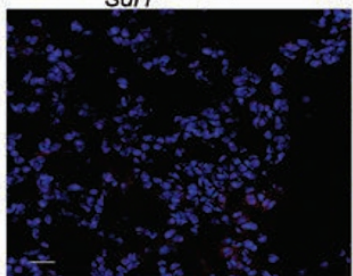

Sham groups (BrdU DAPI)

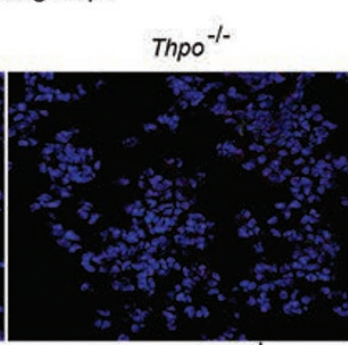

VE-Cad-Cre ${ }^{+}$ Cxcr4 flox/fox Cxcr 7 flox/fox

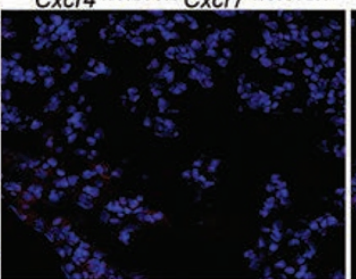

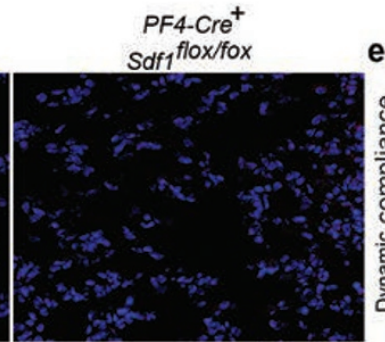

VE-Cad-Cre ${ }^{+}$ Mmp14 flox/fox

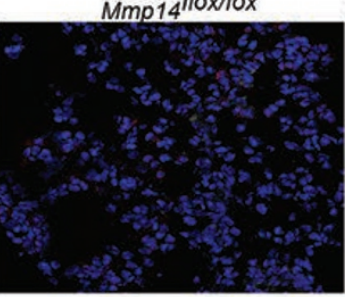

e

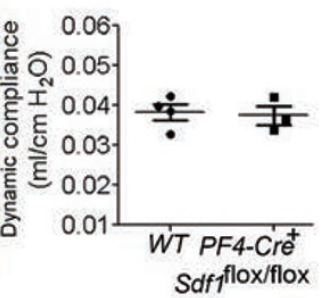

f

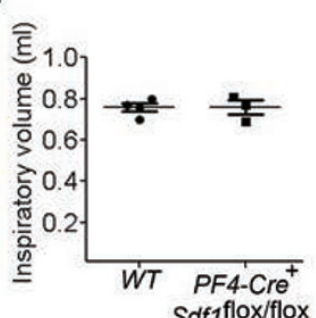

Supplementary Figure 1 Characterization of the lungs of indicated mouse genotypes after left lung pneumonectomy (PNX) and sham-operation. a, b) Protein levels of fibroblast cell markers desmin (a) and collagen (b) were tested by immunostaining and sirius red staining at indicated time points after PNX. Fibrotic mouse lungs at day 21 after intratracheal injection of Bleomycin (I.T. Bleo) was used as positive control. Scale bar $=50 \mu \mathrm{m}$. c, d) Sham operation was performed by thoracotomy without resection of left lung tissues. To introduce inducible or cell type-specific deletion of $S d f 1$, floxed $S d f 1$ mice were crossed with mice harboring platelet-specific PF4-Cre (PF4-Cre ${ }^{+}$Sdf $1^{\text {flox }}$ flox) and Rosa-Cre ERT2 (Rosa-Cre ${ }^{+} S d f 1^{\text {flox/flox }}$. Mice with endothelial cell (EC)specific VE-cadherin-Cre ${ }^{\text {ERT2 }}$ were also bred with mice carrying floxed SDF1 receptors Cxcr4 and Cxcr7 (VE-Cad-Cre+ $C x c r 4^{\text {flox/flox }} \mathrm{Cxcr}$ fflox/flox) and floxed Mmp14 mice ( $V E-C a d-C r e^{+} M m p 14^{\text {flox/flox }}$ ). Lung morphology was assessed by hematoxylin and eosin (H\&E) staining (c), and cellular proliferation was measured by staining incorporation of injected BrdU in the cryosection (d). Scale bar $=50 \mu \mathrm{m} . \mathrm{e}, \mathbf{f})$ Pulmonary function assay such as inspiratory volume (e) and compliance ( $f$ ) was performed to compare the respiratory capacity between wild type and PF4-Cre+Sdf1 flox/flox mice after sham operation. $\mathrm{n}=$ 4 mice in WT group and $=3$ mice for $P F 4-C r e^{+} S d f 1^{\text {flox/flox }}$ group. $P=0.38$ (e) and 0.98 ( $f$ ) between WT and mutant groups. Error bar denotes standard error of mean (s.e.m.), and line represents mean for panels e, f. All individual groups were compared by unpaired two tail t-test throughout this study. 
a

BrdU incorporation in the lungs (PNX Day 7)

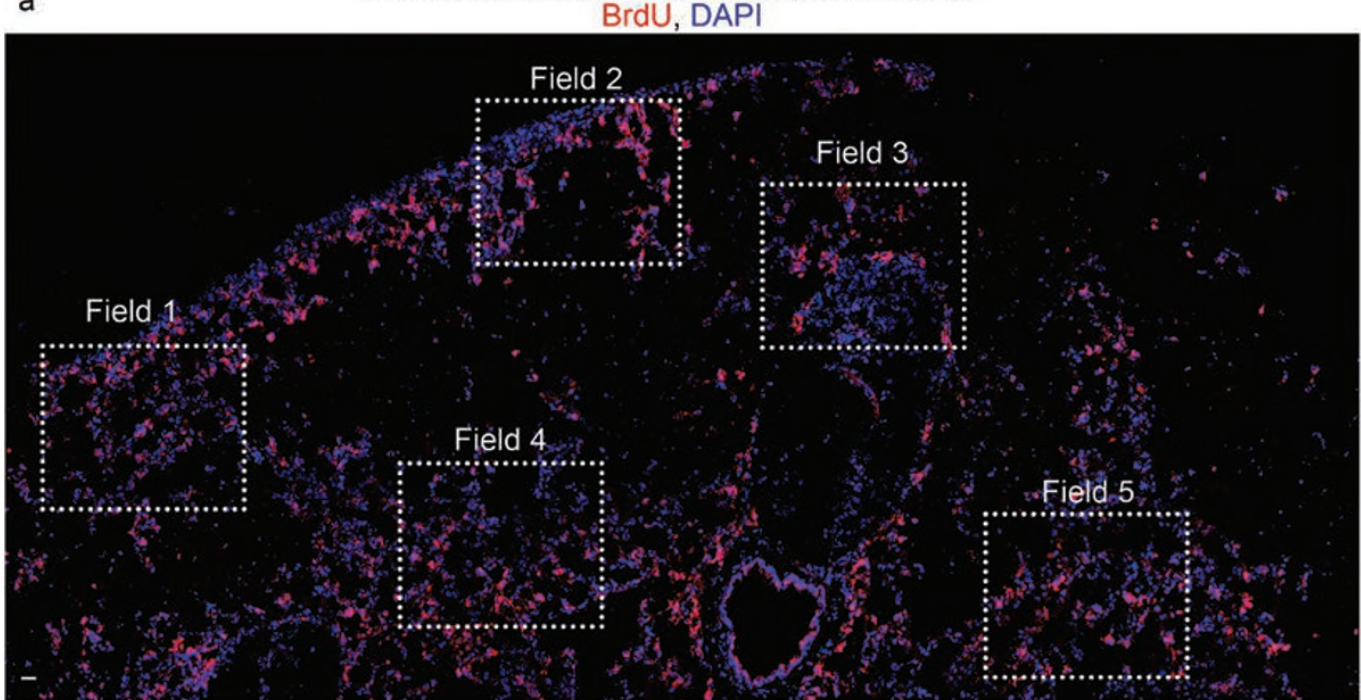

b

C
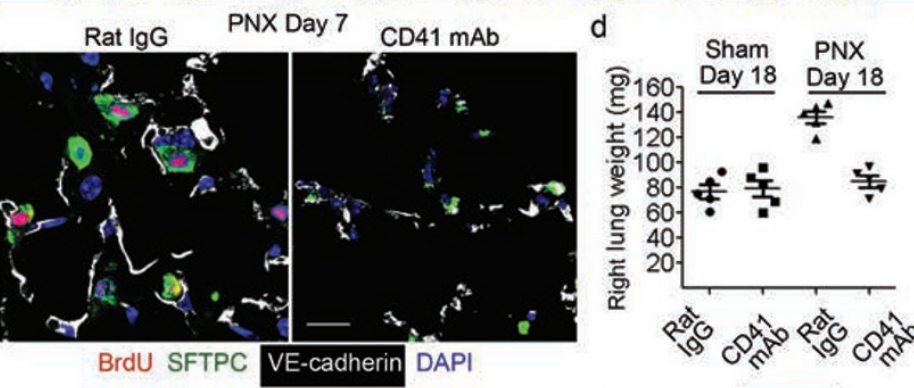

e

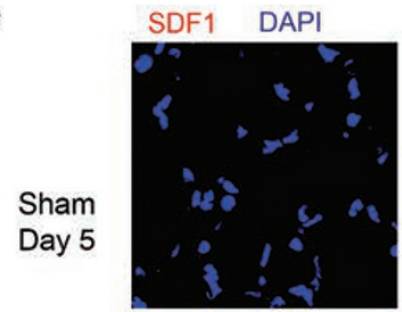

CD41 DAPI
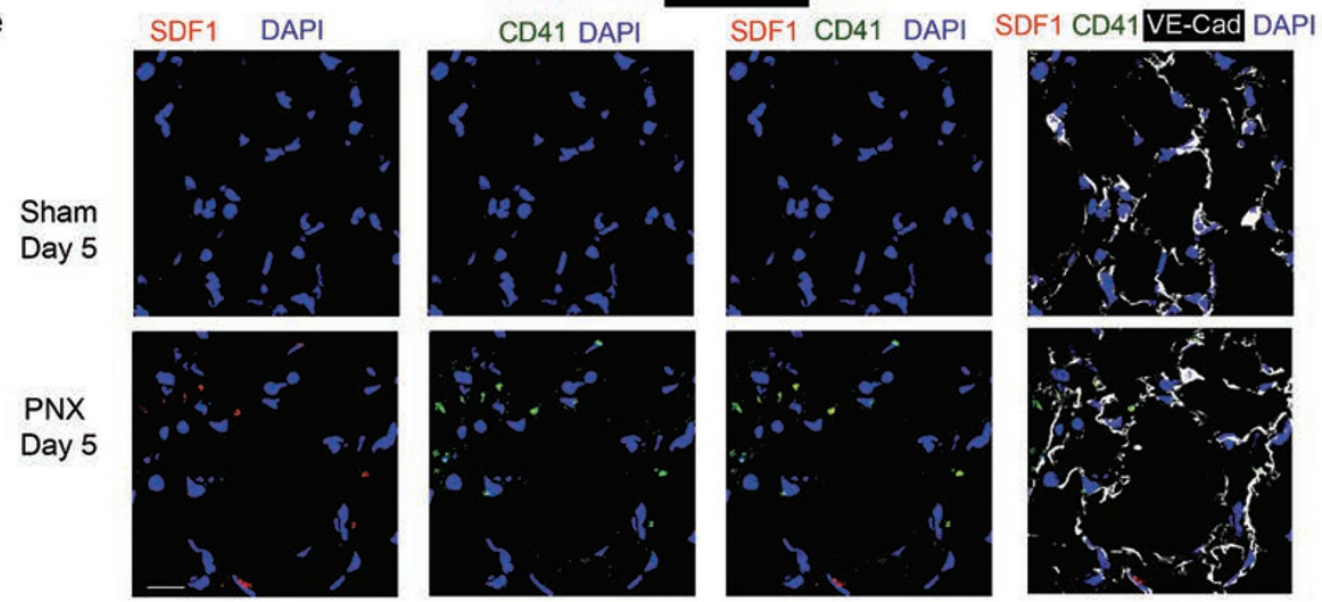

Supplementary Figure 2 Contribution of platelets in driving lung alveolar regeneration induced by PNX. a) Global view of BrdU immunostaining in the residual lung lobe at after PNX. Cellular proliferation was analyzed by staining of BrdU incorporation. Five fields were randomly chosen to quantify incorporation of $\mathrm{BrdU}$ as indicated; Scale bar $=50 \mathrm{um}$. b) Depletion of platelets in adult mice by injection of rat anti-mouse CD41 monoclonal antibody (mAb). Platelet counts in mice were determined at indicated time points after injection of CD41 mAb and same amounts of rat IgG control. Injection of CD41 $\mathrm{mAb}$ caused statistically lower number of circulating platelet count than IgG treated groups in all tested time points after injection; * $P<0.01$, compared with IgG-treated mice; $n=6$ mice per group. $c$, d) Proliferation of surfactant protein C (SFTPC) type 2 alveolar epithelial cells (AEC2s) (c) and regrowth of lung mass (d) in platelet-depleted and control mice after PNX. Representative immunostaining image is shown, and quantification of proliferating AEC2s and VE-cadherin ${ }^{+}$PCECs is presented in Fig. $1 . \mathrm{n}=5$ mice in all groups, and $P=$ 0.000665 between IgG and CD41 mAb (PNX group). Scale bar $=50 \mu \mathrm{m}$. e) PNX caused enrichment of SDF $1{ }^{+} \mathrm{CD} 41^{+}$platelets on VE-cadherin ${ }^{+}$pulmonary capillary endothelial cells (PCECs) on day 5. By contrast, sham-operated mice receiving only thoracotomy exhibited lower platelet deposition on PCECs; Scale bar $=50 \mu \mathrm{m}$. Error bar depicts s.e.m., and line stands for mean for panels b, d. Statistical difference between individual groups was determined by unpaired two tail t-test throughout this study. 
a

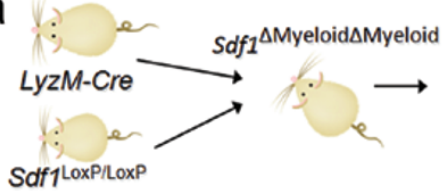

Sdf1LoxP/LoxP

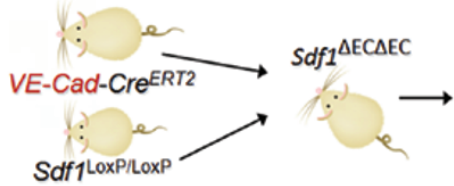

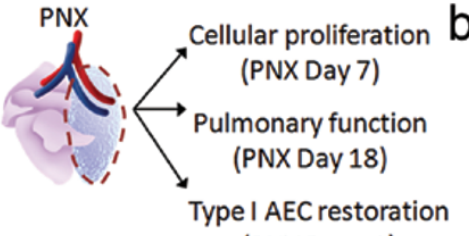

(PNX Day 18)

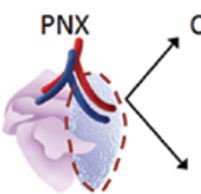

Cellular proliferation

(PNX Day 7)

Epithelialization

(PNX Day 18)
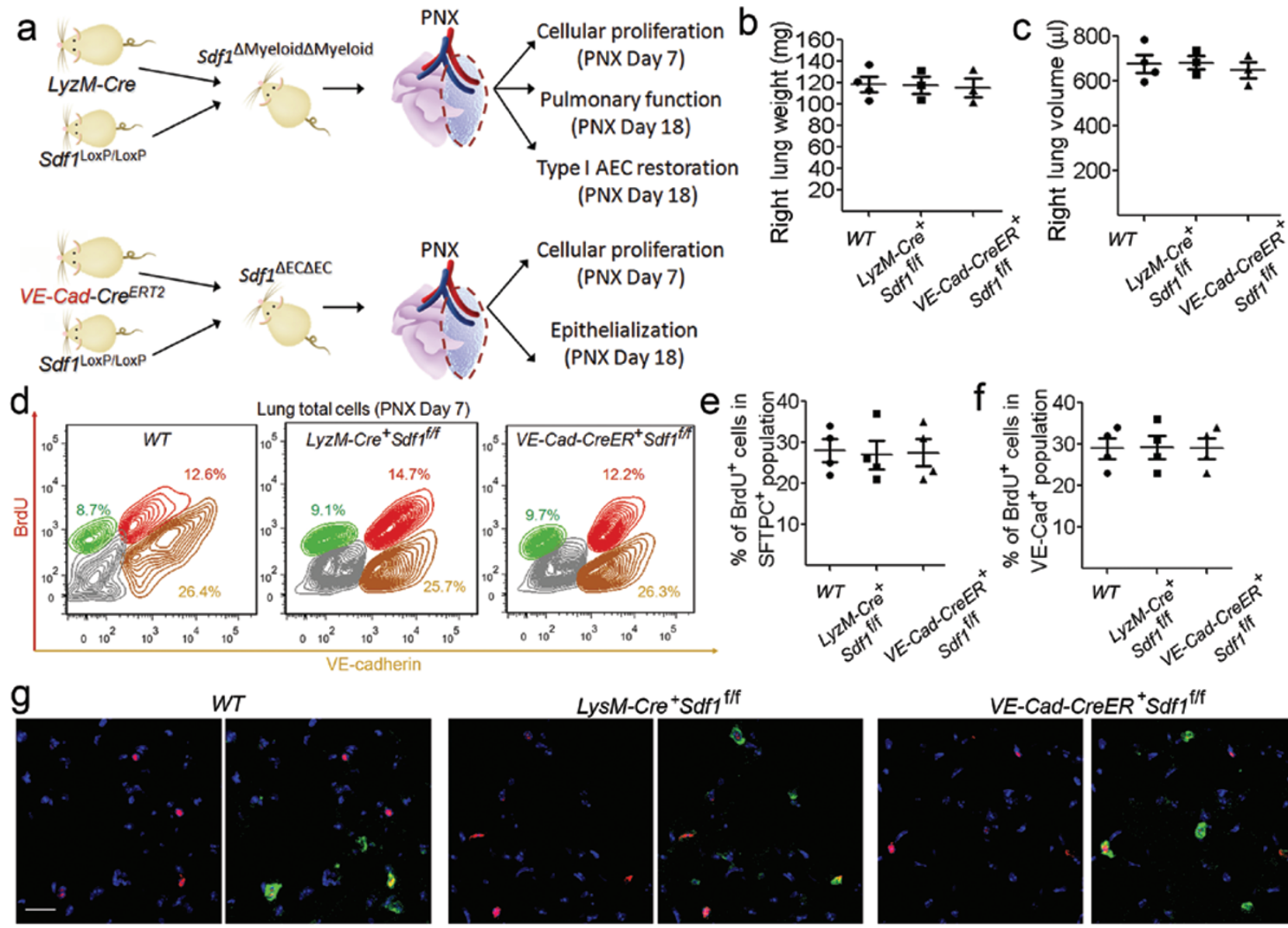

WT

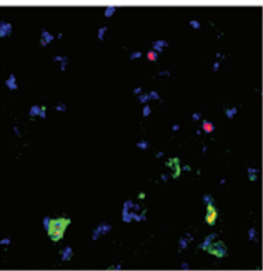

LysM-Cre ${ }^{+}$Sdf $1^{\mathrm{f} / \mathrm{f}}$
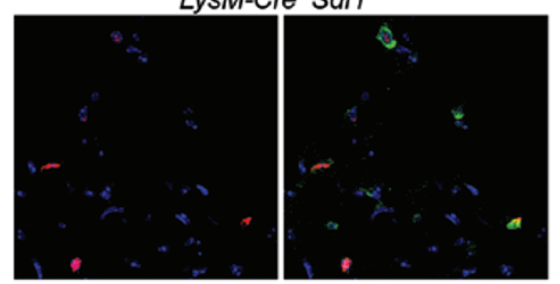

PNX Day 7 (BrdU SFTPC DAPI) h

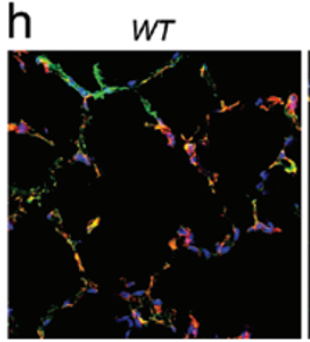

PNX Day 18 (Aquaporin-5 Podoplanin

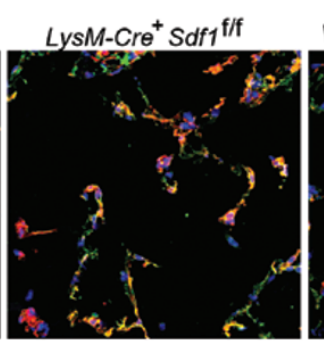

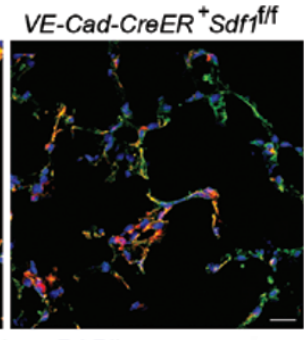

DAPI)

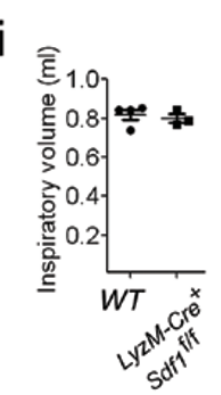

VE-Cad-CreER ${ }^{+} S d f 1^{f / f}$
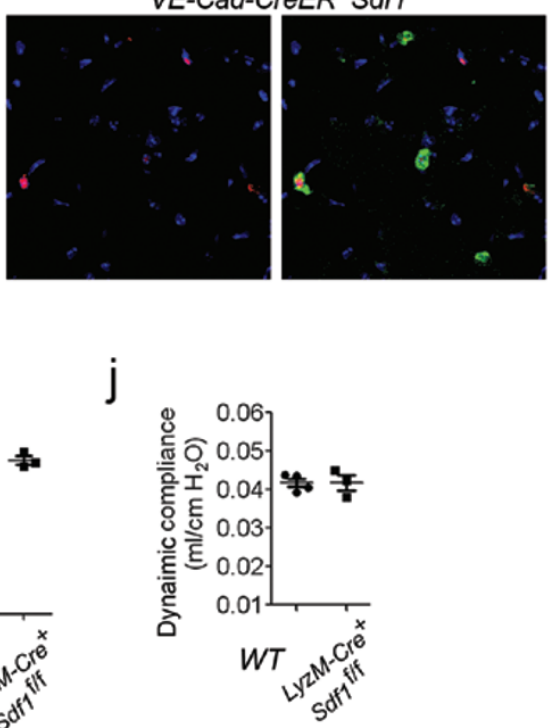

Supplementary Figure 3 Genetic approaches to illustrate cell type-specific contribution of SDF1 in promoting lung regeneration. a) Floxed Sdf1 mice were crossed with myeloid cell-specific LySM-Cre, VE-cadherin-Cre ETR2 mice to generate mice lacking Sdf1 in myeloid and endothelial cells (ECs). Resultant myeloid- and EC-specific Sdf1 knockout mice ( $L y s M-C r e^{+} S d f 1^{f / f}$ and VE$\mathrm{Cad}-\mathrm{Cr} e^{\mathrm{ER}+} \mathrm{Sdf} \mathrm{f}^{\mathrm{fff}}$ ) underwent PNX, and lung regenerative responses were tested and compared._b-j) Regeneration parameters were compared between wild type, myeloid- and EC-specific Sdf1 knockout mice ( $L y s M-C r e^{+} S d f 1^{f / f}$ and $V E-C a d-C r e^{E R+} S d f 1^{f / f}$ ) after PNX, including increase in right lung weight (b) and volume (c), proliferation/BrdU incorporation of PCECs and AEC2s (d-g), recovery of type 1 alveolar epithelial cell (AEC1) (h), and restoration of pulmonary function ( $\mathrm{i}, \mathrm{j})$. In (b) and (c), $\mathrm{n}=4$ mice in WT group and 3 animals for both $\mathrm{LySM}^{-\mathrm{Cre}}{ }^{+} \mathrm{Sdf} \mathrm{1}^{\mathrm{f} / \mathrm{f}}$ and $\mathrm{VE}-\mathrm{Cad}-\mathrm{Cr} \mathrm{E}^{\mathrm{ER}+} \mathrm{Sdf} \mathrm{f}^{\mathrm{flf}}$ groups. In (e) and (f), $\mathrm{n}=4$ animals in all tested genotypes. In (i) and (j), $n=4$ mice in WT and 3 mice in $\mathrm{LysM}-\mathrm{Cre}^{+} \mathrm{Sdf} \mathrm{1}^{\mathrm{fff}}$ groups. $P=0.48$ (i) and 0.86 (j) between these two groups; Scale bar $=50 \mu \mathrm{m}$. Error bar defines s.e.m., and line represents mean for panels b, c, e, f, i, j. Statistical difference between individual groups was assessed by unpaired two tail t-test throughout this study. 
a
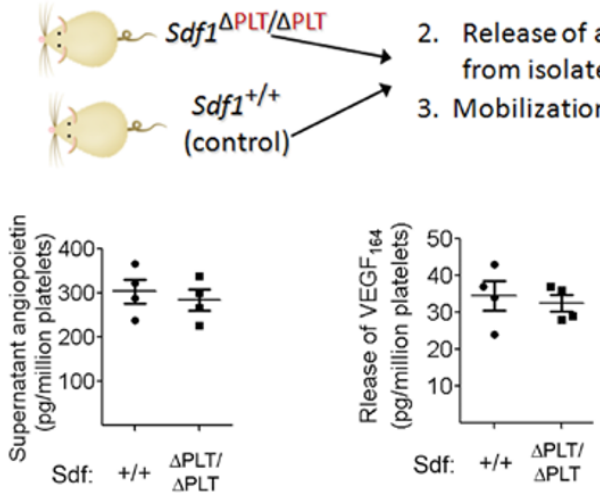

1. Accumulation of $\mathrm{Gr} 1^{+} \mathrm{CD} 45^{+}$myeloid cells

2. Release of angiogenic factors from isolated platelets

3. Mobilization of P-selectin in isolated platelets

Basic FGF released by activated platelets

C
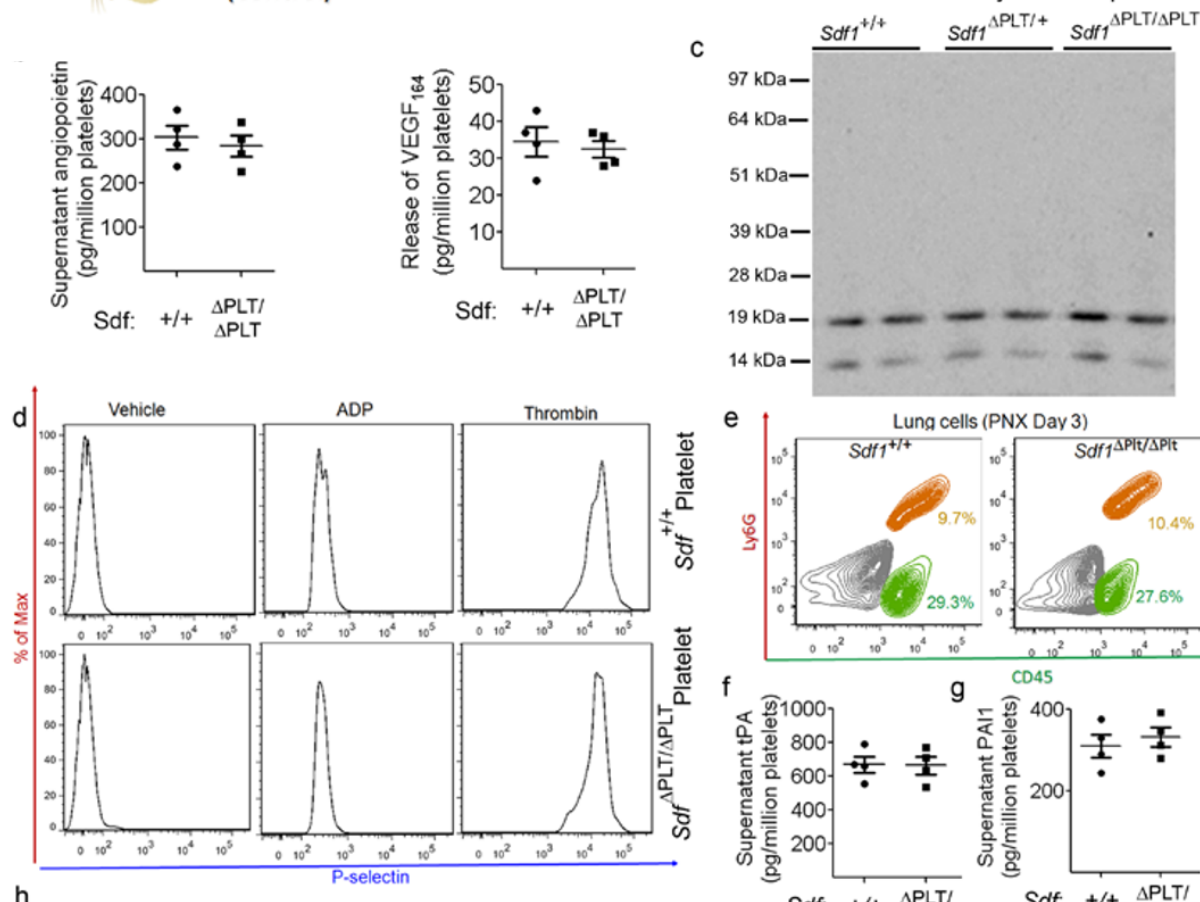

$\mathrm{h}$

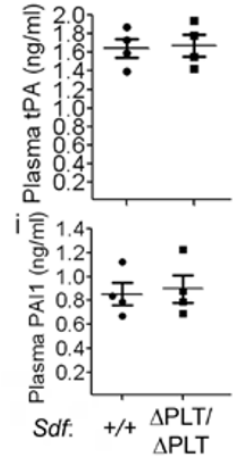

e

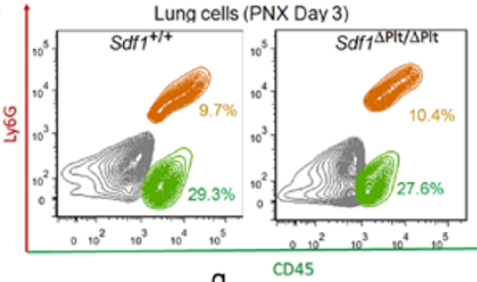

$\mathrm{g}$
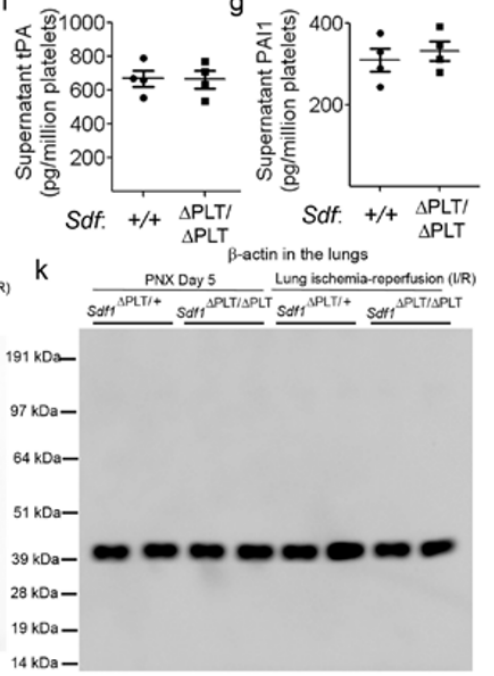

$\beta$-actin in the lungs
Supplementary Figure 4 Strategy to compare paracrine factor deployment and coagulation in wild type $\left(S d f 1^{+/+}\right)$and Sdf1-deficient $\left(S d f 1^{-/}\right)$platelets. a) Mouse line with platelet/platelet progenitor-specific platelet factor 4 promoter-driven Cre (PF4-Cre) was crossed with $S d f 1^{\text {loxP/loxP }}$ mice to delete $S d f 1$ in platelets ( $\left.S d f 1^{\Delta \mathrm{PLT} / \Delta \mathrm{PLT}}\right)$. Control and Sdf1-deficient ( $S d f 1^{-/-}$) platelets were isolated from wild type and $S d f 1^{\triangle P L T / \triangle P L T}$ mice and treated with agonists. Subsequent deployment of angiogenic factors, mobilization of $\mathrm{P}$-selectin was determined in isolated platelets, and accumulation of $\mathrm{Gr} 1^{+} \mathrm{CD} 45^{+}$myeloid cells was measured in control and $S d f 1^{\triangle \mathrm{PLT} / \Delta \mathrm{PLT}}$ mice after PNX._b, c) Protein levels of VEGF 164 (a), angiopoietin2 (b), and FGF-2 (c) in supernatant of WT and $S d f 1^{-/-}$platelets after ADP stimulation. Protein amount was determined by immunoblot (R\&D and Abcam). Platelets were stimulated with $10 \mu \mathrm{mol} / \mathrm{L}$ ADP. (b) $\mathrm{n}=4$ mice in both groups, $P=0.74$ between VEGF levels of both groups and $=0.67$ between angiopoietin2 levels of two genotypes. d) Surface mobilization of P-selectin in wild type and $\mathrm{Sdfl}^{-/}$platelets was determined by flow cytometry after stimulation of $\mathrm{ADP}$ and $0.1 \mathrm{U} / \mathrm{mL}$ thrombin._e) Pulmonary accumulation of $\mathrm{Gr} 1^{+} \mathrm{CD} 45^{+}$ myeloid cells in control and Sdf1 1 PLT/ $\triangle \mathrm{PLT}$ mice after PNX was measured by flow cytometry and the percentage of myeloid cells in the lungs was compared. $\mathbf{f}, \mathbf{g}$ ) Protein level of tissue-type plasminogen activator (tPA) and plasminogen activator inhibitor 1 (PAI1) in supernatant of $S d f 1^{+/+}$and Sdf1deficient $\left(\mathrm{Sdfl}^{-{ }^{-}}\right.$) platelets. Platelet activation and protein detection was performed as panels $c$ (Abcam and R\&D). $n=4$ animals in all groups shown in ( $f$ and $g$ ), $P=0.95$ ( $\mathrm{f}$ ) and 0.47 (g) between two genotypes. $\mathrm{h}$, i) Plasma level of tPA and PAI1 was also measured in control and Sdf1 $\triangle \mathrm{PLT} / \triangle \mathrm{PLT}$ mice. $\mathrm{n}=4$ mice in all groups presented, $P=0.34(\mathrm{~h})$ and 0.83 (i) between two genotypes. j, k) Formation of fibrin clots in the lungs was determined after both PNX and lung injury caused by ischemia-reperfusion (I/R). Fibrin deposition in individual mice after $150 \mathrm{~min}$ I/R procedure was determined by immunoblot analysis of fibrin $\beta$-chain extracted from lung lysates (antibody 350 from American Diagnostica). Mouse left lung was subjected to 30 minute ischemia by clamping the left lung hilum and then reperfused for 120 minutes after removing vascular clamp. Protein level of $\beta$-actin in the lung lysates after I/R was measured to control for equal amounts of sampling loading. Procedure of mouse lung I/R and extraction of fibrin clot in the lungs was described in "Methods". In the shown immunoblot image, each lane describes individual mouse. Utilized mice were from two cohorts. Error bar indicates s.e.m., and line stands for mean for panels $f$, $\mathrm{g}, \mathrm{h}, \mathrm{i}$. Unpaired two tail t-test was used to determine statistical difference between groups. 


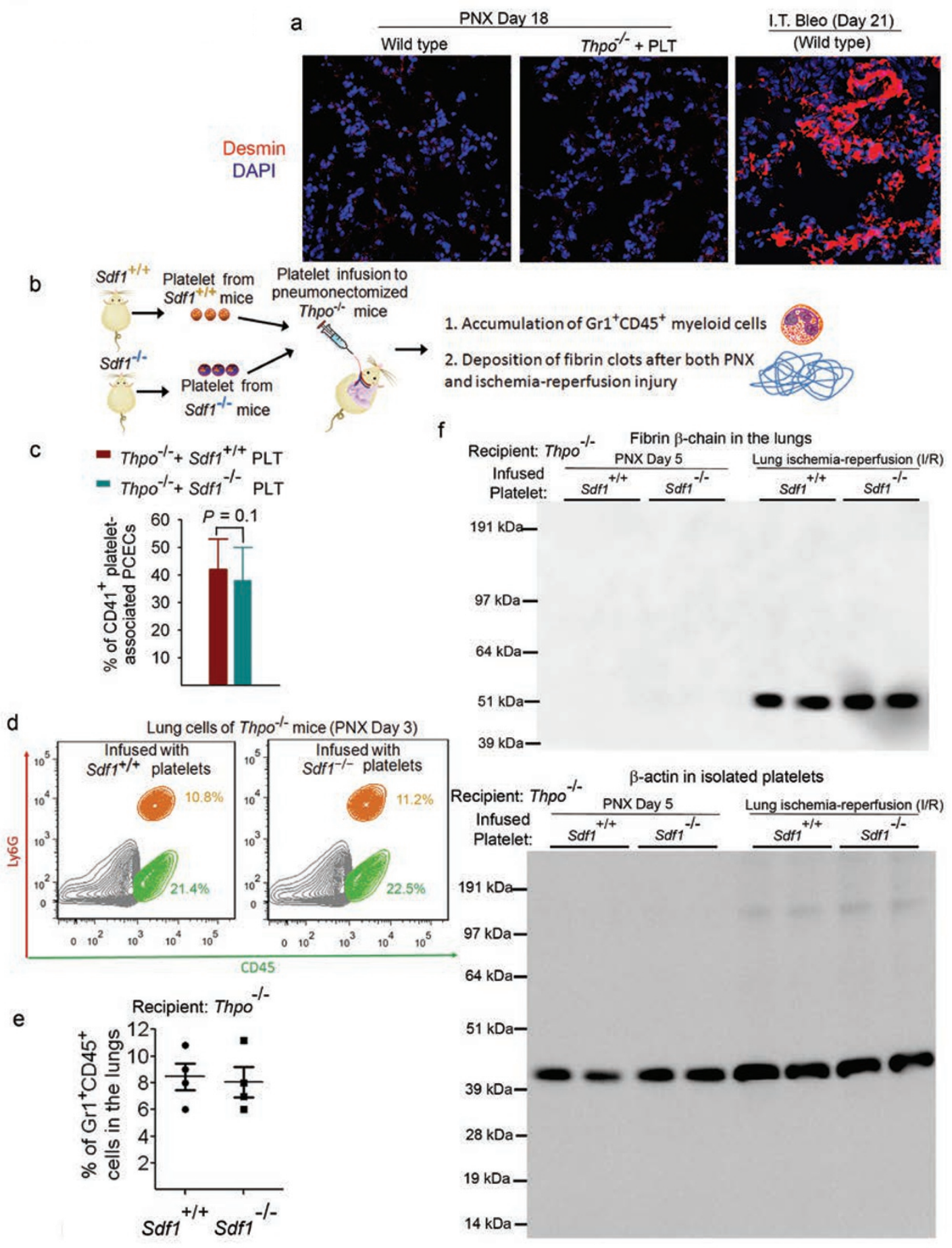

Supplementary Figure 5 Inflammatory and thrombotic responses in Thpomice transplanted with wild type and Sdf1-deficient platelets. a) Activation of fibroblast cells in Thpo-/ mice after PNX and transplanted with platelets. Protein level of fibroblast cell marker desmin was tested by immunostaining in recipient $\mathrm{Thpo}^{-/}$mice after PNX and platelet transplantation. Mouse lungs after intratracheal injection of Bleomycin (I.T. Bleo) at day 21 was also employed as positive control. Scale bar $=50 \mu \mathrm{m}$. b) Approaches to compare inflammatory and thrombotic responses in the lungs of pneumonectomized $\mathrm{Thpo}^{-/-}$mouse after intravascular transfusion of $\mathrm{Sdf1}^{+/+}$and $\mathrm{Sdf1}^{-l_{-}}$platelets. $S d f 1^{+/+}$and $S d f 1^{-/-}$platelets were infused into $T h p o^{-/-}$mice via jugular vein, respectively. c) Comparable extent of pulmonary recruitment and distribution of infused $\mathrm{Sdf1}^{+/+}$and $\mathrm{Sdf1^{-/ }}$ platelets. Recruitment of $\mathrm{CD} 41^{+} \mathrm{Sdf1^{+/+ }}$ and
$S d f 1^{-/-}$platelets in the lung capillary was assessed by quantifying association with VE-cadherin ${ }^{+}$PCECs; $n=5$ mice._d, e) Pulmonary accumulation of $\mathrm{Gr} \mathrm{I}^{+} \mathrm{CD} 45^{+}$myeloid cells in Thpo-/- mice transplanted with $\mathrm{Sdf1} \mathrm{I}^{+/+}$and $S d f 1^{-/-}$platelets. Flow cytometry was used to determine the percentage of $\mathrm{Gr} 1^{+} \mathrm{CD} 45^{+}$myeloid cells in the lungs after PNX. $\mathrm{n}=4$ mice in both groups and $P=0.83$ between two groups._f) Fibrin deposition in the lungs of Thpo/- mice transplanted with $S d f 1^{+/+}$and $S d f 1^{-/-}$platelets. Recipient mice underwent PNX or acute lung I/R injury (150 min). Deposition of fibrin clots was assessed by immunoblot of fibrin $\beta$-chain. In the presented immunoblot image, each lane indicates sample from individual mouse. Error bar stands denotes s.e.m., and line represents mean for panel e. Statistical difference between groups was assessed by unpaired two tail t-test. 

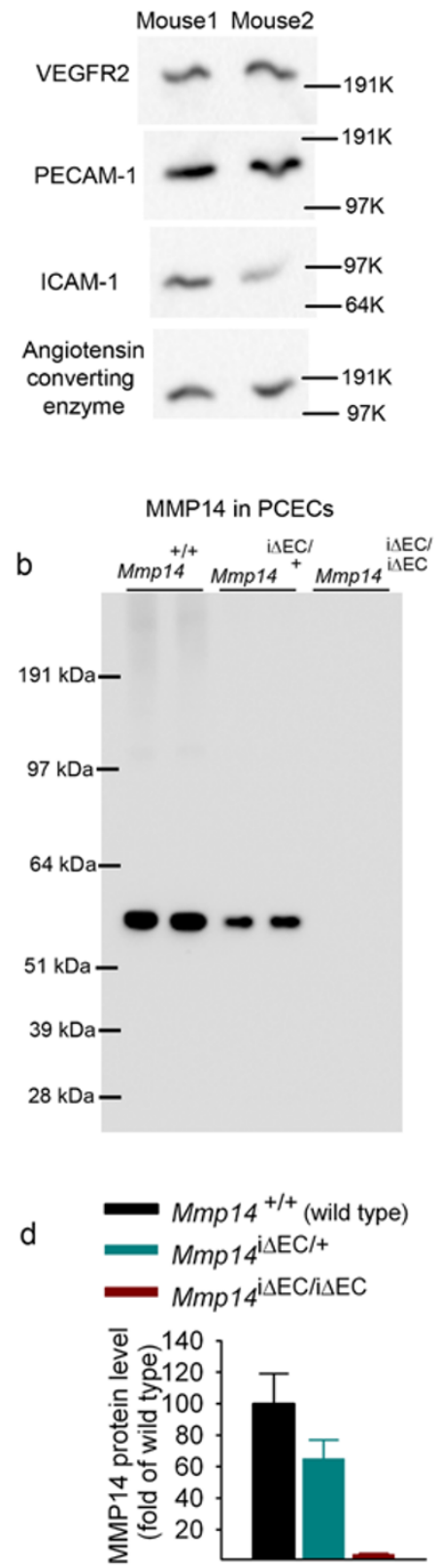
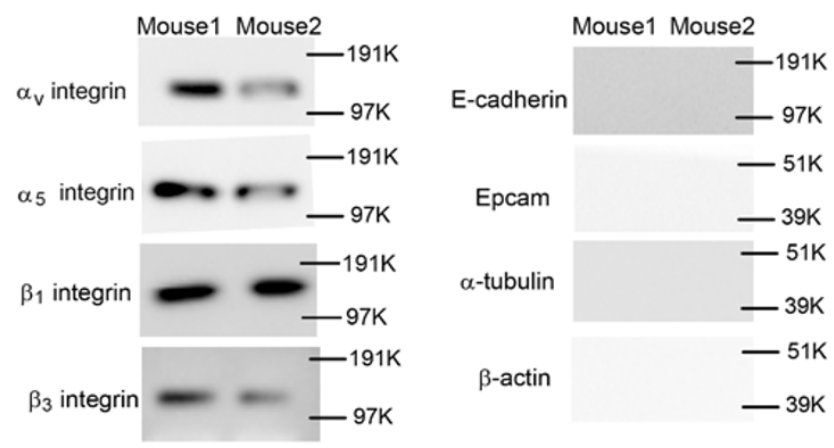
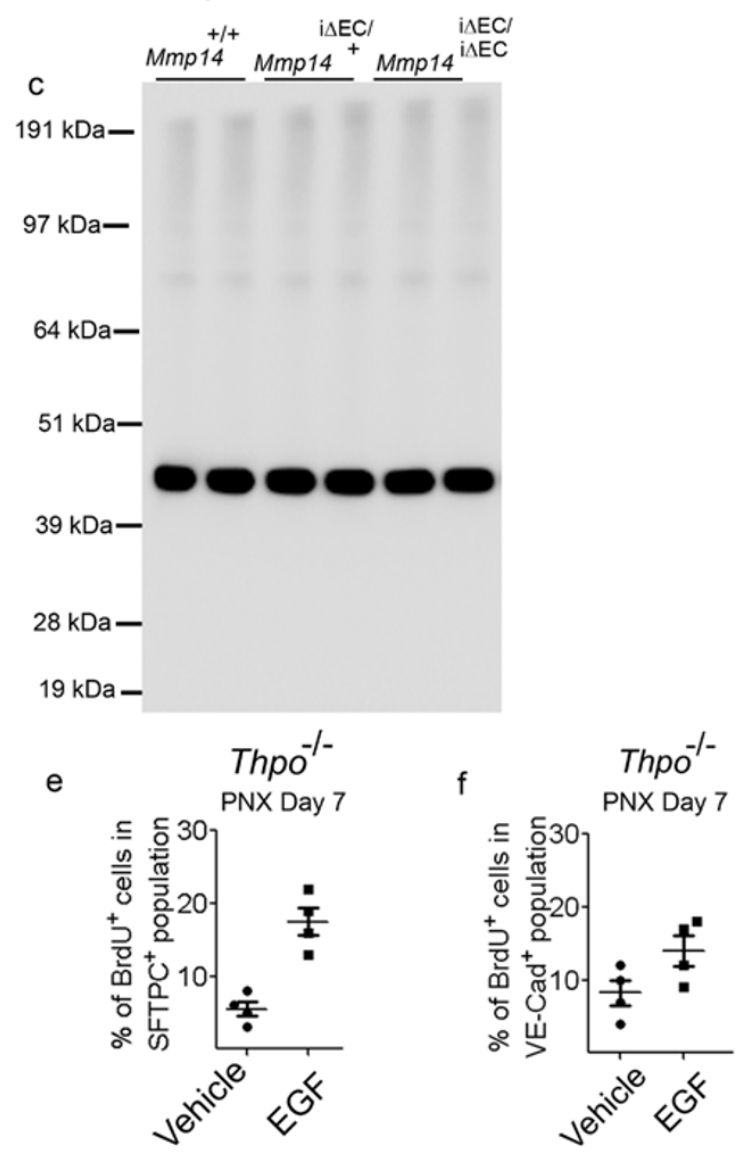

\begin{abstract}
Supplementary figure 6 Characterization of isolated surface proteins of mouse PCECs and inducible deletion of Mmp14 specifically in ECs of adult mice. a) Mouse PCEC membrane proteins were biotinylated by performing pulmonary perfusion with EZ-Link Sulfo-NHS-LC-Biotin (Pierce) that labels protein free amine groups. Biotinylated proteins were isolated by streptavidin conjugated beads. Immunoblot was used to analyze isolated endothelial surface antigens of PCECs, including vascular endothelial cell receptor 2 (VEGFR2), platelet endothelial cell adhesion molecule-1 (PECAM-1), intercellular adhesion molecule-1 (ICAM-1), angiotensin converting enzyme (ACE), integrin $\alpha v, \alpha 5, \beta 1, \beta 3$, E-cadherin and epithelial cell adhesion molecule (EPCAM). Cytoplasmic proteins such as $\beta$-actin and $\alpha$-tubulin were also tested by immunoblot to exclude the contamination of non-membrane
\end{abstract}

proteins in isolated PCEC membrane fractions. b-d) Endothelial cell (EC)specific inducible deletion of alveologenic paracrine factor Mmp14 in adult mice. VE-cadherin-Cre ${ }^{\mathrm{ETR} 2} / C d h 5-P A C$-Cre ${ }^{\mathrm{ETR} 2}$ mice were crossed with mice carrying floxed Mmp14. Deletion efficiency of target genes was measured by immunoblot in isolated PCECs and quantified after normalization to protein level of $\beta$-actin. $n=6$ mice per group. e-f) Proliferation of AEC2s and PCECs was tested in Thpo $^{-/}$mice after injection of MMP14 downstream effector EGF. $\mathrm{n}=4$ animals in all tested groups, and $P=0.002$ (e) and $0.012(\mathrm{f})$. In the presented immunoblot image, each lane indicates individual mouse. All mice were from two different cohorts. Error bar stands for s.e.m., and line represents mean for panels $d, e, f$. Statistical difference between groups was determined by unpaired two tail t-test. 
a

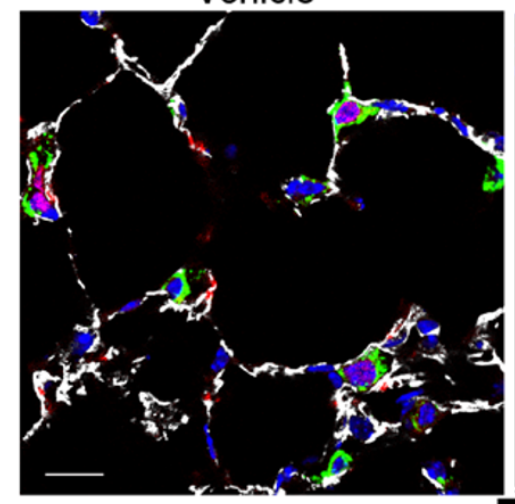

Wild type (PNX Day 7)

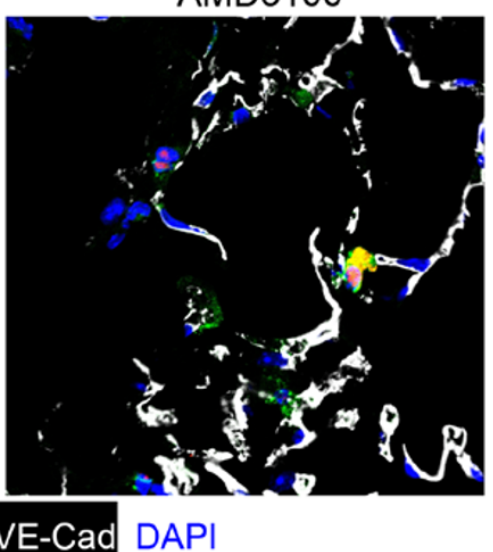

b

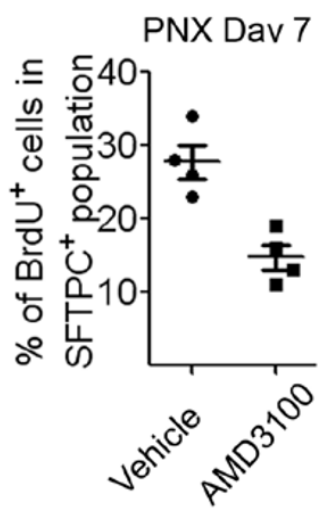

C

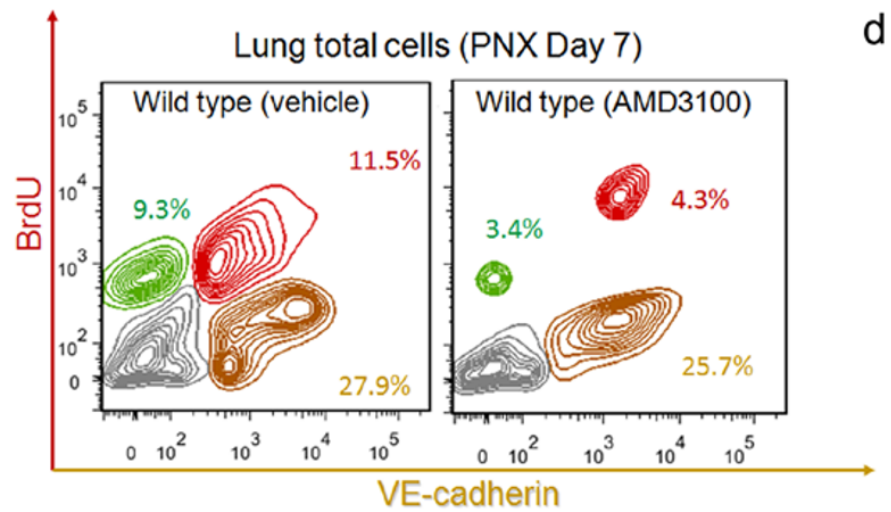

d

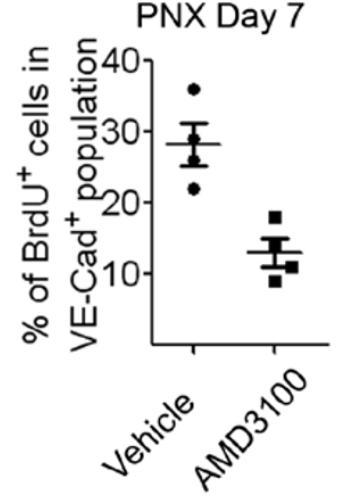

e PNX Day 18

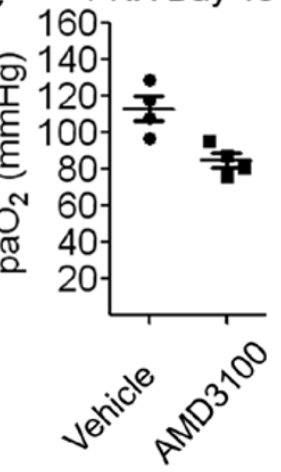

Supplementary Figure 7 Effect of CXCR4 antagonist AMD3100 on lung regeneration. a-d) Proliferation of SFTPC + AEC2s and VE-cadherin ${ }^{+}$PCECs in AMD3100-treated mice after PNX was determined. CXCR4 antagonist AMD3100 was subcutaneously injected into wild type mice at dose of 2 $\mathrm{mg} / \mathrm{kg}$ every two days, and proliferation was assessed by staining for BrdU incorporation, and proliferation of AEC2s and PCECs was determined by co- staining with SFTPC (a) and VE-cadherin flow cytometry (c). $n=4$ animals in all presented groups, and $P=0.0042$ (b) and 0.0051 (d). Scale bar $=$ $50 \mu \mathrm{m}$. e) Restoration of gas exchange function in mice after PNX after treatment of AMD3100. $\mathrm{n}=4$ animals in both groups, $P=0.043$. Error bar describes for s.e.m., and line represents mean for panels b, d, e. Unpaired two tail t-test was used to determine statistical difference between groups. 
a
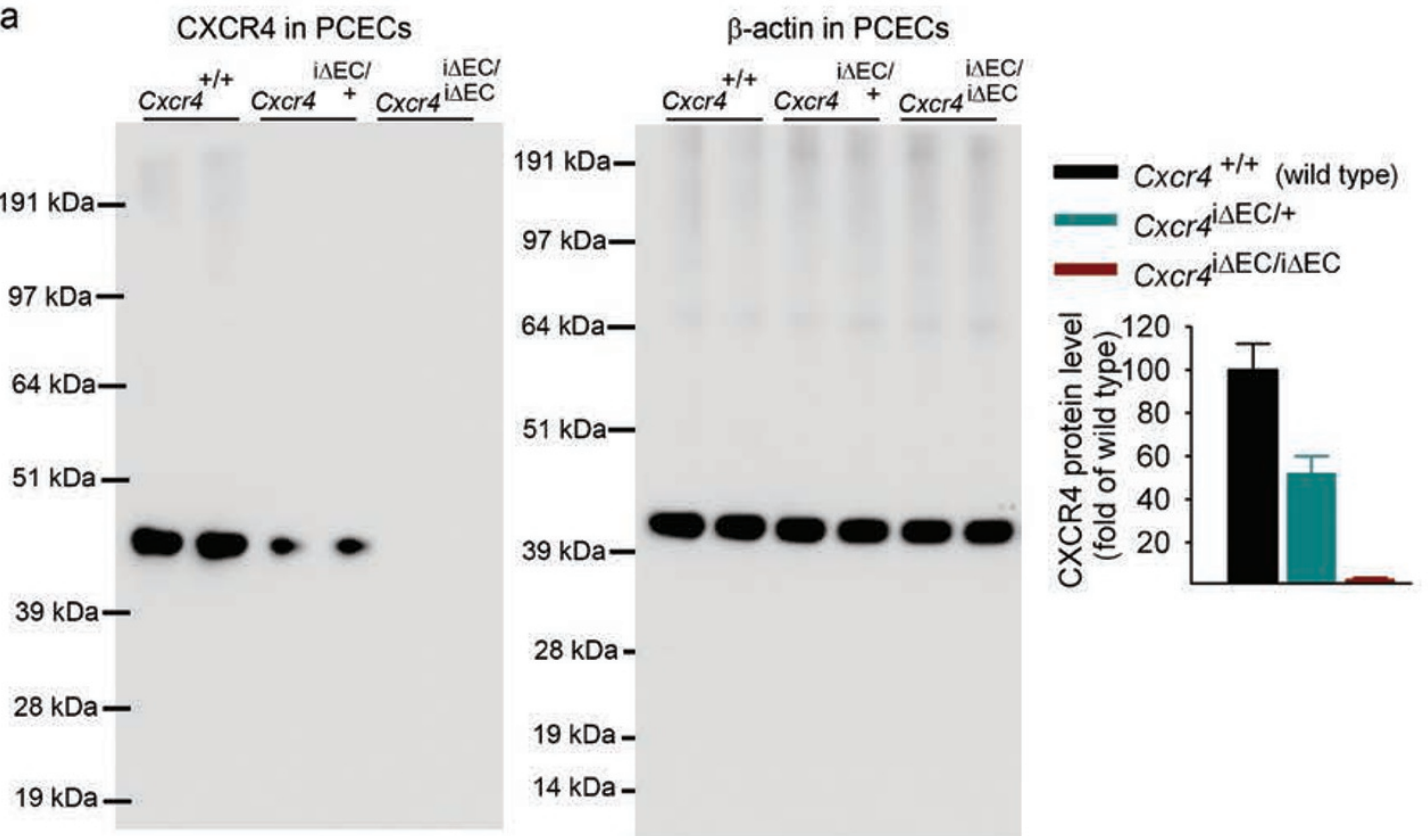

b

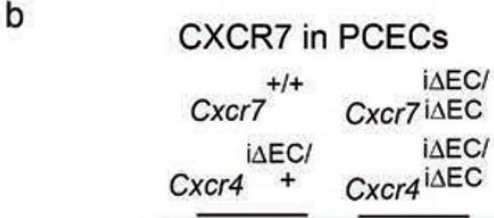

$28 \mathrm{kDa}-$

$19 \mathrm{kDa}-$

$14 \mathrm{kDa}-$

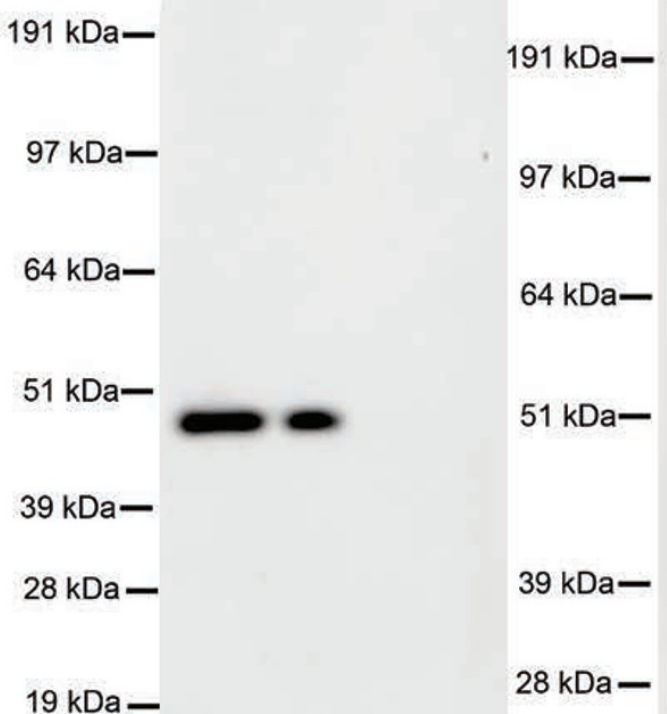

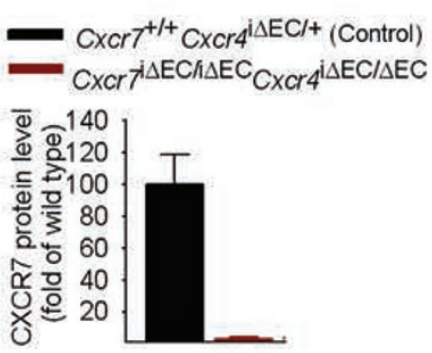

C

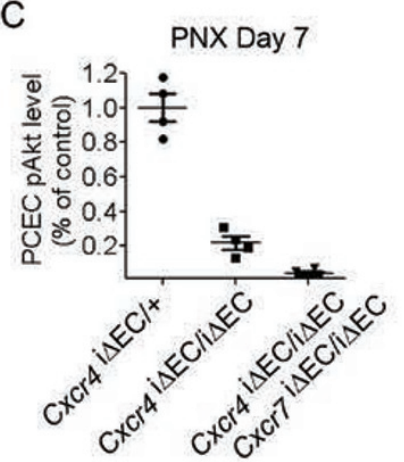

Supplementary Figure 8 Inducible endothelial cell (EC)-specific deletion of SDF1 receptors $\mathrm{CxCr} 4$ and $\mathrm{CxCr} 7$ in adult mice. a-b) VE-cadherin$\mathrm{Cre}^{\mathrm{ETR} 2} / \mathrm{Cdh}$ 5-PAC-Cre ${ }^{\mathrm{ETR} 2}$ mice were crossed with mice carrying floxed Cxcr4 and $\mathrm{Cxcr7}$. Tamoxifen treatment in the resultant mice led to EC-specific deletion of Cxcr4 (a) and $\mathrm{Cxcr} \mathrm{(b)}$ in adult mice. Deletion efficiency of target genes was measured by immunoblot in isolated PCECs after normalization to $\beta$-actin. Representative blot image is shown with corresponding protein markers. $n=6$ per mouse group, and CXCR4 and
CXCR7 protein level was reduced by $92 \%$ and $94 \%$, compared to $\mathrm{CXCr} 4^{+/+}$ and $\mathrm{CxCr} 7^{+/+}$littermate. c) Activation of Akt in PCECs of indicated mouse groups at day 7 after PNX. $\mathrm{n}=4$ mice in all groups, and $P=0.00012$ (control versus $C x C r 4^{\mathrm{i}} \triangle \mathrm{EC} / \mathrm{i} \Delta \mathrm{EC}$ ), $P=0.00002$ (control versus $C x C r 4^{\mathrm{i}} \Delta \mathrm{EC} /$ $\left.{ }_{i \triangle E C} C_{X C r}>\mathrm{i} \triangle \mathrm{EC} / \mathrm{A} \triangle \mathrm{EC}\right)$. In the presented immunoblot image, each lane indicates individual mouse. Error bar represents s.e.m., and line stands for mean in all panels. Statistical difference between groups was assessed by unpaired two tail t-test. 


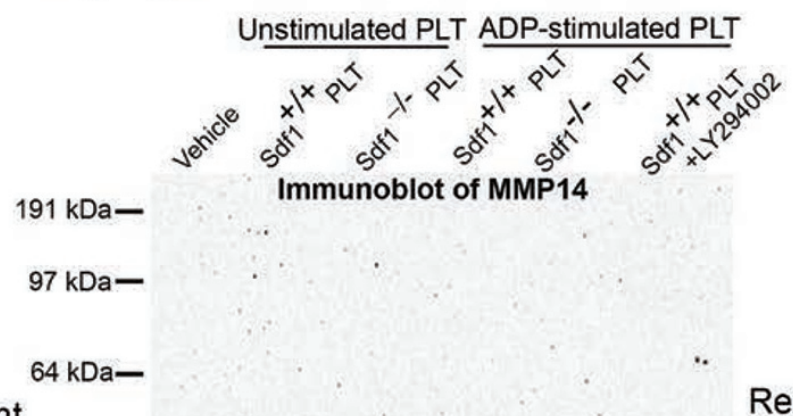

Relevant

to

Fig. $5 b$

$51 \mathrm{kDa}-$

$39 \mathrm{kDa}-$

$28 \mathrm{kDa}-$

$19 \mathrm{kDa}$ -

$191 \mathrm{kDa}-$

$97 \mathrm{kDa}-$

$64 \mathrm{kDa}-$

$51 \mathrm{kDa}-$

$39 \mathrm{kDa}-$

$28 \mathrm{kDa}-$

$19 \mathrm{kDa}-$

c

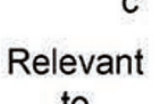

to

Fig. $5 e$

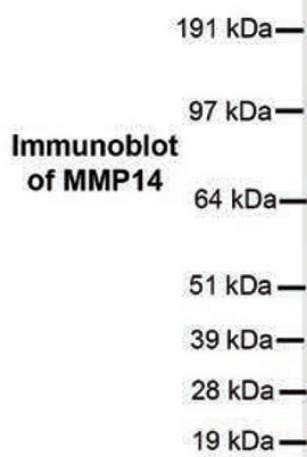

Human PCEC

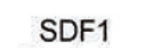
stimulation

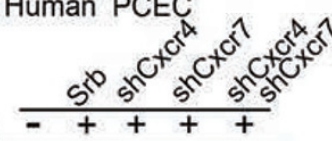

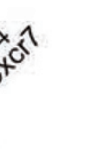

b

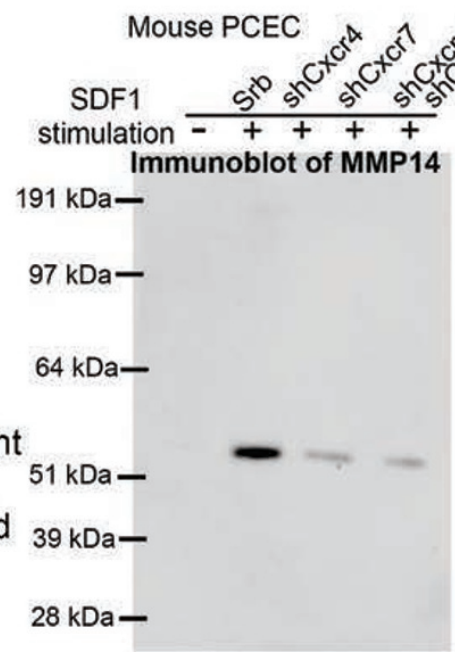

Immunoblot of $\beta$-actin

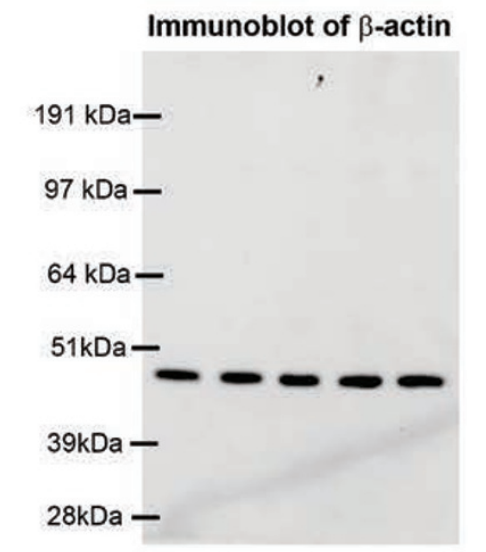

Human PCEC

Fig. 5 
d

Relevant to Fig. $5 g$

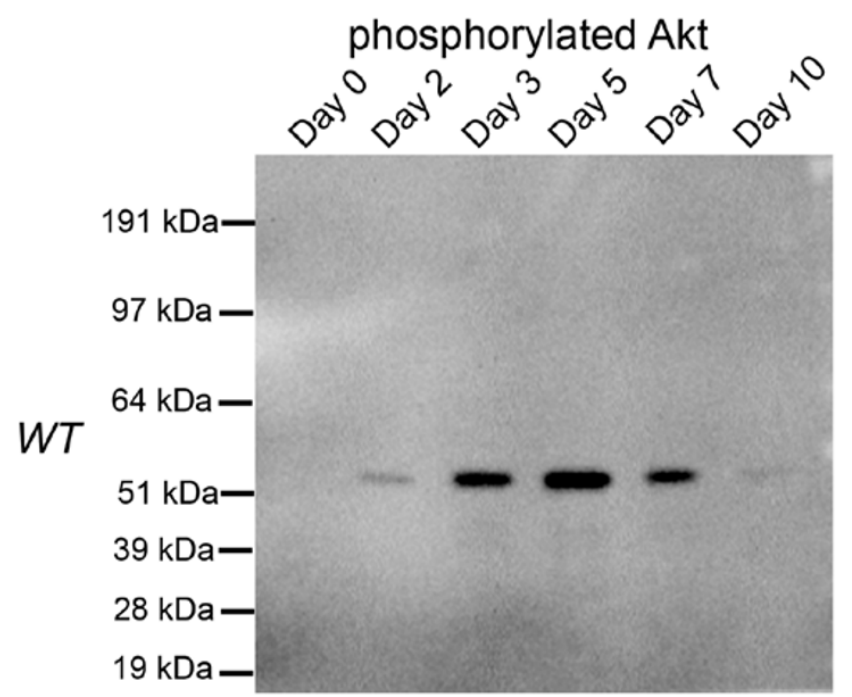

$$
\begin{aligned}
& 191 \mathrm{kDa}- \\
& 97 \mathrm{kDa}- \\
& 64 \mathrm{kDa}- \\
& 51 \mathrm{kDa}- \\
& 39 \mathrm{kDa}- \\
& 28 \mathrm{kDa}-
\end{aligned}
$$
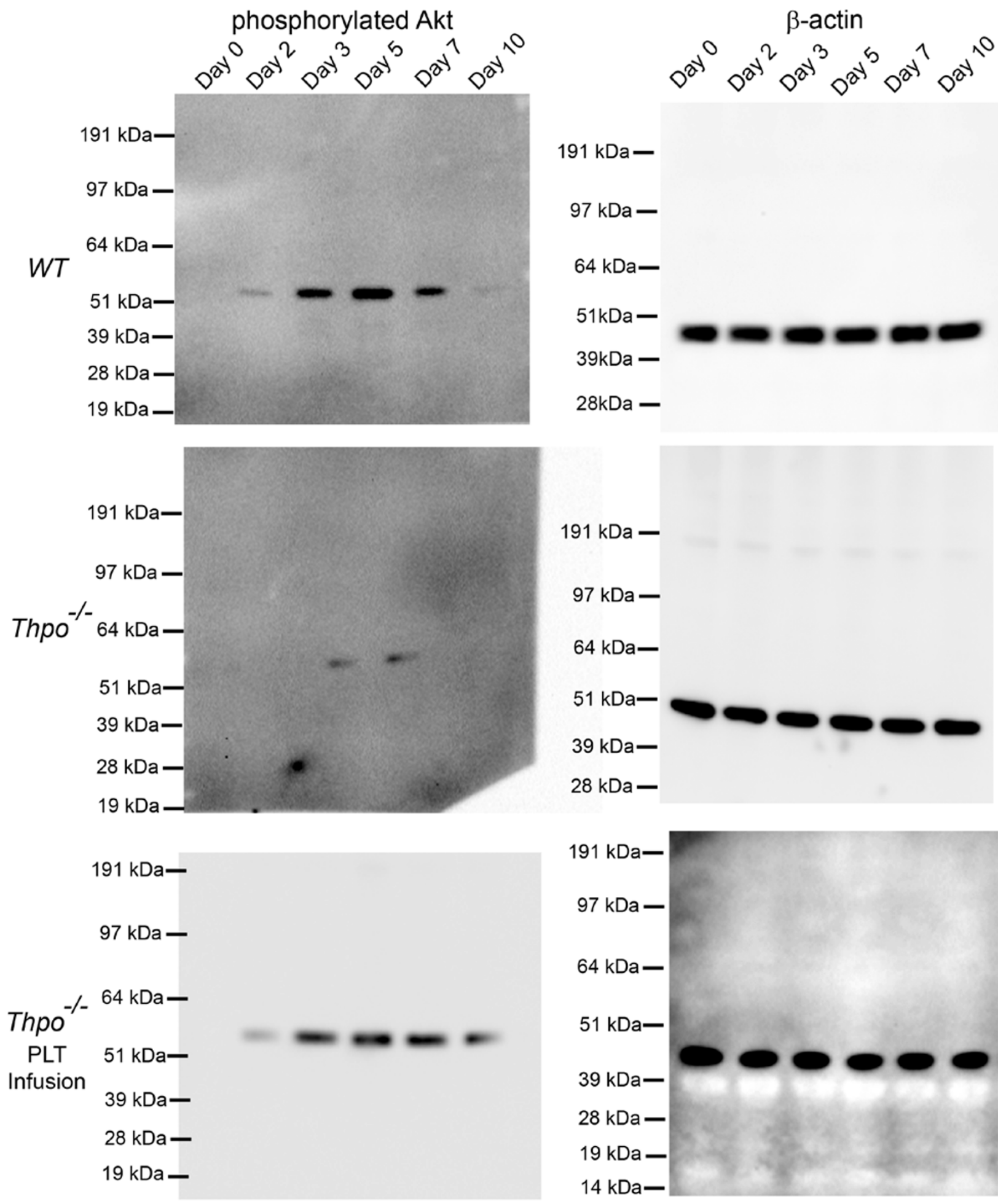
e

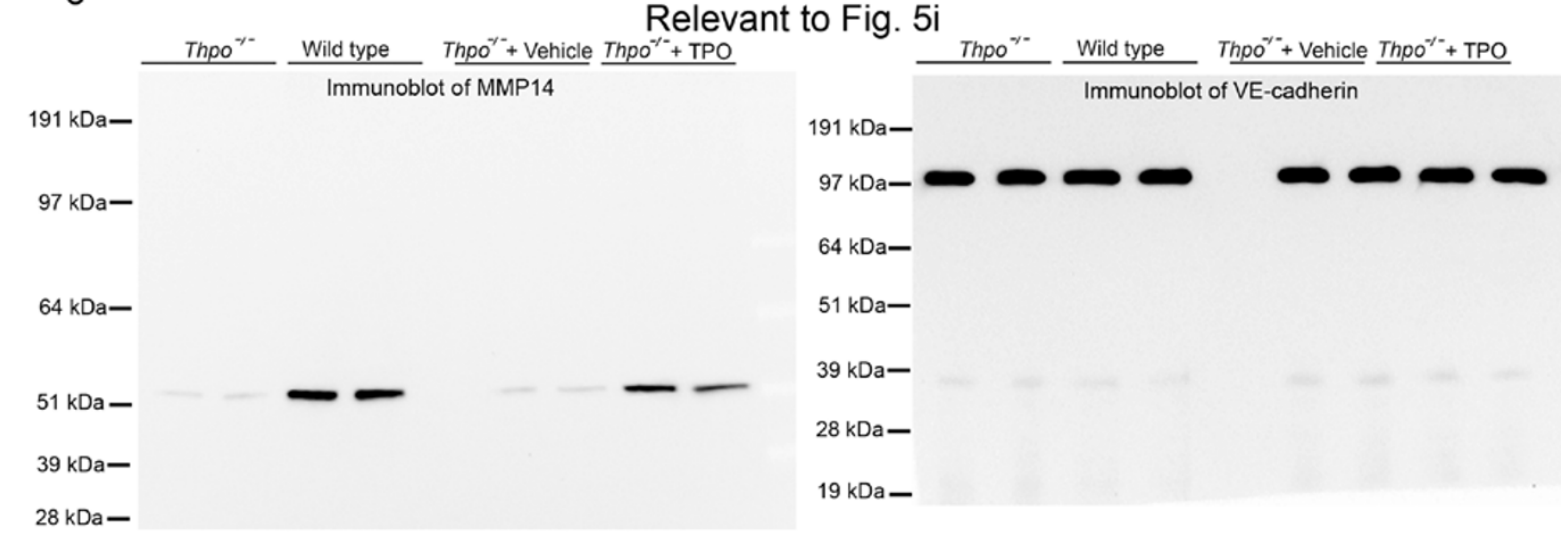

f

$\begin{array}{ll}\underset{\text { PLT: }}{\text { Infused }} & \frac{S d f 1^{+/+}}{191 \mathrm{kDa}-} \\ 97 \mathrm{kDa}- & \end{array}$

$64 \mathrm{kDa}-$

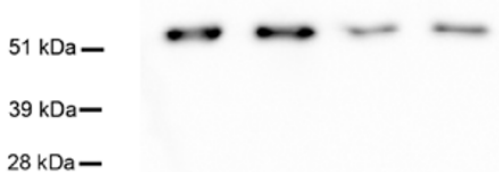

\section{Relevant to Fig. $5 \mathrm{~m}$}

Infused
PLT:
$191 \mathrm{kDa}-$
$97 \mathrm{kDa}-$
$64 \mathrm{kDa}-$
$51 \mathrm{kDa}-$
$39 \mathrm{kDa}-$
$28 \mathrm{kDa}-$

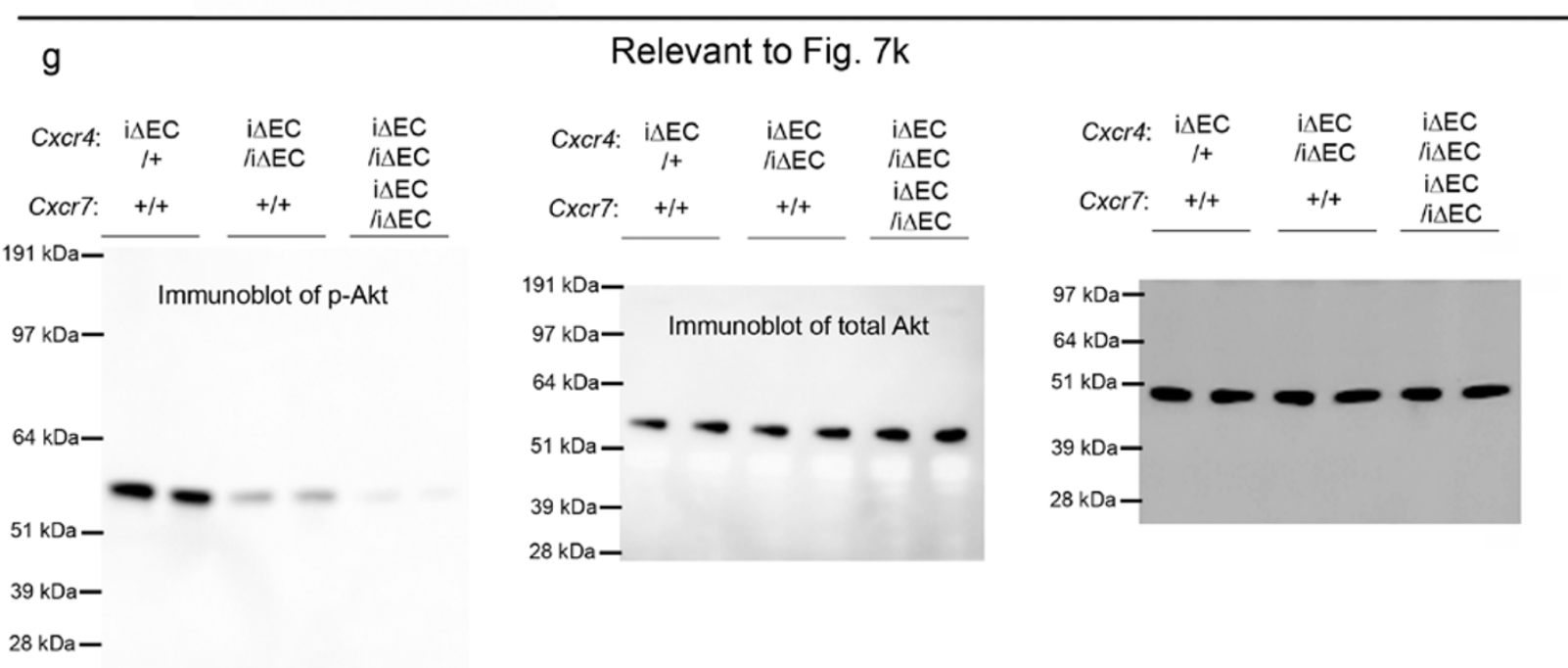




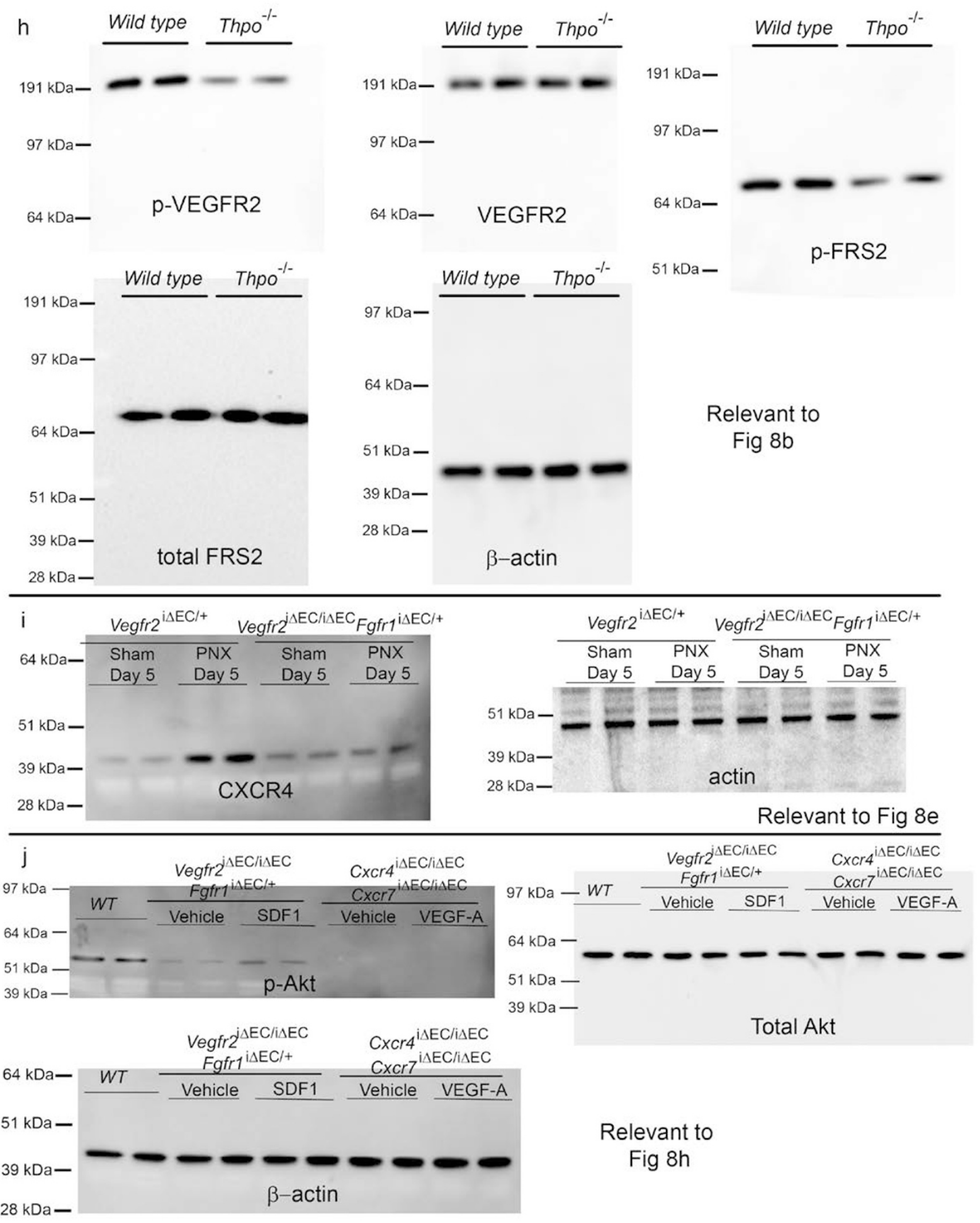

